\title{
Efficient Enantioselective Synthesis of Functionalized Tetrahydropyrans by Ru-Catalyzed Asymmetric Ring-Opening/Cross Metathesis (AROM/CM)
}

\author{
Dennis G. Gillingham, Osamu Kataoka, Steven B. Garber and Amir H. Hoveyda* \\ Department of Chemistry, Merkert Chemistry Center, Boston College \\ Chestnut Hill, Massachusetts 02467
}

\section{SUPPORTING INFORMATION}

General. Infrared (IR) spectra were recorded on a Nicolet 210 spectrophotometer, $\square_{\max }$ in $\mathrm{cm}^{-1}$. Bands are characterized as broad (br), strong (s), medium (m), and weak (w). ${ }^{1} \mathrm{H}$ NMR spectra were recorded on a Varian Unity INOVA $400(400 \mathrm{MHz})$ spectrometer. Chemical shifts are reported in ppm from tetramethylsilane with the solvent resonance as the internal standard $\left(\mathrm{CDCl}_{3}\right.$ : $\left.\square 7.26 \mathrm{ppm}, \mathrm{C}_{6} \mathrm{D}_{6}: \square 7.35 \mathrm{ppm}\right)$. Data are reported as follows: chemical shift, multiplicity $(\mathrm{s}=$ singlet, $\mathrm{d}=$ doublet, $\mathrm{t}=$ triplet, $\mathrm{q}$ $=$ quartet, $\mathrm{br}=$ broad, $\mathrm{m}=$ multiplet $)$, coupling constants $(\mathrm{Hz})$, integration, and assignment. ${ }^{13} \mathrm{C}$ NMR spectra were recorded on a Varian Unity INOVA $400(100 \mathrm{MHz})$ spectrometer with complete proton decoupling. Chemical shifts are reported in ppm from tetramethylsilane with the solvent as the internal reference $\left(\mathrm{CDCl}_{3}: \square 77.16 \mathrm{ppm}\right) .{ }^{19} \mathrm{~F}$ NMR spectra were recorded on a Varian Unity INOVA $400(376 \mathrm{MHz})$ spectrometer with complete proton decoupling. Chemical shifts are reported in ppm from $\mathrm{CFCl}_{3}$ with the solvent as the internal reference. High-resolution mass spectra were recorded at the University of Illinois (Urbana-Champaign, IL) or on a Micromass LCT ESI-MS (positive mode) at the Mass Spectrometry Facility, Boston College. Elemental microanalyses were performed by Robertson Microlit Laboratories (Madison, NJ). Enantiomer ratios were determined by chiral HPLC analysis (Chiral Technologies Chiralpak AS, Chiralpak AD, Chiralcel OJ, Chiralcel OB-H, and Chiralcel OD $(0.46 \mathrm{~cm}$ x $25 \mathrm{~cm}))$ in comparison with authentic racemic materials, or by ${ }^{1} \mathrm{H}$ or ${ }^{19} \mathrm{~F}$ NMR spectroscopy of the derived Mosher esters in comparison with Mosher esters derived from the authentic racemic materials. Optical rotation values were measured on a Rudolph Research Analytical Autopol IV Polarimeter.

Materials. Unless otherwise noted, all reactions were carried out with distilled and degassed solvents under an atmosphere of dry $\mathrm{N}_{2}$ in oven- $\left(135^{\circ} \mathrm{C}\right)$ and flame-dried 
glassware with standard drybox or vacuum-line techniques, and all work-up and purification procedures were carried out with reagent solvents in air. In most instances, solid organometallic compounds were stored under an atmosphere of $\mathrm{N}_{2}$; although it has been determined that such precautions are unnecessary to maintain catalyst stability. All reagent solvents were purchased from Doe and Ingalls, unless otherwise noted. Solvents were purified under positive pressure of dry Ar by a modified Innovative Technologies purification system: toluene and benzene were purified through a copper oxide and an alumina column; $\mathrm{CH}_{2} \mathrm{Cl}_{2}$ and $\mathrm{Et}_{2} \mathrm{O}$ were purged with $\mathrm{Ar}$ and purified by passing them through two alumina columns. THF was purified by distillation from a benzophenone ketyl immediately prior to use. 1,2-Dichloroethane (Lancaster), $\mathrm{CDCl}_{3}$ (Cambridge Isotope Labarotory, Inc.), triethylamine (Acros), styrene (Aldrich), vinylcyclohexane (Aldrich), p-methoxystyrene (Aldrich), p-bromostyrene (Aldrich), and $p$ trifluoromethylstyrene (Aldrich) were distilled from $\mathrm{CaH}_{2}$ under $\mathrm{N}_{2}$. $\mathrm{MeOH}$ was distilled over $\mathrm{Mg}$ under $\mathrm{N}_{2}$. Dimethylformamide (Fisher) was stored under $\mathrm{N}_{2}$ over activated $4 \AA$ molecular sieves. $\mathrm{K}_{2} \mathrm{CO}_{3}$ was stored in an oven $\left(120{ }^{\circ} \mathrm{C}\right) . \mathrm{Cl}_{2} \mathrm{Ru}(=\mathrm{CH}-o$ $\left.\mathrm{O} i \mathrm{PrC}_{6} \mathrm{H}_{3} \mathrm{Ph}\right) \mathrm{PCy}$ was prepared as previously described. ${ }^{1} \mathrm{Cl}_{2} \mathrm{Ru}\left(=\mathrm{CHC}_{6} \mathrm{H}_{5}\right)\left(\mathrm{PCy}_{3}\right)_{2}$ was purchased from Materia. The following materials were purchased from commercial sources and used as received: $\mathrm{EtOH}$ (Fisher), Calcium granules (Aldrich), $\mathrm{Ag}_{2} \mathrm{O}$ (Strem), $0.05 \mathrm{M}$ potassium phosphate monobasic/ sodium hydroxide $\mathrm{pH} 7$ buffer (Fisher), NaI (Aldrich).

Silica gel column chromatography was driven with compressed air and performed with silica gel 60 (230-400 mesh; pH (10\% suspension) 6.5-7.0; surface area $500 \mathrm{~m}^{2} / \mathrm{g}$; pore volume $0.75 \mathrm{ml} / \mathrm{g}$ ) obtained from TSI Chemical Co. (Cambridge, MA).

\section{Preparation of iodide catalyst (1b).}

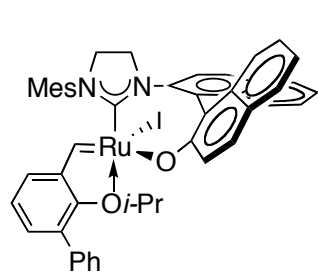

Chloride catalyst 1a $(50.3 \mathrm{mg}, 0.0616 \mathrm{mmol})$ and $\mathrm{NaI}(97.1 \mathrm{mg}$, $0.648 \mathrm{mmol})$ were weighed into a $3 \mathrm{~mL}$ Teflon cap vial, THF (1 $\mathrm{mL}$ ) was added by syringe and the vial was capped and sealed with Teflon tape and electrical tape. The mixture was submerged into a $70{ }^{\circ} \mathrm{C}$ oil bath and stirred for $1 \mathrm{~h}$. The THF was removed with a stream of $\mathrm{N}_{2}$ and the mixture was loaded directly onto a column of silica gel and eluted (1:1 Hex: $\left.\mathrm{CH}_{2} \mathrm{Cl}_{2}\right)$ to deliver $\mathbf{1 b}(47.0 \mathrm{mg}, 85 \%)$ as a brown solid. X-ray quality crystals were obtained by silica gel chromatography in hexane and diethyl ether (9:1); slow

(1) Van Veldhuizen, J. V.; Gillingham, D. G.; Garber, S. B.; Kataoka, O.; Hoveyda, A. H. J. Am. Chem. Soc. 2003, 125, 12502-12508. 
evaporatation of the coloured fractions deliver large crystals after 4-5 days. IR (neat): 3055 (w), 2974 (w), 2917 (w), 1680 (w), 1577 (m), 1470 (m), 1457 (s), 1420 (s), 1281 (s), 910 (m), 734 (s). ${ }^{1} \mathrm{H}$ NMR (400 MHz, $\mathrm{CDCl}_{3}$ ): $\square 15.57$ (s, 1H), 8.14 (dd, $J=8.7,8.7$ $\mathrm{Hz}, 2 \mathrm{H}), 7.93(\mathrm{~d}, J=8.2 \mathrm{~Hz}, 1 \mathrm{H}), 7.75(\mathrm{~d}, J=8.0 \mathrm{~Hz}, 1 \mathrm{H}), 7.63(\mathrm{~d}, J=9.0 \mathrm{~Hz}, 1 \mathrm{H}), 7.43$ (m, 3H), 7.27-7.07 (m, 9H), $6.99(\mathrm{~s}, 1 \mathrm{H}), 6.93$ (ddd, $J=8.0,0.9,0.9 \mathrm{~Hz}, 1 \mathrm{H}), 6.85-6.83$ (m, 2H), 6.79 (d, $J=9.0 \mathrm{~Hz}, 1 \mathrm{H}), 4.28-4.18(\mathrm{~m}, 2 \mathrm{H}), 3.93-3.86(\mathrm{~m}, 1 \mathrm{H}), 3.65-3.58(\mathrm{~m}$, $1 \mathrm{H}), 3.55-3.47(\mathrm{~m}, 1 \mathrm{H}), 2.44(\mathrm{~s}, 3 \mathrm{H}), 2.29(\mathrm{~s}, 3 \mathrm{H}), 1.82(\mathrm{~s}, 3 \mathrm{H}), 0.56(\mathrm{~d}, J=6.3 \mathrm{~Hz}, 3 \mathrm{H})$, $0.33(\mathrm{~d}, J=6.3 \mathrm{~Hz}, 3 \mathrm{H}) .{ }^{13} \mathrm{C} \mathrm{NMR}\left(100 \mathrm{MHz}, \mathrm{CDCl}_{3}\right): \square$ 279.09, 215.03, 169.08, $149.95,148.20$, 139.84, 138.82, 138.33, 138.10, 137.93, 137.58, 137.56, 134.88, 134.74, $133.69,132.12$, 131.61, 130.03, 129.56, 129.30, 128.86, 128.80, 128.64, 128.32, 128.23, $128.19,127.93$, 127.76, 126.74, 126.54, 126.09, 125.18, 124.79, 124.07, 121.65, 120.81, $119.24,78.29,53.32,51.81,21.49,20.27,20.24,20.10,18.64$. X-ray data is included at the end of this document.

General procedure A: Ru-Catalyzed AROM/CM in THF at 0.1 M. 11a $(12.3 \mathrm{mg}$, $98.0 \square \mathrm{mol})$ and styrene $(46.4 \mathrm{mg}, 0.446 \mathrm{mmol})$ were combined in a dried $3 \mathrm{~mL}$ vial containing a stir bar under nitrogen. Catalyst $\mathbf{1 b}(3.6 \mathrm{mg}, 4.4 \square \mathrm{mol})$ was dissolved in THF $(1 \mathrm{~mL})$ and added by cannula to the vial containing substrate and styrene. The reaction was allowed to stir at $22{ }^{\circ} \mathrm{C}$ for $3 \mathrm{~h}$ at which point the solvent was removed with a stream of $\mathrm{N}_{2}$ and the mixture was loaded onto a silica gel column $(0.5 \mathrm{~cm} \mathrm{~W} \times 8 \mathrm{~cm} \mathrm{~L})$ and eluted (3:2 Hex:Et $\left.\mathrm{E}_{2} \mathrm{O}\right)$ to deliver recovered catalyst $\left(1.7 \mathrm{mg}, \mathrm{R}_{f}=0.5,51 \%\right)$ and the desired product $12\left(14.1 \mathrm{mg}, \mathrm{R}_{f}=0.2,64 \%\right)$ as a clear colourless oil.

\section{General procedure B: Ru-Catalyzed AROM/CM in the absence of solvent.} Compound 5c $(10.0 \mathrm{mg}, 46.0 \square \mathrm{mol})$ and distilled vinylcyclohexane (67.2 mg, 0.610 mmol) were combined in a dried $3 \mathrm{~mL}$ vial in the glovebox and allowed to stir until all of 5c dissolved. Catalyst 3 ( $2.2 \mathrm{mg}, 2.4 \square \mathrm{mol})$ was then weighed directly into the vial. The resulting solution was stirred for $2 \mathrm{~h}$ at $22{ }^{\circ} \mathrm{C}$ at which point TLC analysis (4:1 Hex:Et $\left.2 \mathrm{O}\right)$ indicated complete consumption of starting material. The crude mixture was loaded directly onto a silica gel column $(0.5 \mathrm{~cm} \mathrm{~W} \times 8 \mathrm{~cm} \mathrm{~L})$ and eluted $\left(9: 1 \mathrm{Hex}_{\mathrm{Et}} \mathrm{O}\right)$ to deliver bis-cross product $\left(1.4 \mathrm{mg}, \mathrm{R}_{f}=0.7,10 \%\right)$, desired product $16\left(9.1 \mathrm{mg}, \mathrm{R}_{f}=0.5\right.$, $61 \%)$ and recovered catalyst $\left(2.0 \mathrm{mg}, \mathrm{R}_{f}=0.3,90 \%\right)$.

General procedure C: Ru-Catalyzed AROM/CM by slow addition of olefin partner. Catalyst 1a $(3.7 \mathrm{mg}, 5.0 \square \mathrm{mol})$ and vinylcyclohexane $(48.0 \mathrm{mg}, 0.436 \mathrm{mmol})$ were weighed into a dried $3 \mathrm{~mL}$ vial containing a stir bar. Substrate $\mathbf{2 a}(11.0 \mathrm{mg}, 87.0 \square \mathrm{mol})$ 
was added slowly by syringe or syringe pump as a solution in dichloroethane over $1 \mathrm{~h}$ at $22{ }^{\circ} \mathrm{C}$. The reaction was allowed to stir for an additional $12 \mathrm{~h}$ at $22{ }^{\circ} \mathrm{C}$ at which time the solvent was removed under a stream of $\mathrm{N}_{2}$ and the mixture was loaded directly onto a silica gel column $(0.5 \mathrm{~cm} \mathrm{~W} \times 8 \mathrm{~cm} \mathrm{~L})$ and eluted $\left(2: 1 \mathrm{Hex}_{\mathrm{Et}} \mathrm{O}\right)$ to deliver product 9 $\left(14.8 \mathrm{mg}, \mathrm{R}_{f}=0.3,72 \%\right)$ as a clear colourless oil.

\section{(2S,4R,6R)-Tetrahydro-2-(E)-styryl-6-vinyl-2H-pyran-4-ol (4a).}

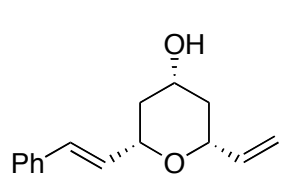

General procedure A was followed with substrate $\mathbf{2 a}$ to afford a colourless oil after silica gel chromatography $\left(3: 1 \mathrm{Hex}_{\mathrm{Et}} \mathrm{O}, \mathrm{R}_{f}=\right.$ 0.15). IR (neat): 3370 (br), 3074 (w), 3018 (w), 2955 (m), 2898 (m), 2848 (m), 1646 (m), 1488 (w), 1451 (w), 1362 (m), 1306 (m), 1054

(s), 960 (s), 758 (s), 695 (s). ${ }^{1} \mathrm{H}$ NMR (400 MHz, $\mathrm{CDCl}_{3}$ ): $\square 7.38-7.19$ (m, 5H), 6.62 (d, $J=16.0 \mathrm{~Hz}, 1 \mathrm{H}), 6.24(\mathrm{dd}, J=$ $16.0,6.0 \mathrm{~Hz}, 1 \mathrm{H}), 5.91$ (ddd, $J=$ $16.0,10.8,6.0 \mathrm{~Hz}, 1 \mathrm{H}), 5.30(\mathrm{dd}, J$ $=17.6,1.2 \mathrm{~Hz}, 1 \mathrm{H}), 5.15(\mathrm{dd}, J=$ $10.8,1.2 \mathrm{~Hz}, 1 \mathrm{H}), 4.09-4.05(\mathrm{~m}$, $1 \mathrm{H}), 3.97-3.90(\mathrm{~m}, 2 \mathrm{H}), 2.12-2.04$ $(\mathrm{m}, 2 \mathrm{H}), 1.55$ (br s, 1H), 1.44-1.27 $(\mathrm{m}, 2 \mathrm{H}) .{ }^{13} \mathrm{C}$ NMR $(100 \mathrm{MHz}$,

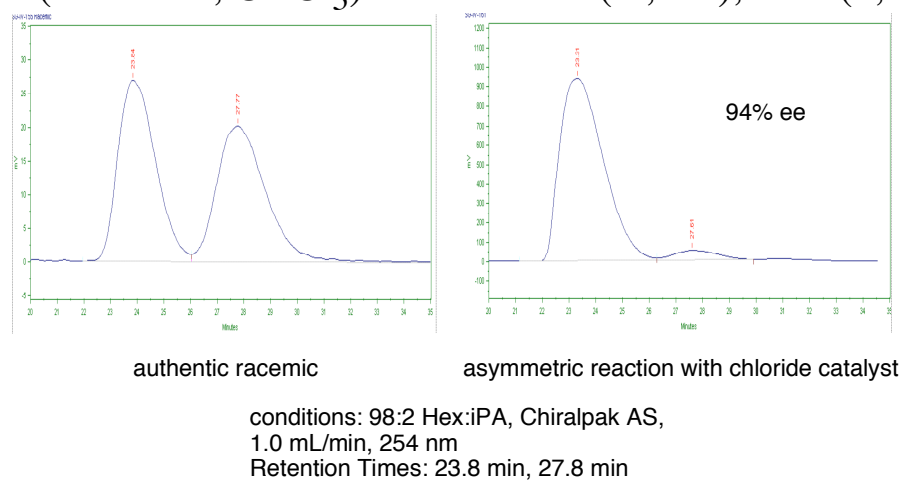
$\mathrm{CDCl}_{3}$ ): $\square 138.29,136.94,130.84,129.59,128.72,127.86,126.72,115.77,76.45,76.19$, 68.28, 41.31, 40.89. HRMS Calcd for $\mathrm{C}_{15} \mathrm{H}_{18} \mathrm{O}_{2} \mathrm{Na}: 253.1204$. Found: 253.1202 . Absolute configuration is determined by conversion to 28 (See S18 \& S19 for details).

$(2 S, 4 R, 6 R)-4-B e n z y l o x y t e t r a h y d r o-2-(E)$-styryl-6-vinyl-2H-pyran (4b).

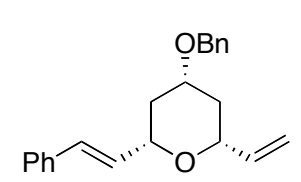

General procedure A was followed with substrate $\mathbf{2 b}$ to afford a colourless oil after silica gel chromatography (40:1 Hex:Et $2 \mathrm{O}, \mathrm{R}_{f}=$ 0.15). IR (neat): 2922 (m), 2853 (m), 1495 (w), 1451 (w), 1354 (m), $1157(\mathrm{w})$,

$1071(\mathrm{~s}), 966$ (m), 747 (s), 695

(s). $\quad{ }^{1} \mathrm{H} \quad \mathrm{NMR}(400 \mathrm{MHz}$, $\left.\mathrm{CDCl}_{3}\right)$ : $\square 7.42-7.20(\mathrm{~m}, 10 \mathrm{H})$, $6.63(\mathrm{~d}, J=16.9 \mathrm{~Hz}, 1 \mathrm{H}), 6.26$ (dd, $J=16.1,6.0 \mathrm{~Hz}, 1 \mathrm{H}), 5.95$ (ddd, $J=17.2,10.6,5.7 \mathrm{~Hz}$, 1H), 5.32 (ddd, $J=17.2,1.5,1.5$

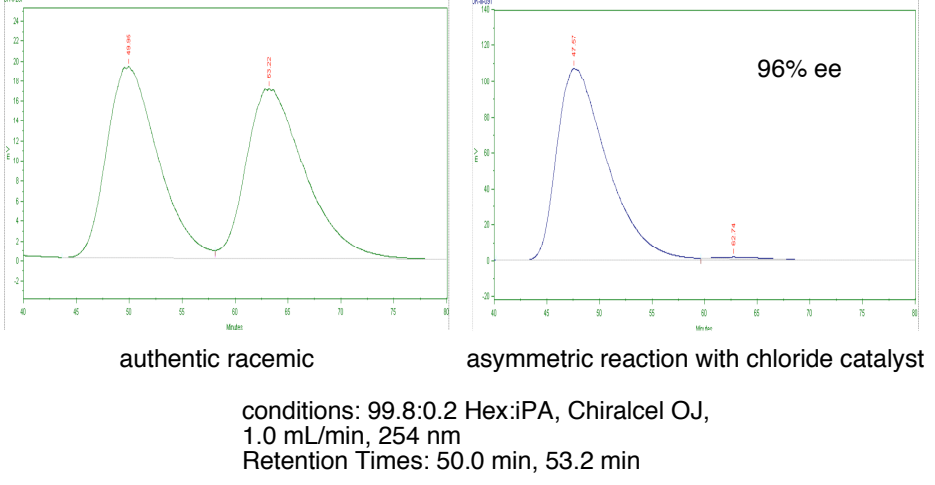


$\mathrm{Hz}, 1 \mathrm{H}), 5.17$ (ddd, $J=10.6,1.5,1.5 \mathrm{~Hz}, 1 \mathrm{H}), 4.62$ (s, 2H), 4.10-4.03 (m, 1H), 3.98-3.91 (m, 1H), 3.71 (dddd, $J=11.0,11.0,4.6,4.6 \mathrm{~Hz}, 1 \mathrm{H}), 2.21$ (dddd, $J=12.5,4.4,2.2,2.2$ $\mathrm{Hz}, 1 \mathrm{H}), 2.15$ (dddd, $J=12.5,4.4,2.2,2.2 \mathrm{~Hz}, 1 \mathrm{H}), 1.48$ (ddd, $J=11.4,11.4,11.4 \mathrm{~Hz}$, $1 \mathrm{H}), 1.42(\mathrm{ddd}, J=11.4,11.4,11.4 \mathrm{~Hz}, 1 \mathrm{H}) .{ }^{13} \mathrm{C} \mathrm{NMR}\left(100 \mathrm{MHz}, \mathrm{CDCl}_{3}\right): \square$ 138.66, $138.39,136.91,130.74,129.70,128.65,128.60,127.77,127.72,126.66,115.65,76.53$, 76.27, 74.54, 69.79, 38.21, 37.83. HRMS Calcd for $\mathrm{C}_{22} \mathrm{H}_{24} \mathrm{O}_{2}$ : 320.1776. Found: 320.1767. Absolute configuration is determined by conversion to $\mathbf{4 a}$.

\section{Tetrahydro-4-methoxy-2-(E)-styryl-6-vinyl-2H-pyran (4c).}

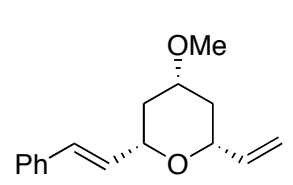

General procedure A was followed with substrate $2 \mathbf{c}$ to afford a colourless oil after silica gel chromatography $\left(20: 1 \mathrm{Hex}_{\mathrm{Et}} \mathrm{O}, \mathrm{R}_{f}=\right.$ 0.14). IR (neat): 2945 (m), 2923 (m), 2848 (m), 2824 (m), 1448 (m), 1379 (m), 1309 (m), 1081 (s), 966 (m), 748 (m), 694 (m). ${ }^{1} \mathrm{H}$ NMR (400 $\left.\mathrm{MHz}, \mathrm{CDCl}_{3}\right): \square 7.42-7.20(\mathrm{~m}$, $5 \mathrm{H}), 6.63(\mathrm{~d}, J=15.4 \mathrm{~Hz}, 1 \mathrm{H}), 6.26$ $(\mathrm{dd}, J=15.9,6.0 \mathrm{~Hz}, 1 \mathrm{H}), 5.94$ (ddd, $J=17.4,10.6,5.7 \mathrm{~Hz}, 1 \mathrm{H}$ ), 5.32 (ddd, $J=17.4,1.5,1.5 \mathrm{~Hz}, 1 \mathrm{H})$, 5.17 (ddd, $J=10.4,1.5,1.5 \mathrm{~Hz}, 1 \mathrm{H})$, 4.08 (dddd, $J=11.2,6.1,1.5,1.5$ $\mathrm{Hz}, 1 \mathrm{H}), 4.00-3.81(\mathrm{~m}, 1 \mathrm{H}), 3.50$ (dddd, $J=11.0,11.0,4.4,4.4 \mathrm{~Hz}$,

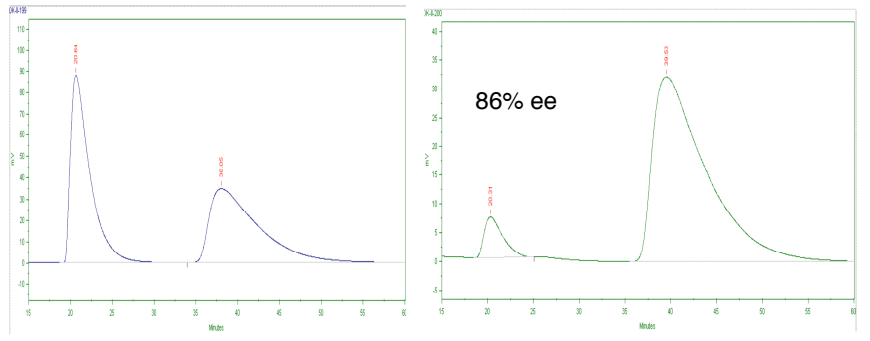

authentic racemic asymmetric reaction with chloride catalyst conditions: 99.7:0.3 Hex:iPA, Chiralpak AD, $0.1 \mathrm{~mL} / \mathrm{min}, 254 \mathrm{~nm}$ Retention Times: $20.6 \mathrm{~min}, 36.1 \mathrm{~min}$

1H), 3.40 (s, 3H), 2.17 (dddd, $J=12.5,4.4,2.2,2.2 \mathrm{~Hz}, 1 \mathrm{H}), 2.12$ (dddd, $J=12.5,4.4$, 2.2, $2.2 \mathrm{~Hz}, 1 \mathrm{H}), 1.36$ (ddd, $J=11.4,11.4,11.4 \mathrm{~Hz}, 1 \mathrm{H}), 1.30$ (ddd, $J=11.4,11.4,11.4$ $\mathrm{Hz}, 1 \mathrm{H}) .{ }^{13} \mathrm{C} \mathrm{NMR}\left(100 \mathrm{MHz}, \mathrm{CDCl}_{3}\right): \square 138.38,136.88,130.72,129.69,126.65$, 127.77, 126.65, 115.66, 76.60, 76.51, 76.26, 55.57, 37.80, 37.41. HRMS Calcd for $\mathrm{C}_{16} \mathrm{H}_{20} \mathrm{O}_{2}$ : 244.1463. Found: 244.1470.

\section{$(2 S, 4 R, 6 R)-4-[($ tert-Butyldimethylsilyl)oxy]tetrahydro-2-styryl-6-vinyl-2H-pyran}

(4d).

General procedure A was followed with substrate $2 d$ to afford a
colourless oil after silica gel chromatography $\left(50: 1 \operatorname{Hex}_{2} \mathrm{Et}_{2} \mathrm{O}, \mathrm{R}_{f}=\right.$ 1255 (m), 1075 (s), 914 (m), 837 (m), 776 (m), 746 (m), 693 (m). ${ }^{1} \mathrm{H}$ NMR (400 MHz, $\left.\mathrm{CDCl}_{3}\right): \square 7.44-7.16(\mathrm{~m}, 5 \mathrm{H}), 6.61(\mathrm{~d}, J=15.9 \mathrm{~Hz}, 1 \mathrm{H}), 6.24(\mathrm{dd}, J=15.9,6.1 \mathrm{~Hz}, 1 \mathrm{H})$, $5.92(\mathrm{ddd}, J=17.2,10.4,6.0 \mathrm{~Hz}, 1 \mathrm{H}), 5.31(\mathrm{ddd}, J=17.2,1.5,1.5 \mathrm{~Hz}, 1 \mathrm{H}), 5.15(\mathrm{~d}, J=$ 
$10.4 \mathrm{~Hz}), 4.12-4.01(\mathrm{~m}, 1 \mathrm{H})$, 3.99-3.83 (m, 2H), 2.00-1.88 (m, 2H), 1.45 (ddd, $J=11.9,11.9,11.9$ Hz, 1H), 1.39 (ddd, $J=11.9,11.9$, $11.9 \mathrm{~Hz}, 1 \mathrm{H}), 0.90$ (s, 9H), 0.09 (s, 6H). ${ }^{13} \mathrm{C}$ NMR $(100 \mathrm{MHz}$,

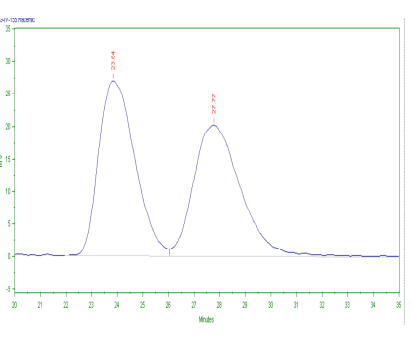

authentic racemic

$\left.\mathrm{CDCl}_{3}\right): \square 138.51,136.96,130.62$, $129.85,128.63,127.71,126.64$, 115.57, 76.58, 76.30, 68.77, 41.73, 41.32, 25.97, 18.26, -4.35. HRMS

conditions: 98:2 Hex:iPA, Chiralpak AS, $1.0 \mathrm{~mL} / \mathrm{min}, 254 \mathrm{~nm}$

Retention Times: $23.8 \mathrm{~min}, 27.8 \mathrm{~min}$

Calcd for $\mathrm{C}_{21} \mathrm{H}_{32} \mathrm{O}_{2} \mathrm{Si}$ : 344.2172 .

Found: 344.2167.

Enantiopuritiy of $\mathbf{4 d}$ was

determined by analysis of HPLC of the corresponding alcohol after desilylation. Absolute configuration is determined by conversion to $\mathbf{4 a}$.

\section{Tetrahydro-2-[(E)-2-(4-methoxyphenyl)vinyl]-6-vinyl-2H-pyran-4-ol (5).}

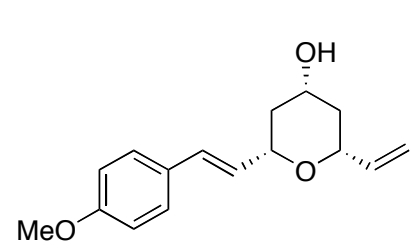

General procedure A was followed with substrate 1a to afford a colorless oil after silica gel chromatography $\left(3: 1 \mathrm{Hex}_{\mathrm{Et}} \mathrm{O}\right.$, $\left.\mathrm{R}_{f}=0.15\right)$. IR (neat): $3388(\mathrm{br}), 2939(\mathrm{~m}), 2920(\mathrm{~m}), 1607(\mathrm{~s})$, $1512(\mathrm{~s}), 1301(\mathrm{~m}), 1248(\mathrm{~s}), 1175(\mathrm{~m}), 1063(\mathrm{~m}), 1035(\mathrm{~m})$, 969 (m), 845 (m). ${ }^{1} \mathrm{H}$ NMR (400 MHz, $\left.\mathrm{CDCl}_{3}\right): \square 7.31$ (d, $J=$

$8.8 \mathrm{~Hz}, 2 \mathrm{H}), 6.84(\mathrm{~d}, J=8.8 \mathrm{~Hz}, 2 \mathrm{H}), 6.56(\mathrm{~d}, J=15.9 \mathrm{~Hz}, 1 \mathrm{H}), 6.11(\mathrm{dd}, J=15.9,6.2$ $\mathrm{Hz}, 1 \mathrm{H}), 5.92$ (ddd, $J=17.4$, $10.6,6.0 \mathrm{~Hz}, 1 \mathrm{H}), 5.31$ (ddd, $J=$ $17.4,1.1,1.1 \mathrm{~Hz}, 1 \mathrm{H}), 5.16$ (ddd, $J=10.6,1.1,1.1 \mathrm{~Hz}, 1 \mathrm{H}), 4.05$ (dddd, $J=11.4,6.2,1.1,1.1 \mathrm{~Hz}$, $1 \mathrm{H}), 3.99-3.87$ (m, 2H), 3.80 (s, $3 \mathrm{H}), 2.15-2.00(\mathrm{~m}, 2 \mathrm{H}), 1.71$ (br s, $1 \mathrm{H}), 1.41(\mathrm{dd}, J=11.4,11.4$ $\mathrm{Hz}, 1 \mathrm{H}), 1.32$ (dd, $J=11.4,11.4$ $\mathrm{Hz}, 1 \mathrm{H}) .{ }^{13} \mathrm{C}$ NMR $(100 \mathrm{MHz}$, $\left.\mathrm{CDCl}_{3}\right): \square 159.40, \quad 138.27$, conditions: $85: 15$ Hex:iPA, Chiralpak AS, $1.0 \mathrm{~mL} / \mathrm{min}, 254 \mathrm{~nm}$ Retention Times: $15.4 \mathrm{~min}, 29.9 \mathrm{~min}$

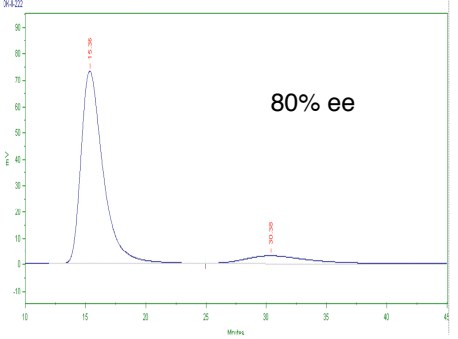

asymmetric reaction with chloride catalyst

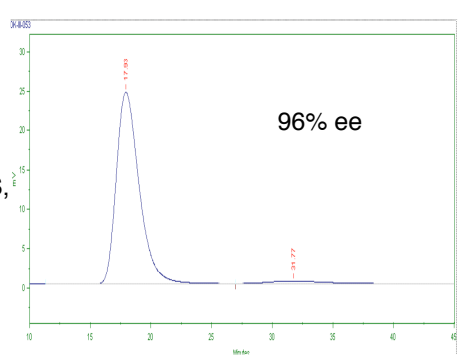

asymmetric reaction with iodide catalyst 
55.40, 41.30, 40.79. HRMS Calcd for $\mathrm{C}_{16} \mathrm{H}_{20} \mathrm{O}_{3} \mathrm{Na}$ : 283.1310. Found: 283.1304.

\section{2-[(E)-4-(Trifluoromethyl)styryl]tetrahydro-6-vinyl-2H-pyran-4-ol (6).}

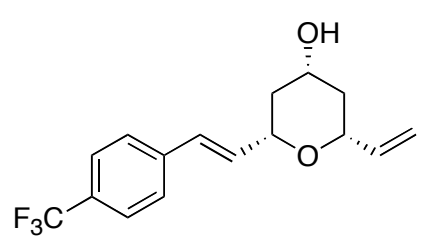

General procedure A was followed with substrate 1a to afford a colorless oil after silica gel chromatography $\left(3: 1 \mathrm{Hex}_{\mathrm{E}} \mathrm{t}_{2} \mathrm{O}\right.$, $\left.\mathrm{R}_{f}=0.18\right)$. IR (neat): $3387(\mathrm{br}), 2943(\mathrm{~m}), 2921$ (m), 1615 (m), 1415 (m), 1325 (s), 1165 (s), 1124 (s), 1067 (s), 970 (m), $858(\mathrm{~m}) .{ }^{1} \mathrm{H}$ NMR $\left(400 \mathrm{MHz}, \mathrm{CDCl}_{3}\right): \square 7.56(\mathrm{~d}, J=8.2 \mathrm{~Hz}$,

2H), $7.47(\mathrm{~d}, J=8.2 \mathrm{~Hz}, 2 \mathrm{H}), 6.67(\mathrm{~d}, J=16.0 \mathrm{~Hz}, 1 \mathrm{H}), 6.33(\mathrm{dd}, J=16.0,5.6 \mathrm{~Hz}, 1 \mathrm{H})$, 5.94 (ddd, $J=17.3,10.6,5.6$ $\mathrm{Hz}, 1 \mathrm{H}), 5.33$ (ddd, $J=17.3$, $1.5,1.5 \mathrm{~Hz}, 1 \mathrm{H}), 5.18$ (ddd, $J=$ $10.6,1.5,1.5 \mathrm{~Hz}, 1 \mathrm{H})$, 4.15-4.07 (m, 1H), 4.01-3.90 (m, 2H), 2.13 (dddd, $J=12.4$, 4.6, 2.2, $2.2 \mathrm{~Hz}, 1 \mathrm{H}), 2.08$ (dddd, $J=12.4,4.6,2.2,2.2 \mathrm{~Hz}$, 1H), 1.58 (br s, 1H), 1.40 (ddd, $J=11.5,11.5,11.5 \mathrm{~Hz}, 1 \mathrm{H})$, 1.36 (ddd, $J=11.5,11.5,11.5$ $\mathrm{Hz}, 1 \mathrm{H}) .{ }^{13} \mathrm{C}$ NMR (100 MHz,

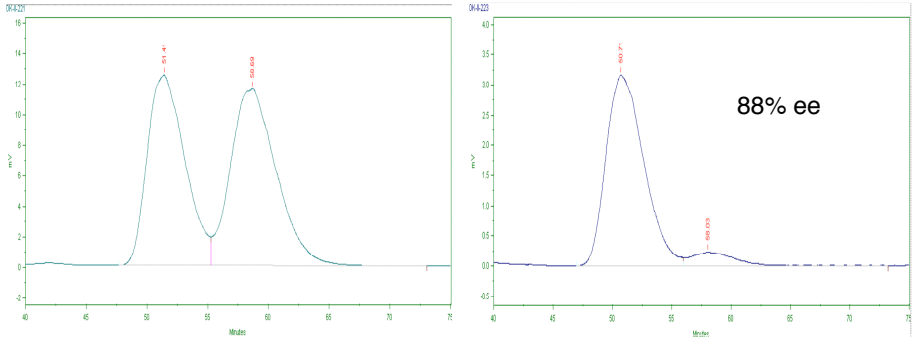

authentic racemic

asymmetric reaction with chloride catalyst Conditions: 99:1 Hex:iPA, Chiralpak AS, $1.0 \mathrm{~mL} / \mathrm{min}, 254 \mathrm{~nm}$ Retention Times: $51.4 \mathrm{~min}, 58.7 \mathrm{~min}$ $\left.\mathrm{CDCl}_{3}\right): \quad$ 140.38, 138.05, $132.18,129.23(\mathrm{q}, J=32.4 \mathrm{~Hz})$,

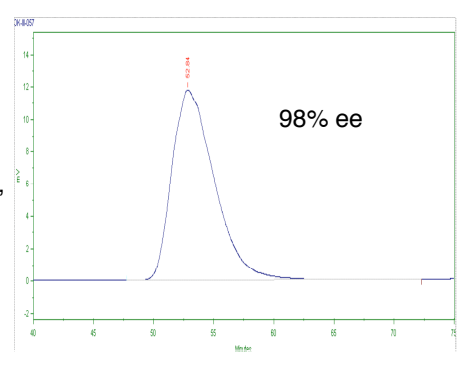

asymmetric reaction with iodide catalyst $129.17,126.77,125.63(\mathrm{q}, J=3.8 \mathrm{~Hz}), 115.85,76.45,75.69,41.05,40.79 .{ }^{19} \mathrm{~F} \mathrm{NMR}$ (376 MHz, $\mathrm{CDCl}_{3}$ ): $\square-63.01$. HRMS Calcd for $\mathrm{C}_{16} \mathrm{H}_{17} \mathrm{~F}_{3} \mathrm{O}_{2}$ : 298.1181. Found: 298.1174.

\section{2-[(E)-2-(4-Bromophenyl)vinyl]tetrahydro-6-vinyl-2H-pyran-4-ol (7).}

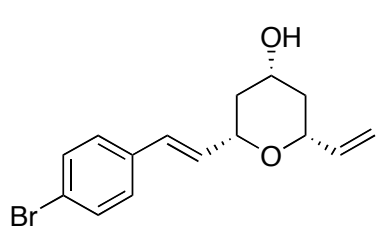

General procedure A was followed with 2 a to afford a colorless solid (mp 85.0-86.0 ${ }^{\circ} \mathrm{C}$, pentane/Et $2 \mathrm{O}$ ) after silica gel chromatography ( $\left.3: 1 \mathrm{Hex}_{\mathrm{Et}} \mathrm{O}, \mathrm{R}_{f}=0.18\right)$. IR (neat): 3365 (br), 2935 (m), 2851 (m), 1487 (s), 1403 (m), 1307 (m), 1064 (s), $1008(\mathrm{~m}), 963(\mathrm{~m}), 808(\mathrm{~m}) .{ }^{1} \mathrm{H}$ NMR (400 MHz, $\left.\mathrm{CDCl}_{3}\right)$ : 7.45-7.39 (m, 2H), 7.31-7.25 (m, 2H), 6.57 (dd, $J=15.9,0.9 \mathrm{~Hz}, 1 \mathrm{H}), 6.23$ (dd, $J=15.9$, $5.9 \mathrm{~Hz}, 1 \mathrm{H}), 5.92$ (ddd, $J=17.4,10.6,5.7 \mathrm{~Hz}, 1 \mathrm{H}), 5.31$ (ddd, $J=17.4,1.5,1.5 \mathrm{~Hz}, 1 \mathrm{H})$, $5.17(\mathrm{ddd}, J=10.6,1.3,1.3 \mathrm{~Hz}, 1 \mathrm{H}), 4.06(\mathrm{dddd}, J=11.2,5.9,1.7,1.7 \mathrm{~Hz}, 1 \mathrm{H})$, 
4.00-3.87 (m, 2H), 2.15-2.01 (m, 2H), 1.67 (br s, 1H), 1.38 (ddd, $J=11.4,11.4,11.4 \mathrm{~Hz}$, $1 \mathrm{H}), 1.34$ (ddd, $J=11.4,11.4,11.4 \mathrm{~Hz}, 1 \mathrm{H}) .{ }^{13} \mathrm{C} \mathrm{NMR}\left(100 \mathrm{MHz}, \mathrm{CDCl}_{3}\right): \square 138.11$, 135.82 , 131.77, 130.30, 129.51, 128.16, 121.55, 115.78, 76.41, 75.87, 68.12, 41.10, 40.79. HRMS Calcd for $\mathrm{C}_{15} \mathrm{H}_{17} \mathrm{BrO}_{2}: 308.0412$. Found: 308.0417.
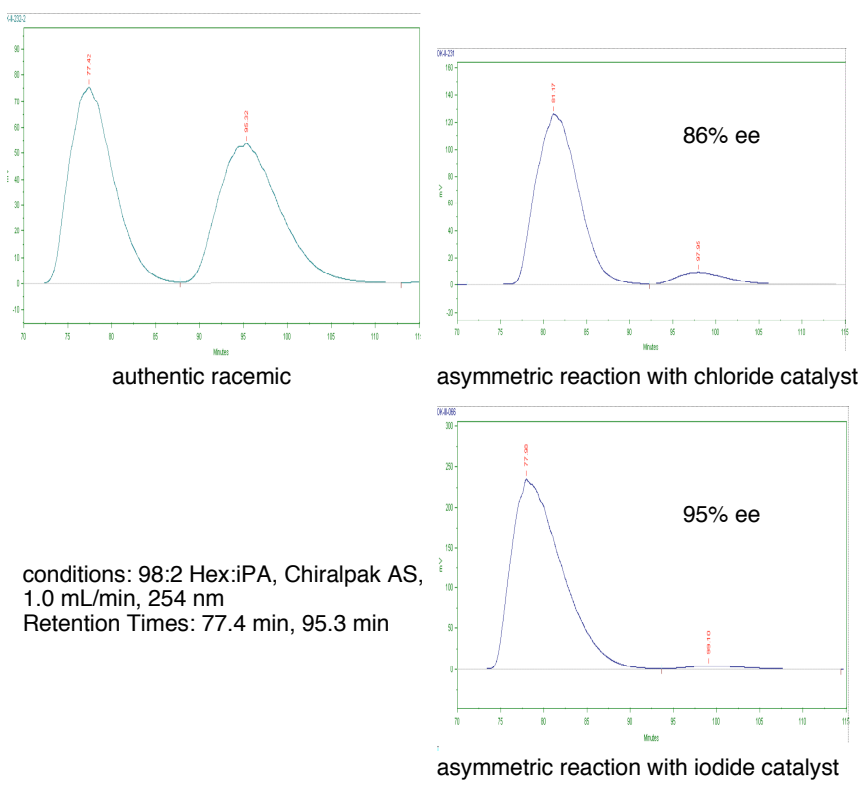

Tetrahydro-2-[(E)-2-(2-methylphenyl)vinyl]-6-vinyl-2H-pyran-4-ol (8).<smiles>C=C[C@H]1C[C@H](O)C[C@@H](/C=C/c2ccccc2[N+](=O)[O-])O1</smiles>

General procedure A was followed with substrate $\mathbf{2 a}$ to afford a colorless oil after silica gel chromatography (3:1 Hex:Et ${ }_{2} \mathrm{O}, \mathrm{R}_{f}=$ 0.18). IR (neat): 3388 (br), 2942 (m), 2920 (m), 2852 (m), 1514 (m), 1359 (m), $1309(\mathrm{~m}), 1063(\mathrm{~m}), 968(\mathrm{~m}), 925(\mathrm{~m}), 796(\mathrm{~m}) .{ }^{1} \mathrm{H}$ NMR (400 MHz, $\mathrm{CDCl}_{3}$ ): ]7.48-7.42 (m, 1H), 7.18-7.10 (m, 3H), 6.84 (dd, $J=15.8$, $1.1 \mathrm{~Hz}, 1 \mathrm{H}), 6.14(\mathrm{dd}, J=15.8,6.0 \mathrm{~Hz}, 1 \mathrm{H}), 5.94(\mathrm{ddd}, J=17.2,10.6,5.5 \mathrm{~Hz}, 1 \mathrm{H}), 5.33$ (ddd, $J=17.2,1.5,1.5 \mathrm{~Hz}, 1 \mathrm{H}), 5.17$ (ddd, $J=10.6,1.5,1.5 \mathrm{~Hz}, 1 \mathrm{H}), 4.10$ (dddd, $J=$ $11.4,6.0,1.7,1.7 \mathrm{~Hz}, 1 \mathrm{H})$, 4.01-3.89 (m, 2H), $2.35(\mathrm{~s}$, $3 \mathrm{H}), 2.12(\mathrm{dddd}, J=12.5,4.8$, 2.2, $2.2 \mathrm{~Hz}, 1 \mathrm{H}$ ), 2.07 (dddd, $J$ $=12.5,4.8,2.2,2.2 \mathrm{~Hz}, 1 \mathrm{H})$, 1.59 (br s, 1H), 1.42 (ddd, $J=$ 11.4, 11.4, $11.4 \mathrm{~Hz}, 1 \mathrm{H}), 1.35$ (ddd, $J=11.4,11.4,11.4 \mathrm{~Hz}$, 1H). ${ }^{13} \mathrm{C}$ NMR (100 MHz, $\left.\mathrm{CDCl}_{3}\right): \square 138.40,136.03$, $135.80,130.99,130.48$, 128.64, 127.77, 126.31, 125.93, 115.67, 77.47, 76.43,

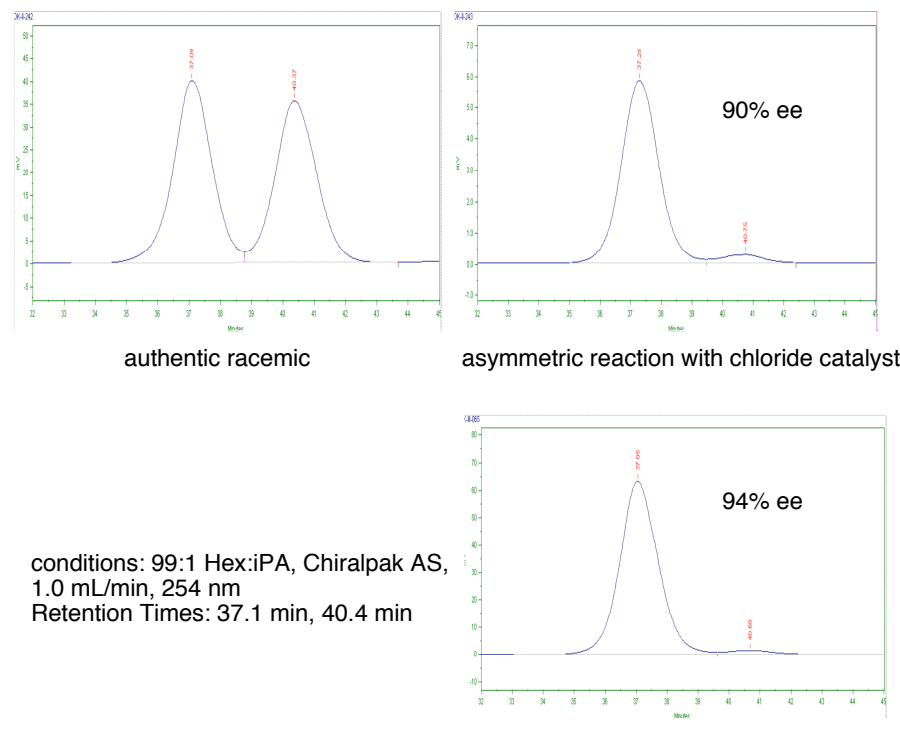

asymmetric reaction with iodide catalyst 
68.35, 41.50, 40.97, 20.02. HRMS Calcd for $\mathrm{C}_{16} \mathrm{H}_{20} \mathrm{O}_{2}$ : 244.1463. Found: 244.1467.

\section{2-[(E)-2-Cyclohexylvinyl]tetrahydro-6-vinyl-2H-pyran-4-ol (9).}

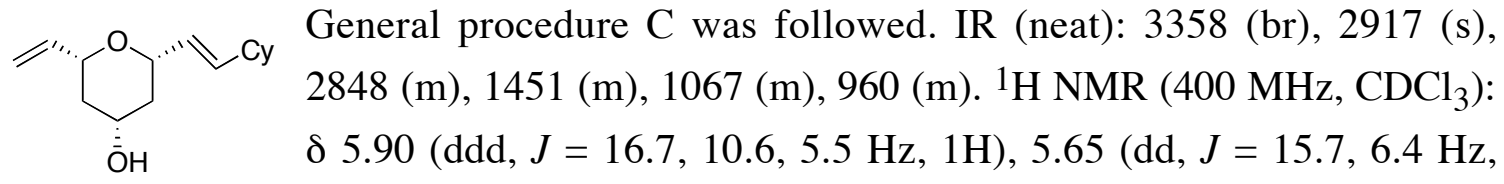
$1 \mathrm{H}), 5.46(\mathrm{dd}, J=15.7,6.2 \mathrm{~Hz}, 1 \mathrm{H}), 5.27(\mathrm{~d}, J=16.7 \mathrm{~Hz}, 1 \mathrm{H}), 5.13(\mathrm{~d}, J=10.6 \mathrm{~Hz}, 1 \mathrm{H})$, 3.90-3.83 (m, 3H), 2.03-1.95 (m, 3H), 1.72-1.62 (m, 5H), 1.30-1.01 (m, 8H). ${ }^{13} \mathrm{C}$ NMR (100 MHz, $\left.\mathrm{CDCl}_{3}\right)$ : $\square 138.44,138.27,127.61,115.53,76.45,76.28,68.24,41.45,40.80$, $40.41,32.79,26.31$, 26.17. HRMS Calcd for $\mathrm{C}_{15} \mathrm{H}_{24} \mathrm{O}_{2} \mathrm{Na}$ : 259.1674. Found: 259.1676. The ee for this compound was determined by analysis of the ${ }^{1} \mathrm{H}$ NMR of the derived Mosher ester (using (S)-Mosher's acid).
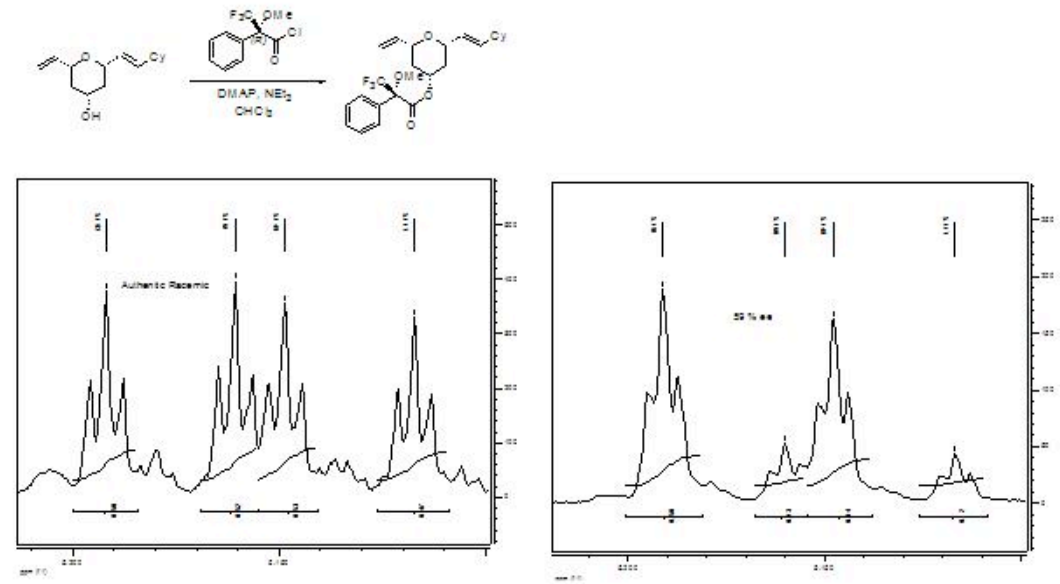

$1 \mathrm{H}$ NMR in $\mathrm{C}_{6} \mathrm{D}_{6}$ analysis of Mosher Ester derived from endo-cyclohexyl ROCM

\section{(2S,4S,6R)-Tetrahydro-2-(E)-styryl-6-vinyl-2H-pyran-4-ol (12).}

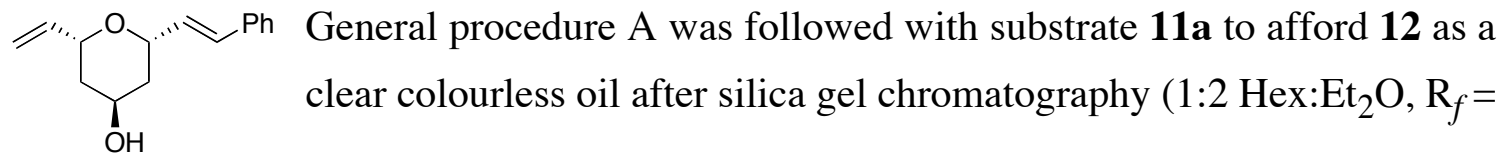

0.3). IR (neat): 3415 (br), 3024

(w), $2917(\mathrm{~m}), 1430(\mathrm{w}), 1300$

(m), $1055(\mathrm{~s}), 960(\mathrm{~s}), 746(\mathrm{~s})$, 696 (s). ${ }^{1} \mathrm{H}$ NMR (400 MHz, $\left.\mathrm{CDCl}_{3}\right):$ 7.39-7.37 (m, 2H), 7.32-7.28 (m, 2H), 7.24-7.20 (m, $1 \mathrm{H}), 6.63(\mathrm{~d}, J=16.1,1 \mathrm{H}), 6.22$ (dd, $J=16.0,6.1 \mathrm{~Hz}, 1 \mathrm{H}), 5.91$ (ddd, $J=17.3,10.6,5.7 \mathrm{~Hz}, 1 \mathrm{H}$ ), $5.32(\mathrm{ddd}, J=17.3,1.5,1.5 \mathrm{~Hz}$,

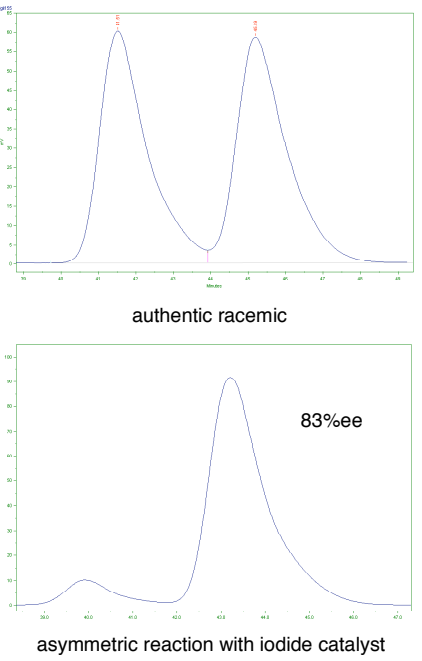

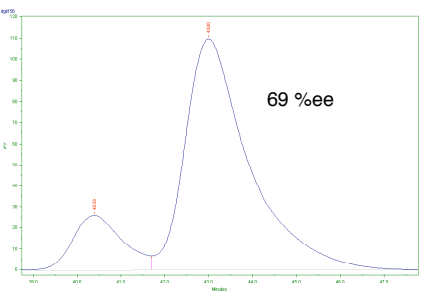

asymmetric reaction with chloride catalyst

Conditions: 98:2 Hex:IPA, Chiracel OD, $1 \mathrm{~mL} / \mathrm{min}, 254 \mathrm{~nm}$ Retention Times: $39.9 \mathrm{~min}, 42.5 \mathrm{~min}$ 
$1 \mathrm{H}), 5.15(\mathrm{ddd}, J=10.6,1.3,1.3 \mathrm{~Hz}, 1 \mathrm{H}), 4.59-4.54(\mathrm{~m}, 1 \mathrm{H}), 4.46-4.42(\mathrm{~m}, 1 \mathrm{H})$, 4.36-4.35 (m, 1H), 1.83-1.61 (m, 4H). $\left.{ }^{13} \mathrm{C} \mathrm{NMR} \mathrm{(100} \mathrm{MHz,} \mathrm{CDCl}_{3}\right)$ : $\square$ 138.99, 137.05, 130.49 , 130.37, 128.61, 127.64, 126.60, 115.41, 72.44, 72.19, 64.57, 38.71, 38.27. HRMS Calcd for $\mathrm{C}_{15} \mathrm{H}_{18} \mathrm{O}_{2}: 230.1307$. Found: 230.1307. Absolute configuration is determined by conversion to $\mathbf{4 a}$.

(2S,4S,6R)-4-Benzyloxytetrahydro-2-styryl-6-vinyl-2H-pyran (13).

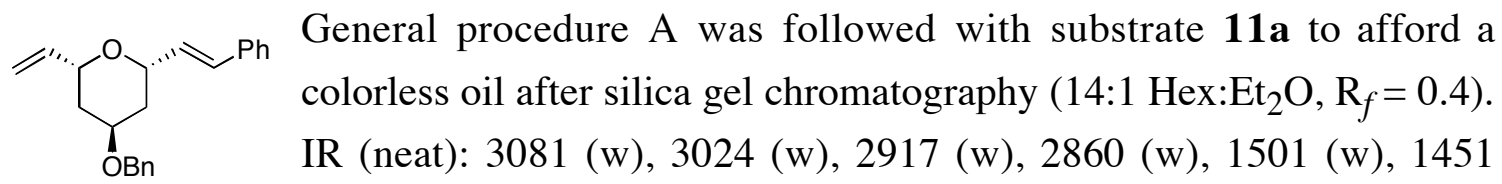
(w), 1338 (m), 1067 (s), 966 (m), 690 (s). ${ }^{1} \mathrm{H}$ NMR (400 MHz, CDCl 3 ): $7.41-7.36$ (m, $6 \mathrm{H}), 7.33-7.28(\mathrm{~m}, 3 \mathrm{H}), 7.24-7.20(\mathrm{~m}, 1 \mathrm{H}), 6.62$ (d, $J=16.0 \mathrm{~Hz}, 1 \mathrm{H}), 6.23$ (dd, $J=16.0$, $6.1 \mathrm{~Hz}, 1 \mathrm{H}), 5.92(\mathrm{ddd}, J=17.3$, 10.6, $5.7 \mathrm{~Hz}, 1 \mathrm{H}), 5.32$ (ddd, $J=$ $17.4,1.5,1.5 \mathrm{~Hz}, 1 \mathrm{H}), 5.15$ (ddd, $J=10.6,1.4,1.4 \mathrm{~Hz}, 1 \mathrm{H}), 4.61$ $(\mathrm{s}, 2 \mathrm{H}), 4.58-4.54(\mathrm{~m}, 1 \mathrm{H})$, 4.56-4.41 (m, 1H), 3.97-3.95 $(\mathrm{m}, 1 \mathrm{H}), 2.04-1.95(\mathrm{~m}, 2 \mathrm{H})$, 1.64-1.51 (m, 2H). ${ }^{13} \mathrm{C}$ NMR

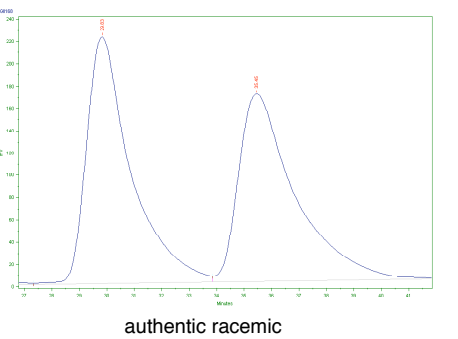
authentic racemic Conditions: 99:1 Hex:IPA, Chiracel AD, $0.2 \mathrm{~mL} / \mathrm{min}, 254 \mathrm{~nm}$ Retention Times: $29.8 \mathrm{~min}, 35.5 \mathrm{~min}$ (100 MHz, $\mathrm{CDCl}_{3}$ ): $\square$ 139.17, $138.95,137.14,130.59,130.37$, 128.60, 128.58, 127.71, 127.59, 127.53, 126.60, 115.28, 72.93,

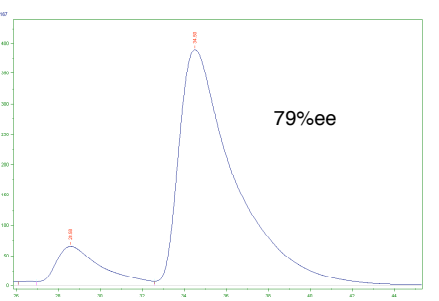

asymmetric reaction with chloride catalyst

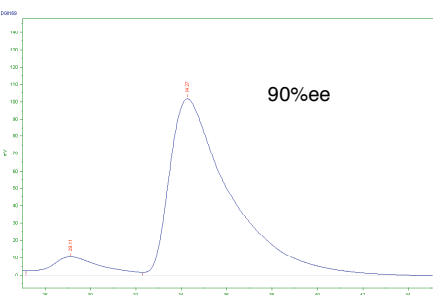

asymmetric reaction with iodide catalyst

72.70, 71.33, 70.40, 35.85, 35.41. HRMS Calcd for $\mathrm{C}_{22} \mathrm{H}_{24} \mathrm{O}_{2} \mathrm{Na}$ : 343.1674. Found: 343.1674. Absolute configuration is determined by conversion to $\mathbf{4 a}$.

\section{4-Benzyloxytetrahydro-2-[(E)-4-(methoxyphenyl)vinyl]-6-vinyl-2H-pyran (14).}

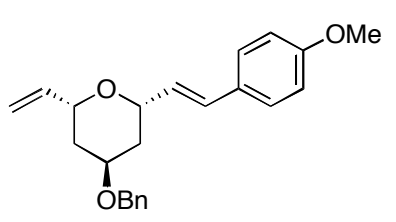

General procedure A was followed with substrate 11b using the chloride catalyst and general procedure B was followed with 11b using the iodide catalyst to afford a colorless oil after silica gel chromatography (19:1 Hex:Et $\left.2 \mathrm{O}, \mathrm{R}_{f}=0.3\right)$. IR (neat): 3024 (w), 2924 (w), 2823 (w), 1608 (m), 1514 (s), 1243 (s), 1174 (m), 1061 (m). ${ }^{1}$ H NMR (400 MHz, $\left.\mathrm{CDCl}_{3}\right)$ : $\square 7.39-7.30(\mathrm{~m}, 5 \mathrm{H}), 7.32(\mathrm{~d}, J=8.8 \mathrm{~Hz}, 2 \mathrm{H}), 6.84(\mathrm{~d}, J=8.8 \mathrm{~Hz}$, $2 \mathrm{H}), 6.56(\mathrm{~d}, J=15.9 \mathrm{~Hz}, 1 \mathrm{H}), 6.10(\mathrm{dd}, J=16.0,6.3 \mathrm{~Hz}, 1 \mathrm{H}), 5.91$ (ddd, $J=17.3,10.6$, 
$5.7 \mathrm{~Hz}, 1 \mathrm{H}$ ), 5.31 (ddd, $J=17.3$, $1.6,1.6 \mathrm{~Hz}, 1 \mathrm{H}), 5.14$ (ddd, $J=$ $10.6,1.5,1.5 \mathrm{~Hz}, 1 \mathrm{H}), 4.60$ (s, $2 \mathrm{H}), \quad 4.55-4.51 \quad(\mathrm{~m}, 1 \mathrm{H})$, 4.45-4.40 (m, 1H), 3.96-3.94 (m, 1H), $3.80(\mathrm{~s}, 3 \mathrm{H}), 2.03-1.94$

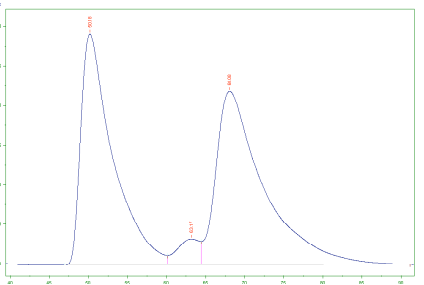

authentic racemic

(m, 2H), $1.63-1.50(\mathrm{~m}, 2 \mathrm{H}) .{ }^{13} \mathrm{C}$ NMR $\left(100 \mathrm{MHz}, \mathrm{CDCl}_{3}\right)$ : 159.26, 139.20, 138.95, 130.02, 129.88, 128.55, 128.34, 127.75, 127.67, 127.50, 115.25, 114.02, 72.91, 72.86, 71.34, 70.35,

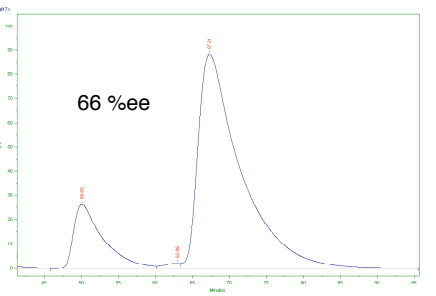

asymmetric reaction using chloride catalyst

Conditions: 99:1 Hex:IPA, Chiracel AD, $0.2 \mathrm{~mL} / \mathrm{min}, 254 \mathrm{~nm}$ Retention Times: $50.1 \mathrm{~min}, 67.3 \mathrm{~min}$

55.37, 35.86, 35.40. HRMS Calcd for $\mathrm{C}_{23} \mathrm{H}_{26} \mathrm{O}_{3} \mathrm{Na}$ : 373.1780. Found: 373.1776 .

\section{4-Benzyloxytetrahydro-2-[(E)-4-(trifluoromethylphenyl)vinyl]-6-vinyl-2H-pyran}

(15).

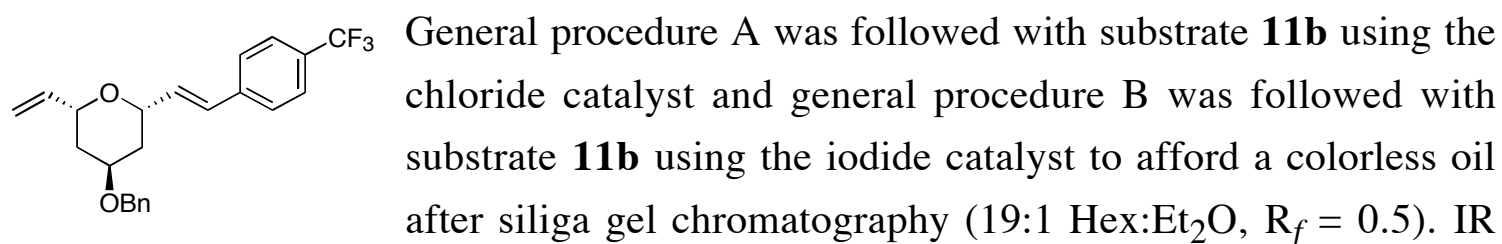
(neat): 3037 (w), 2924 (w), 2861 (w), 1621 (w), 1325 (s), 1168 (m), 1124 (s), 1067 (s). ${ }^{1} \mathrm{H}$ NMR $\left(400 \mathrm{MHz}, \mathrm{CDCl}_{3}\right)$ : $7.55(\mathrm{~d}, J=8.2 \mathrm{~Hz}, 2 \mathrm{H}), 7.46(\mathrm{~d}, J=8.3 \mathrm{~Hz}, 2 \mathrm{H})$, 7.39-7.36 (m, 4H), 7.33-7.30 (m, 1H), $6.66(\mathrm{~d}, J=16.0 \mathrm{~Hz}, 1 \mathrm{H}), 6.32(\mathrm{dd}, J=16.0,5.7$ $\mathrm{Hz}, 1 \mathrm{H}), 5.92$ (ddd, $J=17.3,10.6,5.7 \mathrm{~Hz}, 1 \mathrm{H}), 5.32$ (ddd, $J=17.3,1.5,1.5 \mathrm{~Hz}, 1 \mathrm{H}$ ), 5.16 (ddd, $J=10.6,1.3,1.3 \mathrm{~Hz}, 1 \mathrm{H}), 4.61(\mathrm{~s}, 2 \mathrm{H}), 4.60-4.56(\mathrm{~m}, 1 \mathrm{H}), 4.45-4.41(\mathrm{~m}, 1 \mathrm{H})$, $3.98-3.96(\mathrm{~m}, 1 \mathrm{H}), 2.04-1.96$ $(\mathrm{m}, 2 \mathrm{H}), 1.62-1.50(\mathrm{~m}, 2 \mathrm{H}) .{ }^{13} \mathrm{C}$ NMR $\left(100 \mathrm{MHz}, \mathrm{CDCl}_{3}\right)$ : 140.87, 139.21, 139.07, 133.53, $129.73,129.40,128.97,128.80$, 127.96, 127.74, 126.90, 125.83 (q, $J=3.8 \mathrm{~Hz}), 115.60,73.19$, $72.53,71.41,70.65,36.00$, 35.55. HRMS Calcd for $\mathrm{C}_{23} \mathrm{H}_{23} \mathrm{O}_{2} \mathrm{~F}_{3} \mathrm{Na}$ : $\quad 411.1548$. Found: 411.1553 .

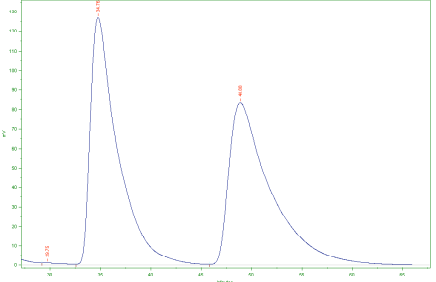

authentic racemic

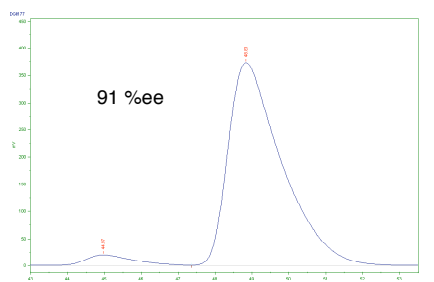

asymmetric reaction with iodide catalyst

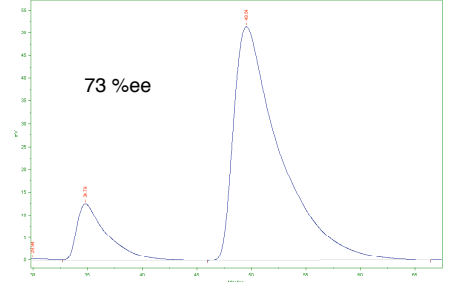

asymmetric reaction with chloride catalyst

Conditions: 99:1 Hex:IPA, Chiracel AD, $0.2 \mathrm{~mL} / \mathrm{min}, 254 \mathrm{~nm}$ Retention Times: $34.5 \mathrm{~min}, 48.5 \mathrm{~min}$ 


\section{4-Benzyloxy-2-[(E)-2-Cyclohexylvinyl]tetrahydro-6-vinyl-2H-pyran (16).}

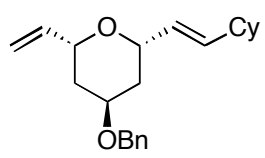

General procedure B was used with $\mathbf{1 1 b}$ to afford $\mathbf{1 6}$ as a colorless oil after silica gel chromatography (19:1 Hex:Et $\left.{ }_{2} \mathrm{O} \mathrm{R}_{f}=0.5\right)$. IR (neat): 2917 (s), 2848 (m), 1451 (m), 1338 (w), 1061 (s), 966 (m), 702 (w). ${ }^{1} \mathrm{H}$ NMR (400 MHz, $\mathrm{CDCl}_{3}$ ): $\square 7.38-7.35$ (m, 4H), 7.31-7.27 (m, 1H), 5.88 (ddd, $J=17.3$, $10.6,5.8 \mathrm{~Hz}, 1 \mathrm{H}), 5.64$ (dd, $J=15.7,6.5 \mathrm{~Hz}, 1 \mathrm{H}), 5.43$ (dd, $J=15.7,6.4 \mathrm{~Hz}, 1 \mathrm{H}), 5.25$ $(\mathrm{d}, J=17.3,1 \mathrm{H}), 5.10(\mathrm{~d}, J=10.6 \mathrm{~Hz}, 1 \mathrm{H}), 4.57$ (s, 2H), 4.36-4.28 (m, 2H), 3.91-3.89 $(\mathrm{m}, 1 \mathrm{H}), 1.93-1.86(\mathrm{~m}, 3 \mathrm{H})$, $1.72-1.69(\mathrm{~m}, 5 \mathrm{H}), 1.52-1.45$ $(\mathrm{m}, 2 \mathrm{H}), 1.27-1.04(\mathrm{~m}, 5 \mathrm{H}) .{ }^{13} \mathrm{C}$ NMR (100 $\left.\mathrm{MHz}, \mathrm{CDCl}_{3}\right): \square$ 139.39, 139.04, 137.92, 128.54, $128.42,127.64,127.50,115.14$, 72.92, 72.86, 71.41, 70.28, 40.48, 35.91, 35.39, 32.85, 26.35, 26.21. HRMS Calcd for $\mathrm{C}_{22} \mathrm{H}_{30} \mathrm{O}_{2}$ : 326.2246. Found: 326.2250. The ee was determined by HPLC analysis
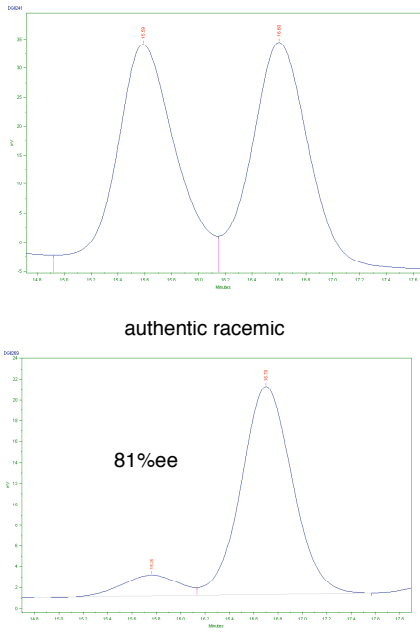

asymmetric reactıon usıng ıoaıde catalyst

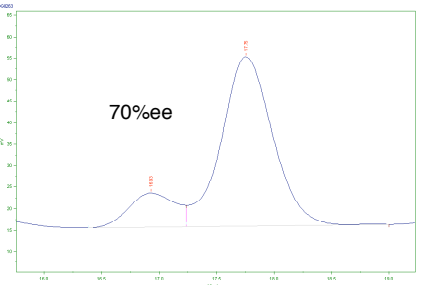

asymmetric reaction using chloride catalyst

Conditions: 98:2 Hex:IPA, Chiralpak AS, $0.5 \mathrm{~mL} / \mathrm{min}, 210 \mathrm{~nm}$ Retention Times: $15.8 \mathrm{~min}, 16.7 \mathrm{~min}$

of the debenzylated alcohol of 16. Debenzylation was carried out under dissolving metal conditions using calcium.

\section{$(2 S, 4 S, 6 R)-4-[($ tert-Butyldimethylsilyl)oxy] tetrahydro-2-styryl-6-vinyl-2H-pyran}

(17).

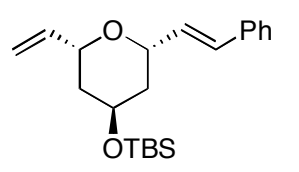

General procedure A was followed with 11d using chloride catalyst and general procedure B was followed with 11d using the iodide catalyst to afford a colourless oil after silica gel chromatography (24:1

Hex:Et ${ }_{2} \mathrm{O}, \mathrm{R}_{f}=0.3$ ). IR (neat): 3024 (w), 2949 (s), 2923 (s), 2855 (m), 1243 (m), 1092 (s), 1055 (s), 909 (m), 835 (s), 771 (m), 690 (m). ${ }^{1} \mathrm{H}$ NMR (400 MHz, $\mathrm{CDCl}_{3}$ ): 7.39-7.37 (m, 2H), 7.31-7.28 (m, 2H), 7.23-7.20 (m, 1H), 6.61 (d, $J$ $=16.0 \mathrm{~Hz}, 1 \mathrm{H}), 6.24(\mathrm{dd}, J=$ $16.0,6.0 \mathrm{~Hz}, 1 \mathrm{H}), 5.91$ (ddd, $J=$ 16.7, 10.6, $5.6 \mathrm{~Hz}, 1 \mathrm{H}), 5.30$ (dd, $J=16.7,1.0 \mathrm{~Hz}, 1 \mathrm{H}), 5.13(\mathrm{dd}, J$

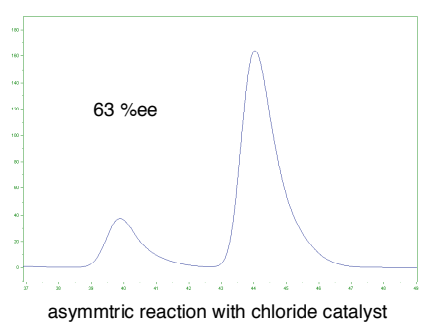

asymmtric reaction with chloride catalyst

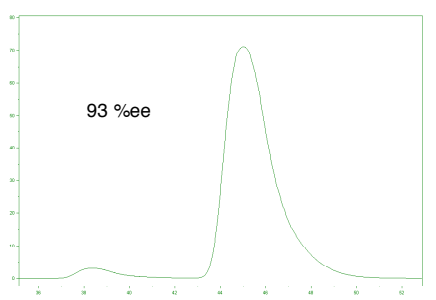

asymmetric reaction with iodide catalyst 
$=10.6,0.9 \mathrm{~Hz}, 1 \mathrm{H}), 4.59-4.54(\mathrm{~m}, 1 \mathrm{H}), 4.46-4.42(\mathrm{~m}, 1 \mathrm{H}), 4.28-4.27(\mathrm{~m}, 1 \mathrm{H})$, 1.73-1.55 (m, 4H), 0.94 (s, 9H), 0.09 (s, 6H). ${ }^{13} \mathrm{C}$ NMR (100 MHz, $\mathrm{CDCl}_{3}$ ): $\square$ 139.44, 137.22, 130.90, 130.04, 128.60, 127.53, 126.59, 114.97, 72.57, 72.30, 64.96, 39.50, 39.08, 25.97, 18.23, 14.47. HRMS Calcd for $\mathrm{C}_{21} \mathrm{H}_{32} \mathrm{O}_{2} \mathrm{Si}$ : 344.2172. Found: 344.2178.

The enantiomeric excess was determined by deprotection in $10 \% \mathrm{HCl}_{(\mathrm{aq})} / \mathrm{THF}$ followed by HPLC using the conditions reported for alcohol 11a. Absolute configuration is determined by conversion to $\mathbf{4 a}$.

\section{Tetrahydro-4-methyl-2-(E)-styryl-6-vinyl-2H-pyran-4-ol (19).}

Me $\mathrm{OH} \quad$ General procedure A was followed with substrate $\mathbf{1 8}$ to afford a colourless oil after silica gel chromatography $\left(3: 1 \mathrm{Hex}: \mathrm{Et}_{2} \mathrm{O}, \mathrm{R}_{f}=\right.$ Ph 0.15). IR (neat): 3398 (br), 2971 (m), 2938 (m), 2918 (m), 1376 (m), $1103(\mathrm{~m}), 1070(\mathrm{~m}), 966(\mathrm{~m}), 746(\mathrm{~m}), 694(\mathrm{~m}) .{ }^{1} \mathrm{H} \mathrm{NMR}\left(400 \mathrm{MHz}, \mathrm{CDCl}_{3}\right)$ : 7.41-7.20 (m, 5H), 6.63 (d, $J=15.9 \mathrm{~Hz}, 1 \mathrm{H}), 6.22(\mathrm{~d}, J=15.9,6.0 \mathrm{~Hz}, 1 \mathrm{H}), 5.91$ (ddd, $J$ $=17.2,10.6,5.7 \mathrm{~Hz}, 1 \mathrm{H}), 5.32$ (ddd, $J=17.2,1.3,1.3 \mathrm{~Hz}, 1 \mathrm{H}$ ), 5.18 (ddd, $J=10.6,1.3,1.3 \mathrm{~Hz}$, $1 \mathrm{H}), \quad 4.17-4.06 \quad(\mathrm{~m}, \quad 1 \mathrm{H})$, 4.03-3.94 (m, 1H), 1.82 (ddd, $J$ $=12.1,2.4,2.4 \mathrm{~Hz}, 1 \mathrm{H}), 1.77$ (ddd, $J=12.1,2.4,2.4 \mathrm{~Hz}, 1 \mathrm{H}$ ), $1.58(\mathrm{dd}, J=12.1,12.1 \mathrm{~Hz}, 1 \mathrm{H})$, $1.52(\mathrm{dd}, J=12.1,12.1 \mathrm{~Hz}, 1 \mathrm{H})$, $1.51(\mathrm{br} \mathrm{s}, 1 \mathrm{H}), 1.42(\mathrm{~s}, 3 \mathrm{H}$, $\left.\mathrm{CH}_{3}\right) .{ }^{13} \mathrm{C}$ NMR $(100 \mathrm{MHz}$, $\left.\mathrm{CDCl}_{3}\right):$ 138.46, 136.88, 130.80, 129.75, 128.65, 127.78,

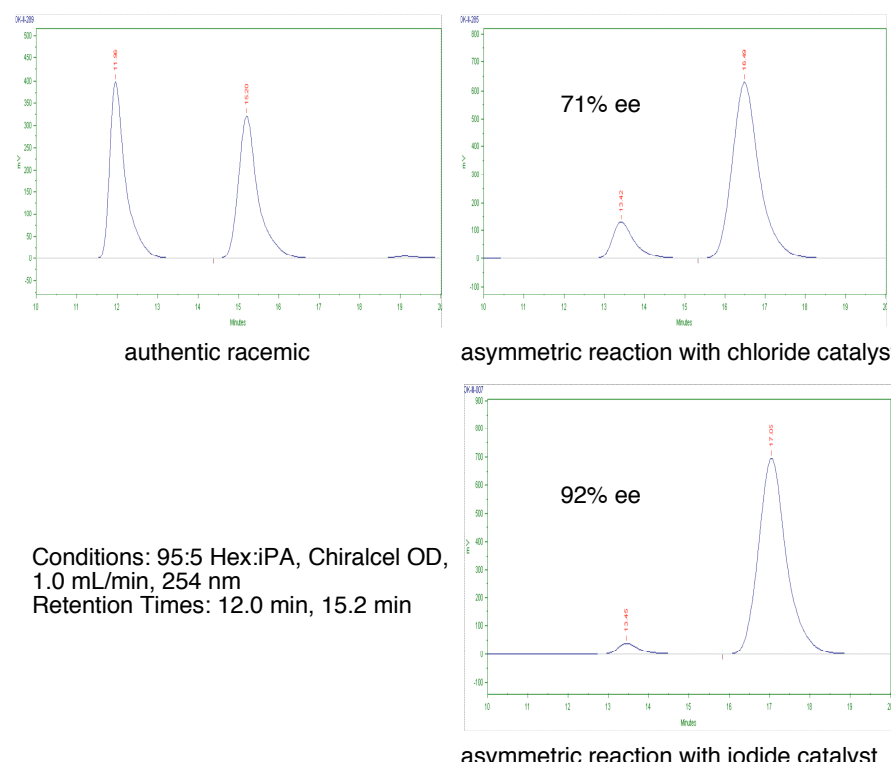

asymmetric reaction with iodide catalyst 126.65, 115.71, 75.91, 75.67, 69.40, 46.24, 45.83, 26.01. HRMS Calcd for $\mathrm{C}_{16} \mathrm{H}_{20} \mathrm{O}_{2}$ : 244.1463. Found: 244.1460 . 
Tetrahydro-2-[(E)-2-(4-methoxyphenyl)vinyl]-4-methyl-6-vinyl-2H-pyran-4-ol (20).

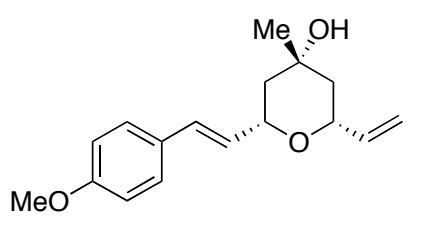

General procedure A was followed with $\mathbf{1 8}$ to afford a colourless oil after silica gel chromatography (Hex: $\mathrm{Et}_{2} \mathrm{O}=3: 1$, $\left.\mathrm{R}_{f}=0.15\right) . \quad \mathrm{IR}$ (neat): 3413 (br), 2936 (m), 2837 (m), 1607 (m), $1511(\mathrm{~m}), 1250(\mathrm{~m}), 1175(\mathrm{~m}), 1106(\mathrm{~m}), 1034(\mathrm{~m}), 968$ (m), $923(\mathrm{~m}), 814(\mathrm{~m}) .{ }^{1} \mathrm{H}$ NMR (400 MHz, $\left.\mathrm{CDCl}_{3}\right)$ : $\square 7.31(\mathrm{~d}, J=8.6 \mathrm{~Hz}, 2 \mathrm{H}), 6.84$ (d, $J=8.6 \mathrm{~Hz}, 2 \mathrm{H}), 6.57(\mathrm{~d}, J=15.9 \mathrm{~Hz}, 1 \mathrm{H}), 6.08$ (dd, $J=15.9,6.2 \mathrm{~Hz}, 1 \mathrm{H}), 5.91$ (ddd $J=$ $17.2,10.6,5.7 \mathrm{~Hz}, 1 \mathrm{H}), 5.31(\mathrm{~d}$, $J=17.2 \mathrm{~Hz}, 1 \mathrm{H}), 5.16(\mathrm{~d}, J=$ $10.6 \mathrm{~Hz}, 1 \mathrm{H}), 4.08$ (dd, $J=11.4$, $6.2 \mathrm{~Hz}, 1 \mathrm{H}), 3.97(\mathrm{dd}, J=11.4$, $5.7 \mathrm{~Hz}, 1 \mathrm{H}), 3.80(\mathrm{~s}, 3 \mathrm{H})$, 1.84-1.72 (m, 2H), 1.63-1.43 (m, 2H), 1.51 (br s, 1H), 1.42 (s,

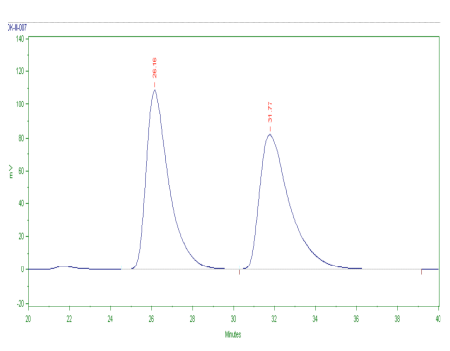

authentic racemic

$3 \mathrm{H}) .{ }^{13} \mathrm{C}$ NMR $(100 \mathrm{MHz}$, $\left.\mathrm{CDCl}_{3}\right)$ : $\square \quad$ 159.41, 138.52, 130.48, 129.63, 127.85, 127.55, 115.71, 114.08, 75.91, 75.88, $69.42 ， 55.41 ， 46.33 ， 45.82$, 26.02. HRMS Calcd for $\mathrm{C}_{17} \mathrm{H}_{22} \mathrm{O}_{3}$ : 274.1569. Found:

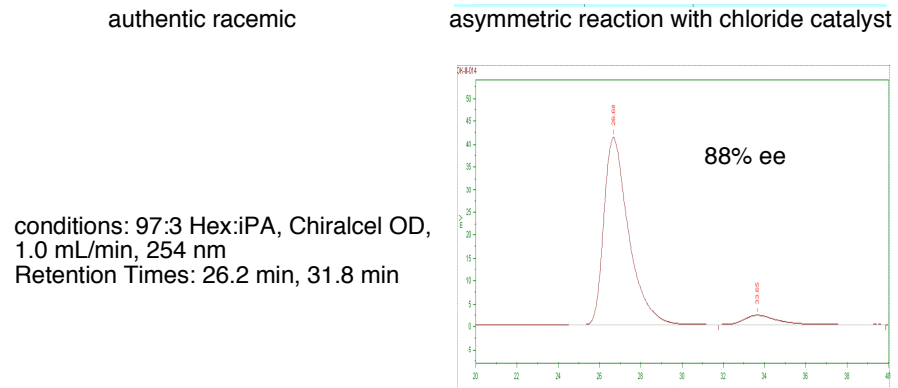

asymmetric reaction with iodide catalyst

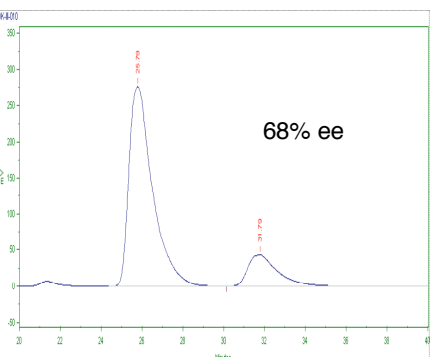

asymmetric reaction with chloride catalyst 274.1563 .

\section{2-[(E)-2-(4-trifluoromethylphenyl)vinyl]tetrahydro-4-methyl-6-vinyl-2H-pyran-4-ol} (21).

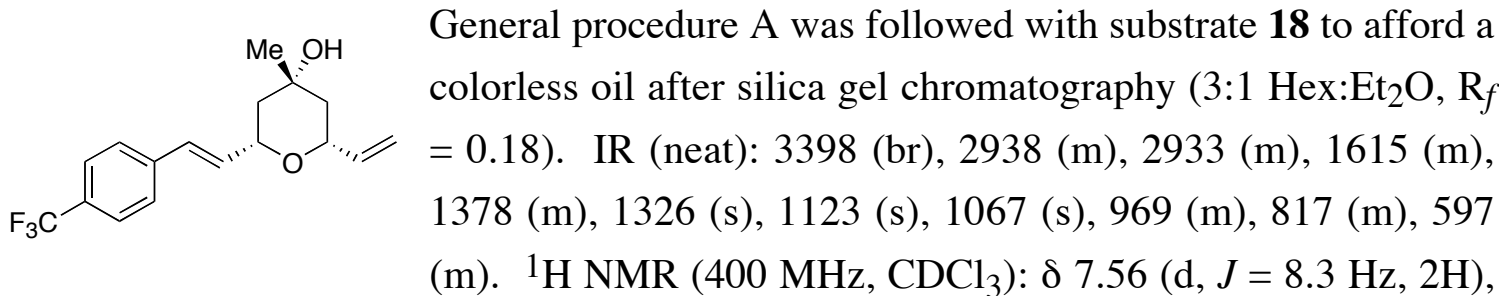

$7.46(\mathrm{~d}, J=8.3 \mathrm{~Hz}, 2 \mathrm{H}), 6.67(\mathrm{~d}, J=16.1 \mathrm{~Hz}, 1 \mathrm{H}), 6.31(\mathrm{dd}, J=16.1,5.5 \mathrm{~Hz}, 1 \mathrm{H}), 5.91$ (ddd, $J=17.2,10.6,5.7 \mathrm{~Hz}, 1 \mathrm{H}$ ), 5.33 (ddd, $J=17.2,1.5,1.5 \mathrm{~Hz}, 1 \mathrm{H}$ ), 5.18 (ddd, $J=$ $10.6,1.5,1.5 \mathrm{~Hz}, 1 \mathrm{H}$ ), 4.14 (dddd, $J=11.4,5.5,1.7,1.7 \mathrm{~Hz}, 1 \mathrm{H}$ ), 3.99 (ddddd, $J=11.7$, 5.7, 2.0, 1.1, $1.1 \mathrm{~Hz}, 1 \mathrm{H}), 1.83$ (ddd, $J=12.6,2.4,2.4 \mathrm{~Hz}, 1 \mathrm{H}), 1.78$ (ddd, $J=12.6,2.4$, $2.4 \mathrm{~Hz}, 1 \mathrm{H}), 1.62-1.48(\mathrm{~m}, 2 \mathrm{H}), 1.57$ (br s, $1 \mathrm{H}), 1.43$ (s, 3H). ${ }^{13} \mathrm{C} \mathrm{NMR}(100 \mathrm{MHz}$, $\left.\mathrm{CDCl}_{3}\right):[140.43,138.30,132.43,129.20,126.77,125.64(\mathrm{q}, J=3.8 \mathrm{~Hz}), 115.85,76.00$, 
$75.25,69.35,46.05,45.81$, 25.99. ${ }^{19} \mathrm{~F}$ NMR $(376 \mathrm{MHz}$, $\left.\mathrm{CDCl}_{3}\right): 0-63.01$. HRMS Calcd for $\mathrm{C}_{17} \mathrm{H}_{19} \mathrm{~F}_{3} \mathrm{O}_{2}$ : 312.1337. Found: 312.1341.

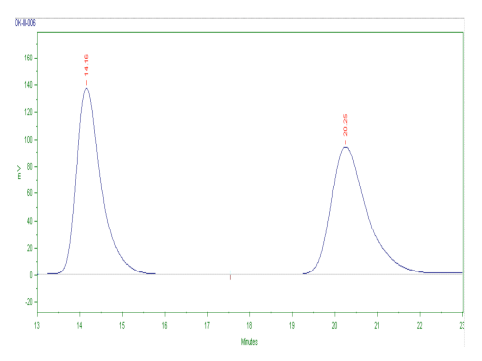

authentic racemic

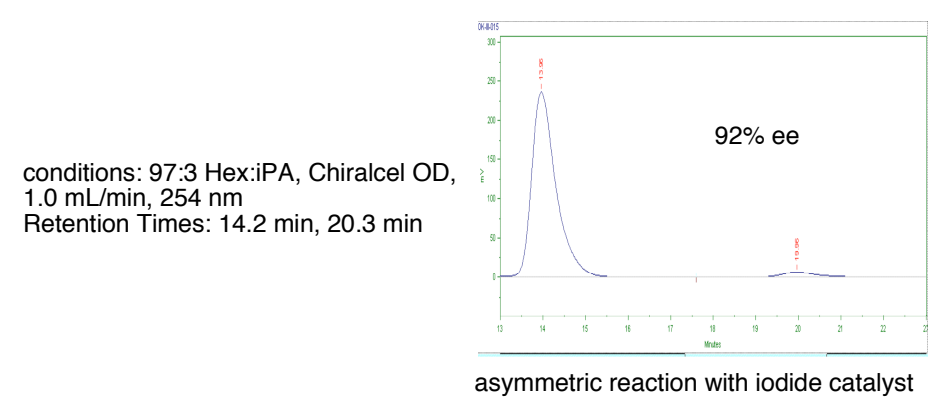

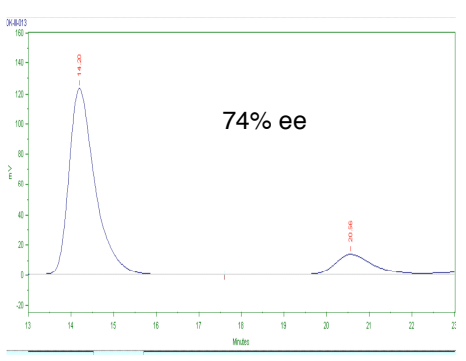

asymmetric reaction with chloride catalyst

\section{Tetrahydro-2-(E)-styryl-6-vinyl-2H-pyran (23).}

General procedure A was followed with substrate 22 to afford a $\mathrm{Ph} \widehat{N}_{\mathrm{O}} \ldots$ colourless oil after silica gel chromatography $\left(3: 1 \mathrm{Hex}: \mathrm{CH}_{2} \mathrm{Cl}_{2}, \mathrm{R}_{f}=\right.$ 0.10). IR (neat): 2935 (m), 2855 (w), 1495 (w), 1304 (w), 1198 (w), $1073(\mathrm{~m}), 965$ (m), $746(\mathrm{~m}), 693(\mathrm{~m}) .{ }^{1} \mathrm{H}$ NMR (400 MHz, $\left.\mathrm{CDCl}_{3}\right)$ : $7.40-7.35$ (m, $2 \mathrm{H}), 7.31-7.18(\mathrm{~m}, 3 \mathrm{H}), 6.61(\mathrm{dd}, J=15.9 .1 .1 \mathrm{~Hz}, 1 \mathrm{H}), 6.25(\mathrm{dd}, J=15.9,6.0 \mathrm{~Hz}, 1 \mathrm{H})$, 5.93 (ddd, $J=17.4,10.6,5.5 \mathrm{~Hz}, 1 \mathrm{H}), 5.29$ (ddd, $J=17.4,1.5,1.5 \mathrm{~Hz}, 1 \mathrm{H}$ ), 5.12 (ddd, $J$ $=10.6,1.5,1.5 \mathrm{~Hz}, 1 \mathrm{H})$,

4.11-4.04 (m, $1 \mathrm{H})$, 3.98-3.91 (m, 1H), 1.98-1.89 (m, 1H),

1.77-1.58 (m, $3 \mathrm{H})$, $1.55-1.30 \quad(\mathrm{~m}, \quad 2 \mathrm{H}) .{ }^{13} \mathrm{C}$ NMR (100 MHz, $\left.\mathrm{CDCl}_{3}\right)$ : $139.48,137.22,130.95$, $130.03,128.61,127.55$, 126.61, 114.96, 78.53, 78.24, 31.77, 31.32, 23.61. conditions: 99.9:0.1 Hex:iPA, Chiralcel OJ, Retention Times: $98.0 \mathrm{~min}, 112.0 \mathrm{~min}$

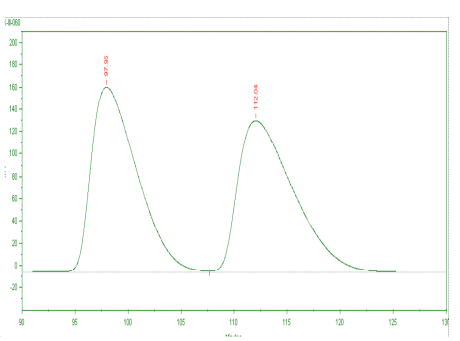

authentic racemic

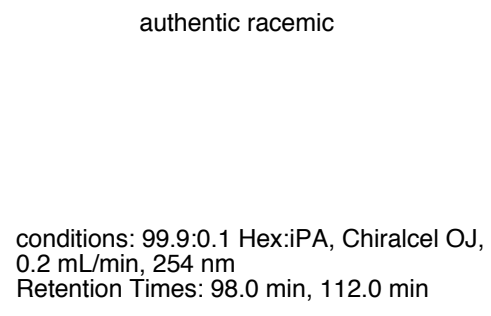

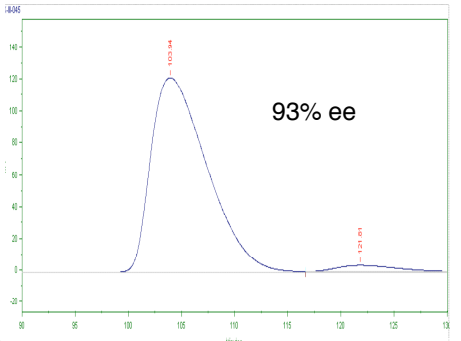

asymmetric reaction with chloride catalyst

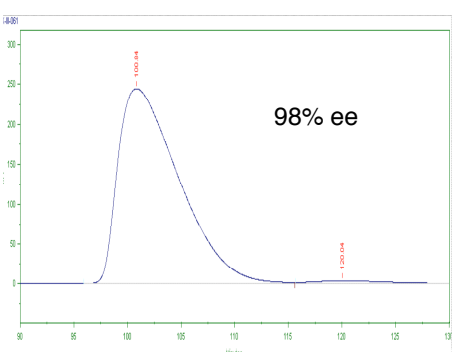

asymmetric reaction with iodide catalyst 
Tetrahydro-4-methylidene-2-(E)-styryl-6-vinyl-2H-pyran (25).

General procedure A was followed with $\mathbf{2 4}$ to afford a colorless oil after silica gel chromatography (3:1 Hex: $\left.\mathrm{CH}_{2} \mathrm{Cl}_{2}, \mathrm{R}_{f}=0.15\right)$. IR (neat): $3025(\mathrm{~m}), 2941(\mathrm{~m}), 1653(\mathrm{~m}), 1449(\mathrm{~m}), 1309(\mathrm{~m}), 1061(\mathrm{~s})$, 893 (m), 747 (s), 693 (s). ${ }^{1} \mathrm{H}$ NMR (400 MHz, $\left.\mathrm{CDCl}_{3}\right)$ : $\square 7.42-7.19$ (m, 5H), 6.63 (d, $J=$ $15.9 \mathrm{~Hz}, 1 \mathrm{H}), 6.27$ (d, $J=15.9,6.0 \mathrm{~Hz}, 1 \mathrm{H}), 5.95$ (ddd, $J=17.4,10.4,6.0 \mathrm{~Hz}, 1 \mathrm{H}$ ), $5.37-5.29(\mathrm{~m}, 1 \mathrm{H}), 5.21-5.14$ $(\mathrm{m}, 1 \mathrm{H}), 4.82(\mathrm{~d}, J=1.7 \mathrm{~Hz}$, $1 \mathrm{H}), 4.82(\mathrm{~d}, J=1.7 \mathrm{~Hz}, 1 \mathrm{H})$, 4.08-4.00 (m, 1H), 3.95-3.85 $(\mathrm{m}, 1 \mathrm{H}), 2.41-2.28(\mathrm{~m}, 2 \mathrm{H})$, 2.24-2.07 (m, 2H). ${ }^{13} \mathrm{C}$ NMR $\left(100 \mathrm{MHz}, \mathrm{CDCl}_{3}\right):$ 143.82, 138.67, 136.96, 130.78, 130.00, $128.65,127.75,126.67,115.71$, 109.48, 79.11, 78.90, 40.96,

conditions: 99.8:0.2 Hex:iPA, Chiralcel OB-H, $0.5 \mathrm{~mL} / \mathrm{min}, 254 \mathrm{~nm}$ Retention Times: $36.1 \mathrm{~min}, 56.1 \mathrm{~min}$

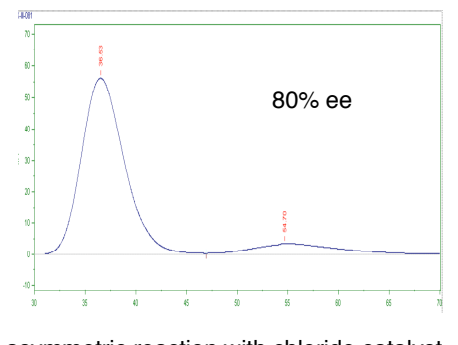

asymmetric reaction with chloride catalyst

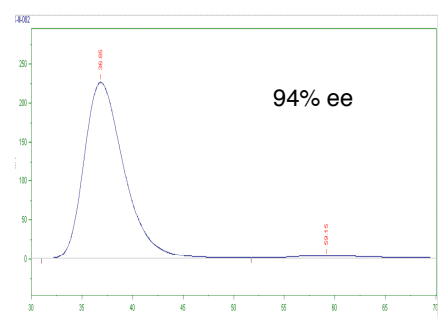

asymmetric reaction with iodide catalyst

$\mathrm{C}_{16} \mathrm{H}_{18} \mathrm{O}$ : 226.1358. Found: 226.1352 .

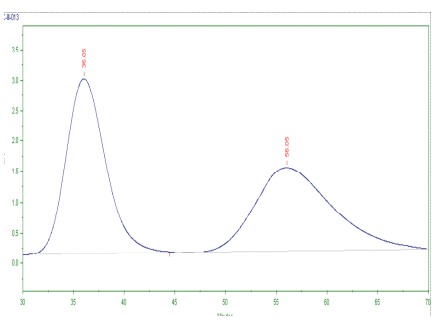

authentic racemic

\section{Tetrahydro-4-iodo-2-(E)-styryl-6-vinyl-2H-pyran (27).}

colorless solid

y (3:1 Hex: $\left.\mathrm{CH}_{2} \mathrm{Cl}_{2}, \mathrm{R}_{f}=0.10\right)$.

IR (neat): 3024 (m), 2949 (m), $2888(\mathrm{~m}), 1495(\mathrm{~m}), 1412(\mathrm{~m})$, 1308 (m), 1236 (m), 1057 (s), 965 (m), 746 (s), 692 (s). ${ }^{1} \mathrm{H}$ NMR $\left(400 \mathrm{MHz}, \mathrm{CDCl}_{3}\right): \square 7.42-7.20$ $(\mathrm{m}, 5 \mathrm{H}), 6.66(\mathrm{~d}, J=16.1 \mathrm{~Hz}$, $1 \mathrm{H}), 6.25(\mathrm{dd}, J=16.1,5.9 \mathrm{~Hz}$, 1H), 5.94 (ddd, $J=17.4,10.6,5.5$ $\mathrm{Hz}, 1 \mathrm{H}$ ), 5.35 (ddd, $J=17.4,1.5$, $1.5 \mathrm{~Hz}, 1 \mathrm{H}), 5.19$ (ddd, $J=10.6$, $1.5,1.5 \mathrm{~Hz}, 1 \mathrm{H}), 4.92$ (dddd, $J=$

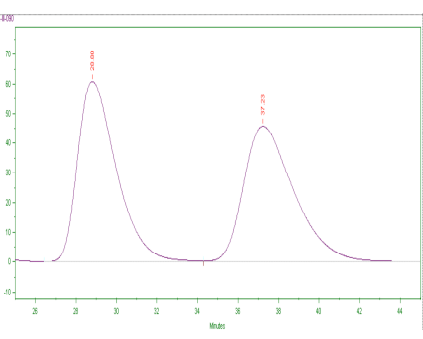

authentic racemic

conditions: 99.8:0.2 Hex:iPA, Chiralcel OJ, $1.0 \mathrm{~mL} / \mathrm{min}, 254 \mathrm{~nm}$ Retention Times: $28.8 \mathrm{~min}, 37.2 \mathrm{~min}$ asymmetric reaction with chloride catalyst

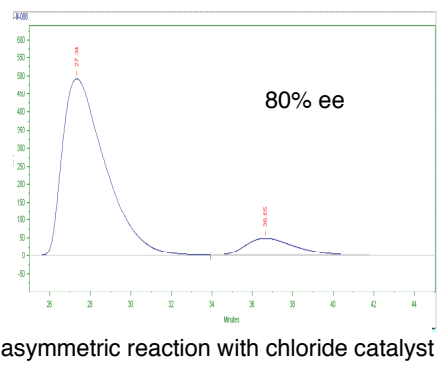

$93 \%$ ee

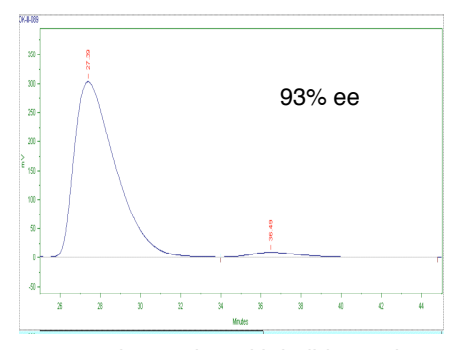


$3.1,3.1,3.1,3.1 \mathrm{~Hz}, 1 \mathrm{H}), 4.64-4.55(\mathrm{~m}, 1 \mathrm{H}), 4.51-4.43(\mathrm{~m}, 1 \mathrm{H}), 2.13$ (dddd, $J=14.7$, 2.2, 2.2, $2.2 \mathrm{~Hz}, 1 \mathrm{H}), 2.09$ (dddd, $J=14.7,2.2,2.2,2.2 \mathrm{~Hz}, 1 \mathrm{H}), 1.71$ (ddd, $J=14.7$, 10.6, $3.7 \mathrm{~Hz}, 1 \mathrm{H}), 1.65$ (ddd, $J=14.7,10.6,3.7 \mathrm{~Hz}, 1 \mathrm{H}) .{ }^{13} \mathrm{C} \mathrm{NMR}\left(100 \mathrm{MHz}, \mathrm{CDCl}_{3}\right)$ : 137.84, 136.84, 131.10, 129.12, 128.67, 127.82, 126.65, 116.06, 74.31, 74.16, 40.68, 40.24, 29.59. HRMS Calcd for $\mathrm{C}_{15} \mathrm{H}_{17} \mathrm{IO}: 340.0324$. Found: 340.0326 .

\section{(2S,4R,6R)-Tetrahydro-4-(triisopropylsilyloxy)-2-(E)-styryl-6-vinyl-2H-pyran.}

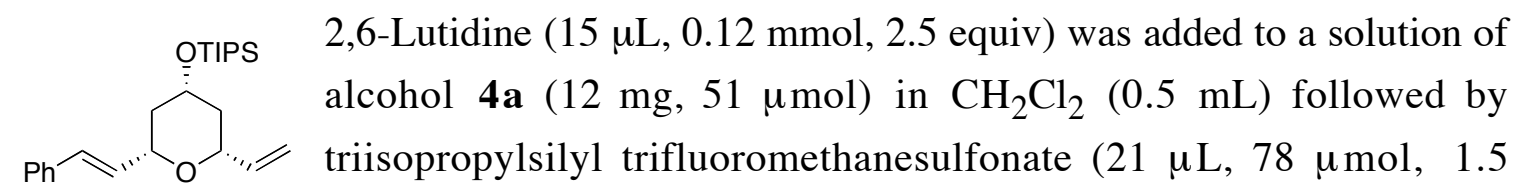
equiv) at $-78{ }^{\circ} \mathrm{C}$. After stirring for $2 \mathrm{~h}$, the reaction mixture was treated with saturated aqueous $\mathrm{NaHCO}_{3}$, warmed to $22{ }^{\circ} \mathrm{C}$, and the organic phase was separated. The aqueous layer was extracted with EtOAc, and the combined organic layers were washed with brine, dried $\left(\mathrm{MgSO}_{4}\right)$, filtered, and concentrated to dryness. Silica gel chromatography of the residue (50:1 Hex: $\left.\mathrm{Et}_{2} \mathrm{O}\right)$ afforded silyl ether $(17 \mathrm{mg}, 45 \square \mathrm{mol}, 88 \%)$ as a colourless oil. IR (neat): 2943 (m), 2866 (m), 1463 (m), 1381 (m), 1125 (m), 1068 (m), 964 (m), $883(\mathrm{~m}), 691(\mathrm{~m}) .{ }^{1} \mathrm{H}$ NMR (400 MHz, $\left.\mathrm{CDCl}_{3}\right)$ : $\square 7.41-7.19(\mathrm{~m}, 5 \mathrm{H}), 6.61$ (d, $J=16.1$ $\mathrm{Hz}, 1 \mathrm{H}), 6.25$ (dd, $J=16.1,6.0 \mathrm{~Hz}, 1 \mathrm{H}), 5.93$ (ddd, $J=17.2,10.4,5.9 \mathrm{~Hz}, 1 \mathrm{H}), 5.31$ (ddd, $J=17.2,1.3,1.3 \mathrm{~Hz}, 1 \mathrm{H}), 5.15$ (ddd, $J=10.4,1.3,1.3 \mathrm{~Hz}, 1 \mathrm{H}), 4.10-3.88$ (m, 3H), 2.03 (dddd, $J=12.6,4.6,2.0,2.0 \mathrm{~Hz}, 1 \mathrm{H}$ ), 1.99 (dddd, $J=12.6,4.6,2.0,2.0 \mathrm{~Hz}, 1 \mathrm{H}$ ), $1.47(\mathrm{ddd}, J=11.7,11.7,11.7 \mathrm{~Hz}, 1 \mathrm{H}), 1.41(\mathrm{ddd}, J=11.7,11.7,11.7 \mathrm{~Hz}, 1 \mathrm{H})$, $1.18-1.10(\mathrm{~m}, 21 \mathrm{H}) .{ }^{13} \mathrm{C} \mathrm{NMR}\left(100 \mathrm{MHz}, \mathrm{CDCl}_{3}\right)$ : $\square 138.57,136.99,130.60,129.91$, 128.63, 127.71, 126.65, 115.52, 76.56, 76.30, 68.74, 42.01, 41.59, 18.25, 12.51. HRMS Calcd for $\mathrm{C}_{24} \mathrm{H}_{38} \mathrm{O}_{2} \mathrm{Si}: 386.2641$. Found: 386.2639 .

\section{$(2 R, 4 R, 6 S)$-Tetrahydro-2-(2-hydroxyethyl)-4-(triisopropylsilyloxy)-6-(E)-styryl-2H-} pyran.

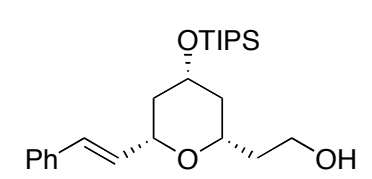

A solution of diene $(37.5 \mathrm{mg}, 97.0 \square \mathrm{mol})$ in THF $(1.0 \mathrm{~mL})$ was added to a solution of $9-\mathrm{BBN}$ (14.5 $\mathrm{mg}, 0.119 \mathrm{mmol}, 1.2$ equiv) in mixture was cooled to $0{ }^{\circ} \mathrm{C}$. EtOH/THF $(1: 1,0.2 \mathrm{~mL}$ ), pH 7.00 buffer (Fisher; $0.05 \mathrm{M}$ potassium phosphate monobasic/sodium hydroxide, $0.2 \mathrm{~mL})$, and then $30 \% \mathrm{H}_{2} \mathrm{O}_{2}(0.2$ $\mathrm{mL}$ ) were added successively, and the mixture was stirred at $22^{\circ} \mathrm{C}$ for $12 \mathrm{~h}$. The reaction mixture was diluted with brine $(1 \mathrm{~mL})$ and extracted with EtOAc $(5 \mathrm{~mL} \times 3)$. The combined organic layers were dried, filtered, and concentrated. The residue was purified by silica gel chromatography (9:1 Hex:EtOAc, $\left.\mathrm{R}_{f}=0.18\right)$ to give the alcohol $(33.3 \mathrm{mg}$, 
82.3 ঢmol, 85\%) as a colorless oil. IR (neat): 3414 (br), 2943 (m), 2866 (m), 1462 (m), $1384(\mathrm{~m}), 1248(\mathrm{~m}), 1085(\mathrm{~m}), 883(\mathrm{~m}), 690(\mathrm{~m}) .{ }^{1} \mathrm{H}$ NMR (400 MHz, CDCl 3 ): $7.40-7.20(\mathrm{~m}, 5 \mathrm{H}), 6.56(\mathrm{~d}, J=15.9 \mathrm{~Hz}, 1 \mathrm{H}), 6.20(\mathrm{dd}, J=15.9,6.0 \mathrm{~Hz}, 1 \mathrm{H}), 4.06-3.90$ (m, 2H), 3.90-3.72 (m, 3H), 3.72-3.62 (m, 1H), 2.06-1.98 (m, 1H), 1.94-1.36 (m, 5H), $1.16-0.98(\mathrm{~m}, 21 \mathrm{H}) .{ }^{13} \mathrm{C} \mathrm{NMR}\left(100 \mathrm{MHz}, \mathrm{CDCl}_{3}\right): \square 136.66,130.82,129.42,128.65$, $127.86,126.65,76.78,72.83,68.39,61.68,41.95,41.75,37.64,34.60,18.21,12.45$.

(2S,4R,6R)-Tetrahydro-4-(triisopropylsilyloxy)-2-[2-(4-methoxybenzyloxy)ethyl]-6(E)-styryl-2H-pyran (27).

A solution of the alcohol $(165 \mathrm{mg}, 0.408 \mathrm{mmol})$ in THF (1.0
$\mathrm{mL})$ was added to a suspension of $\mathrm{KO}$ - $\mathrm{Bu}(95.0 \mathrm{mg}, 0.846$
$\mathrm{mmol}, 2.0$ equiv) in THF $(3.0 \mathrm{~mL})$ at $0{ }^{\circ} \mathrm{C}$. After stirred at 22 ${ }^{\circ} \mathrm{C}$ for $30 \mathrm{~min}$, the reaction mixture was recooled to $0{ }^{\circ} \mathrm{C}$, and $\mathrm{MPMCl}(85 \square \mathrm{L}, 0.63$ mmol, 1.5 equiv) was added. The mixture was warmed to $22{ }^{\circ} \mathrm{C}$ and stirred for an additional $24 \mathrm{~h}$. The mixture was diluted with saturated $\mathrm{NH}_{4} \mathrm{Cl}$ solution $(1 \mathrm{~mL})$ and water $(5 \mathrm{~mL})$. The mixture was extracted with EtOAc $(10 \mathrm{~mL} \times 3)$ and the combined organic layers were washed with brine $(10 \mathrm{~mL})$, dried, filtered, and concentrated to dryness. The residue was purified by silica gel chromatography (15:1 Hex:Et ${ }_{2} \mathrm{O}, \mathrm{R}_{f}=$ $0.10)$ to afford MPM ether $27(160 \mathrm{mg}, 0.305 \mathrm{mmol}, 75 \%)$ as a colorless oil. IR (neat): 2943 (m), 2865 (m), 1612 (m), 1513 (m), 1248 (m), 1091 (m), 821 (m), 685 (m). ${ }^{1} \mathrm{H}$ NMR (400 MHz, $\left.\mathrm{CDCl}_{3}\right)$ : $7.39(\mathrm{~d}, J=8.3 \mathrm{~Hz}, 2 \mathrm{H}), 7.33-7.19(\mathrm{~m}, 5 \mathrm{H}), 6.89-6.83$ (m, $2 \mathrm{H}), 6.57(\mathrm{~d}, J=15.9 \mathrm{~Hz}, 1 \mathrm{H}), 6.21(\mathrm{dd}, J=15.9,5.9 \mathrm{~Hz}, 1 \mathrm{H}), 4.47$ and $4.43(\mathrm{ABq}, J=$ $11.7 \mathrm{~Hz}$ ), 4.00-3.89 (m, 2H), 3.76 (s, 3H), 3.65 (ddd, $J=9.2,8.2,5.5 \mathrm{~Hz}, 1 \mathrm{H}), 3.62-3.53$ $(\mathrm{m}, 1 \mathrm{H}), 3.56(\mathrm{ddd}, J=9.2,5.7,5.7 \mathrm{~Hz}, 1 \mathrm{H}), 2.30-1.75(\mathrm{~m}, 4 \mathrm{H}), 1.42(\mathrm{ddd}, J=11.7$, 11.7, $11.7 \mathrm{~Hz}), 1.30(\mathrm{ddd}, J=11.7,11.7,11.7 \mathrm{~Hz}), 1.13-1.00(\mathrm{~m}, 21 \mathrm{H}) .{ }^{13} \mathrm{C} \mathrm{NMR}(100$ $\mathrm{MHz}_{\mathrm{CDCl}}$ ): $\square$ 138.46, 136.88, 130.80, 129.75, 128.65, 127.78, 126.65, 115.71, 75.91, 75.67, 69.40, 46.24, 45.83, 26.01. HRMS Calcd for $\mathrm{C}_{32} \mathrm{H}_{48} \mathrm{O}_{4} \mathrm{Si}:$ 524.3322. Found: 524.3324 .

-Proof of Stereochemistry: The absolute stereochemical identity of products obtained in this study have been determined by conversion of 4 a to known compound 28. ${ }^{2}$ The stereochemistry of other compounds has been determined by their conversion to 4 a. 
(2S,4R,6R)-Tetrahydro-2-hydroxymethyl-4-(triisopropylsilyloxy)-6-[2-(4methoxybenzyloxy)ethyl]-2H-pyran (28).

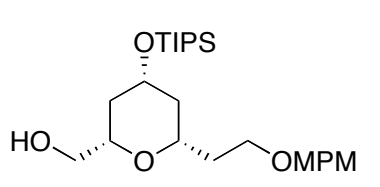

To a solution of olefin $27(20.2 \mathrm{mg}, 38.5 \square \mathrm{mol})$ in THF $(0.5 \mathrm{~mL})$ were added water $(0.5 \mathrm{~mL}), \mathrm{NaIO}_{4}(120 \mathrm{mg}, 0.561 \mathrm{mmol}, 15$ equiv), and a solution of $\mathrm{OsO}_{4}(2.5 \mathrm{wt} \%$ in $t-\mathrm{BuOH}, 20.0 \mathrm{mg}$ $1.97 \square \mathrm{mol})$ in $t$ - $\mathrm{BuOH}(0.5 \mathrm{~mL})$ at $0{ }^{\circ} \mathrm{C}$. The reaction mixture was stirred at $22{ }^{\circ} \mathrm{C}$ for $12 \mathrm{~h}$. The mixture was diluted with water $(3 \mathrm{~mL})$ and extracted with EtOAc $(5 \mathrm{~mL} \times 3)$. The combined organic layers were washed with brine $(5 \mathrm{~mL})$, dried, filtered, and concentrated to provide the crude aldehyde. A solution of $\mathrm{NaBH}_{4}(3.5$ $\mathrm{mg}, 93 \square \mathrm{mol})$ in methanol $(0.5 \mathrm{~mL})$ was added to a solution of the aldehyde in methanol $(0.5 \mathrm{~mL})$ at $0{ }^{\circ} \mathrm{C}$. After being stirred at $22{ }^{\circ} \mathrm{C}$ for $30 \mathrm{~min}$, the methanol was evaporated and the residue was diluted with water $(1 \mathrm{~mL})$ and extracted with EtOAc $(3 \mathrm{~mL} \times 3)$. The combined extracts were washed with brine, dried over $\mathrm{MgSO}_{4}$, filtered and concentrated to dryness. Silica gel chromatography of the residue (Hex:EtOAc 4:1, $\mathrm{R}_{f}=0.10$ ) afforded alcohol $28(10.1 \mathrm{mg}, 22.3 \mathrm{mmol}, 58 \%)$ as a colorless oil. [ []$^{25}{ }_{\mathrm{D}}-7.10^{\circ}(c 1.36$, $\mathrm{CHCl}_{3}$ ) [lit. ${ }^{2}[\square]^{22} \mathrm{D}-7.7^{\circ}\left(c\right.$ 1.32, $\left.\left.\mathrm{CHCl}_{3}\right)\right]$. IR (neat): 3458 (br), 2942 (s), 1514 (m), 1248 (m), 1093 (m), 883 (m), 820(m), 681 (m). ${ }^{1} \mathrm{H}$ NMR (400 MHz, CDCl $)$ ): 77.25 (d, $J=8.8 \mathrm{~Hz}, 2 \mathrm{H}), 6.88(\mathrm{~d}, J=8.8 \mathrm{~Hz}, 1 \mathrm{H}), 4.45$ and $4.41(\mathrm{ABq}, J=11.7 \mathrm{~Hz}, 2 \mathrm{H}), 3.89$ (dddd, $J=10.8,10.8,4.9,4.9 \mathrm{~Hz}, 1 \mathrm{H}), 3.80(\mathrm{~s}, 3 \mathrm{H}), 3.63-3.38(\mathrm{~m}, 6 \mathrm{H}), 2.00-1.71(\mathrm{~m}$, 4H), 1.95 (br, 1H), 1.34-1.17 (m, 2H), 1.10-0.95 (m, 21H). ${ }^{13} \mathrm{C}$ NMR (100 MHz, $\left.\mathrm{CDCl}_{3}\right): \square 159.34,130.71,129.40,113.96,76.08,72.87,72.80,68.61,66.42,66.28$, 55.43, 42.20, 37.71, 36.37, 18.22, 12.47. HRMS Calcd for $\mathrm{C}_{25} \mathrm{H}_{44} \mathrm{O}_{5} \mathrm{Si}: 452.2958$. Found: 452.2958.

General procedure for reductive ring-opening of pyran. A 2-neck $25 \mathrm{~mL}$ round bottom flask equipped with a Dewar condenser was cooled to $-78{ }^{\circ} \mathrm{C}$. UHP ammonia ( 3 $\mathrm{mL}$ ) was condensed into the flask. Sodium $(22.0 \mathrm{mg}, 0.957 \mathrm{mmol})$ was added under a stream of nitrogen; the ammonia solution immediately turns deep blue, stirring was continued until all of the sodium dissolved. Substrate $\mathbf{3 3}(6.1 \mathrm{mg}, 18 \square \mathrm{mol})$ was added by cannula as a solution in $\mathrm{Et}_{2} \mathrm{O}(0.3 \mathrm{~mL})$ and $t-\mathrm{BuOH}(77.5 \mathrm{mg}, 1.05 \mathrm{mmol})$. The reaction was quenched after one minute by adding solid $\mathrm{NH}_{4} \mathrm{Cl}$ until the blue color disappears; 5 $\mathrm{mL}$ of $\mathrm{CH}_{2} \mathrm{Cl}_{2}$ was then added and the ammonia was allowed to evaporate. The reaction was diluted with $10 \mathrm{~mL}$ of $\mathrm{H}_{2} \mathrm{O}$ and extracted with $10 \mathrm{~mL}$ portions of $\mathrm{CH}_{2} \mathrm{Cl}_{2}$ until no diol remained in the aqueous layer by TLC. The combined organic layers were dried over

(2) Paterson, I.; Tudge, M. Tetrahedron 2003, 59, 6833-6849. 
$\mathrm{MgSO}_{4}$, filtered, and the solvent removed in vacuo to deliver pure 34 (3.5 $\mathrm{mg}, 78 \%$ ) as a clear colourless oil.

\section{(2R,4S,6R)-2-Phenyl-6-(4-phenylbutyl)-1,3-dioxane-4-ethanol (29).}

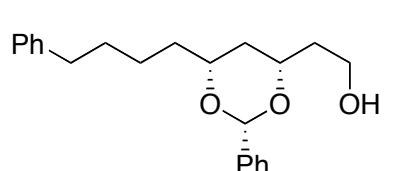

$\overline{\bar{P} h}$
General procedure for reductive ring-opening of pyran was

followed using 12 or $\mathbf{1 3}$ to yield a mixture of three products $(E / Z$ isomers and over-reduction). It is imperative that a reaction time

of one minute be employed to maximize the amount of desired product in the mixture: longer reaction times lead to a significant amount of reduction of the disubstituted olefin. This mixture was used without purification in the next step. A $25 \mathrm{~mL}$ flask was charged with the crude diol (214 mg, $0.921 \mathrm{mmol}$ ), benzaldehyde dimethylacetal (317 mg, 2.08 mmol), and a catalytic amount of $p$-toluenesulfonic acid $(\sim 10 \mathrm{mg})$. The flask was sparged with $\mathrm{N}_{2}$ and benzene $(8.0 \mathrm{~mL})$ was added by syringe; the reaction was then allowed to stir for $1 \mathrm{~h}$. The solvent was removed in vacuo and the reaction mixture was loaded directly onto a column of silica gel and eluted $\left(24: 1 \mathrm{Hex}_{\mathrm{Et}} \mathrm{O}\right)$ to deliver a mixture of three inseparable products ostensibly assigned to be $E / Z$ isomers and overreduction at the disubstituted olefin site (7:2:1 E:Z:Saturated). The mixture was used without further purification in the next reaction (The mixture is inconsequential in the context of this formal synthesis as the three products coalesce to a single compound after the hydrogenation step).

9-BBN (275 mg, $1.13 \mathrm{mmol})$ was dissolved in THF (4.5 mL) and added by cannula to a $25 \mathrm{~mL}$ flask containing a solution of the mixture of benzylidene acetals (see above) (270 $\mathrm{mg}, 0.842 \mathrm{mmol})$ in $\mathrm{THF}(4.5 \mathrm{~mL})$ at $22{ }^{\circ} \mathrm{C}$, and the mixture was stirred for $1 \mathrm{~h}$. The reaction was cooled to $0{ }^{\circ} \mathrm{C}$ before adding $\mathrm{pH} 7$ buffer $(1.5 \mathrm{~mL}, 0.05 \mathrm{M})$ and $30 \% \mathrm{H}_{2} \mathrm{O}_{2(\mathrm{aq})}(1.5 \mathrm{~mL})$; the mixture was then allowed to warm to $22{ }^{\circ} \mathrm{C}$ and stirred for an additional $12 \mathrm{~h}$. The reaction mixture was diluted with $\mathrm{H}_{2} \mathrm{O}(30 \mathrm{~mL})$ and extracted with $\mathrm{CH}_{2} \mathrm{Cl}_{2}(4 \times 30 \mathrm{~mL})$. The combined organic extracts were washed with brine, dried over $\mathrm{MgSO}_{4}$, filtered, and the solvent was removed in vacuo. The crude mixture was purified by silica gel chromatography using gradient elution $\left(4: 1 \square 2: 1 \mathrm{Hex}_{\mathrm{Et}} \mathrm{O}\right)$ to deliver an inseparable mixture of three primary alcohols $\left(192 \mathrm{mg}, \mathrm{R}_{f}=0.2,67 \%\right)$. The mixture of alcohols $(87.0 \mathrm{mg}, 0.257 \mathrm{mmol}$ ) was transferred to a $10 \mathrm{~mL}$ flask and dissolved in absolute EtOH. $10 \% \mathrm{Pt}(\mathrm{C})(8 \mathrm{mg})$ was added and the flask was sparged with $\mathrm{UHP}_{2}$ and then equipped with a balloon of $\mathrm{H}_{2}$. The mixture was stirred for $1 \mathrm{~h}$ at $22{ }^{\circ} \mathrm{C}$ and then filtered through celite with $\mathrm{Et}_{2} \mathrm{O}$ washings. The solvent was removed in vacuo to deliver pure 29 (86 mg, $0.25 \mathrm{mmol}, 98 \%$ ). IR (neat): 3415 (br), 3024 (w), 2924 (s), 2848 (m), 1451 (m), 1344 (m), 1130 (m), 1048 (m), 1023 (s), 752 (m), 702 (s). ${ }^{1} \mathrm{H}$ NMR (400 MHz, 
$\left.\mathrm{CDCl}_{3}\right)$ : $\square 7.48-7.46(\mathrm{~m}, 2 \mathrm{H}), 7.36-7.33(\mathrm{~m}, 3 \mathrm{H}), 7.29-7.26(\mathrm{~m}, 2 \mathrm{H}), 7.20-7.17(\mathrm{~m}, 3 \mathrm{H})$, $5.54(\mathrm{~s}, 1 \mathrm{H}), 4.08$ (dddd, $J=11.1,8.4,3.7,3.7 \mathrm{~Hz}, 1 \mathrm{H}), 3.85-3.80(\mathrm{~m}, 3 \mathrm{H}), 2.63$ (t, $J=$ $7.6 \mathrm{~Hz}, 2 \mathrm{H}), 2.14$ (br, 1H), 1.91-1.82 (m, 2H), 1.74-1.43 (m, 10H). ${ }^{13} \mathrm{C}$ NMR (100 MHz,

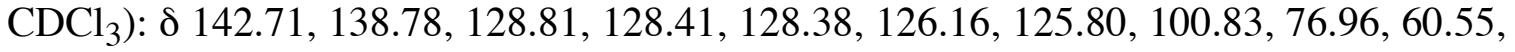
38.21, 37.01, 35.97, 35.84, 31.54, 24.83. HRMS Calcd for $\mathrm{C}_{22} \mathrm{H}_{28} \mathrm{O}_{3} \mathrm{Na}$ : 363.1936. Found: 363.1935 .

\section{(2R,4R,6R)-2-Phenyl-6-(4-phenylbutyl)-1,3-dioxane-4-acetaldehyde.}

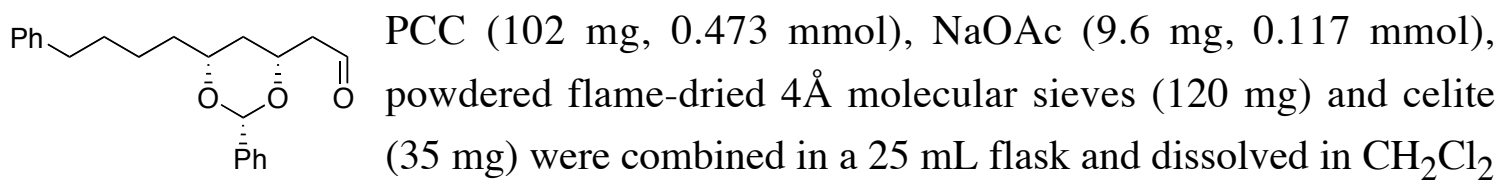
(1.0 mL). Alcohol 29 is added quantitatively by cannula using $4.0 \mathrm{~mL}$ of $\mathrm{CH}_{2} \mathrm{Cl}_{2}$. The reaction was allowed to stir for $2 \mathrm{~h}$ at $22{ }^{\circ} \mathrm{C}$ at which point $\mathrm{Et}_{2} \mathrm{O}(5.0 \mathrm{~mL})$ was added, to precipitate chromium salts, and the mixture was stirred for an additional $10 \mathrm{~min}$. The resulting mixture was filtered through a column ( 1 "W) containing one inch of celite (top layer) and one inch of silica gel (bottom layer). The aldehyde obtained was of sufficient purity (as judged by ${ }^{1} \mathrm{H}$ NMR analysis) for subsequent use. IR (neat): 3018 (w), 2924 (s), 2855 (m), 1728 (s), 1451 (m), 1344 (m), 1123 (m), 1029 (s), 759 (m), 696 (s). ${ }^{1} \mathrm{H}$ NMR $\left(400 \mathrm{MHz} \mathrm{CDCl}_{3}\right): \square 9.86(\mathrm{t}, J=1.9 \mathrm{~Hz}, 1 \mathrm{H}), 7.48-7.46(\mathrm{~m}, 2 \mathrm{H}), 7.36-7.34(\mathrm{~m}, 3 \mathrm{H})$, 7.28-7.26 (m, 2H), 7.18-7.16 (m, 3H), $5.60(\mathrm{~s}, 1 \mathrm{H}), 4.41-4.38(\mathrm{~m}, 1 \mathrm{H}), 3.87-3.84(\mathrm{~m}$, 1H), 2.84-2.78 (ddd, $J=16.9,7.3,2.0 \mathrm{~Hz}, 1 \mathrm{H}), 2.65-2.58$ (m, 3H), 1.74-1.42 (m, 8H). ${ }^{13} \mathrm{C} \mathrm{NMR}\left(100 \mathrm{MHz}, \mathrm{CDCl}_{3}\right)$ : $\square 200.64,142.67,138.49,128.89,128.54,128.42,128.35$, $126.17,125.82$, 100.83, 76.77, 72.04, 49.59, 36.81, 35.96, 35.78, 31.53, 24.80.

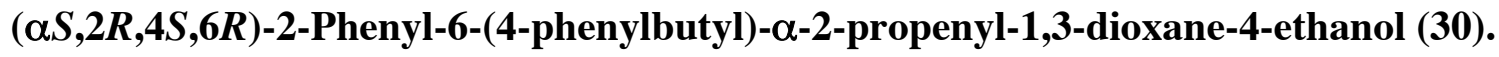

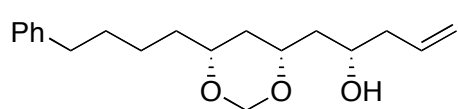

$\overline{\mathrm{Ph}}$
$\square-(-)$-Methoxy isopinocampheylborane $(8.0 \mathrm{mg}, 25.3 \square \mathrm{mol})$ was dissolved in $0.25 \mathrm{~mL}$ of $\mathrm{Et}_{2} \mathrm{O}$ and cooled to $0{ }^{\circ} \mathrm{C}$. A solution of allyl magnesium bromide (21.3 $\square \mathrm{L}, 0.95 \mathrm{M}, 20.2$

$\square \mathrm{mol}$ ) was added by syringe and the resulting solution was stirred for $1 \mathrm{~h}$. The solution was then cooled to $-78{ }^{\circ} \mathrm{C}$ and the aldehyde $(3.0 \mathrm{mg}, 8.9 \square \mathrm{mol})$ was added dropwise by syringe. The mixture was allowed to stir at $-78{ }^{\circ} \mathrm{C}$ for $2 \mathrm{~h}$ and then warmed to $0{ }^{\circ} \mathrm{C}$. One drop each of $30 \% \mathrm{H}_{2} \mathrm{O}_{2(\mathrm{aq})}$ and $1 \mathrm{~N} \mathrm{NaOH}$ are added and the reaction was stirred at $22{ }^{\circ} \mathrm{C}$ for $3 \mathrm{~h}$. The reaction was diluted with $\mathrm{H}_{2} \mathrm{O}(1 \mathrm{~mL})$ and extracted with EtOAc $(3 \times 1 \mathrm{~mL})$. The combined organic layers were dried over $\mathrm{MgSO}_{4}$, filtered and the solvent removed in vacuo. The crude reaction mixture was further purified by silica gel chromatography $(9: 1$ hex: $\left.\mathrm{Et}_{2} \mathrm{O}, \mathrm{R}_{f}=0.2\right)$ to deliver 31 as a clear colourless oil $(2.8 \mathrm{mg}, 82 \%)$ as a $3.5: 1$ 
mixture of diastereomers. This compound was identical to that already reported ${ }^{3}$ as judged by ${ }^{1} \mathrm{H}$ and ${ }^{13} \mathrm{C}$ NMR.

\section{4-Benzyloxy-3,5-dimethyl-2-(E)-styryl-6-vinyltetrahydro-2H-pyran (33).}

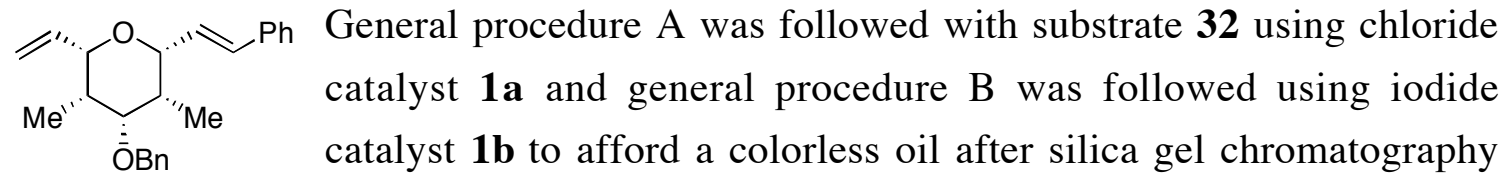

(19:1 4:1 Hex:Et $\left.{ }_{2} \mathrm{O}, \mathrm{R}_{f}=0.3\right)$. IR (neat): $3062(\mathrm{w}), 3024(\mathrm{w}), 2974(\mathrm{w}), 2886(\mathrm{~m})$, 2836 (w), 1495 (w), 1451 (m), 1357 (w), 1092 (s), 1080 (m), 960 (s), 752 (s), 683 (s). ${ }^{1} \mathrm{H}$ NMR (400 MHz, $\left.\mathrm{CDCl}_{3}\right)$ : $\square 7.42-7.35(\mathrm{~m}, 6 \mathrm{H}), 7.34-7.30(\mathrm{~m}, 3 \mathrm{H}), 7.25-7.21(\mathrm{~m}, 1 \mathrm{H})$, $6.67(\mathrm{~d}, J=16.1 \mathrm{~Hz}, 1 \mathrm{H}), 6.23$ $(\mathrm{dd}, J=16.1,5.4 \mathrm{~Hz}, 1 \mathrm{H})$, 5.90 (ddd, $J=17.3,10.7,5.1$ $\mathrm{Hz}, 1 \mathrm{H}), 5.38$ (ddd, $J=17.3$, $1.7,1.7 \mathrm{~Hz}, 1 \mathrm{H}), 5.20$ (ddd, $J$ $=10.7,1.6,1.6 \mathrm{~Hz}, 1 \mathrm{H}), 4.59$

$(\mathrm{s}, 2 \mathrm{H}), 4.17-4.15(\mathrm{~m}, 1 \mathrm{H})$, 4.05-4.03 (m, 1H), 3.75 (dd, $J$ $=10.5,10.5 \mathrm{~Hz}, 1 \mathrm{H})$, 2.24-2.13 (m, 2H), $1.03(\mathrm{~d}, J$ $=7.3 \mathrm{~Hz}, 3 \mathrm{H}), 1.01(\mathrm{~d}, J=7.3$ $\mathrm{Hz}, 3 \mathrm{H}) .{ }^{13} \mathrm{C}$ NMR (100 MHz,
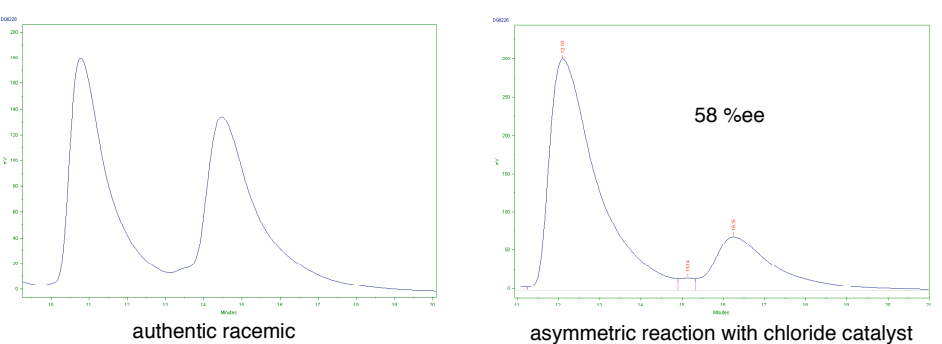

asymmetric reaction with chloride catalyst

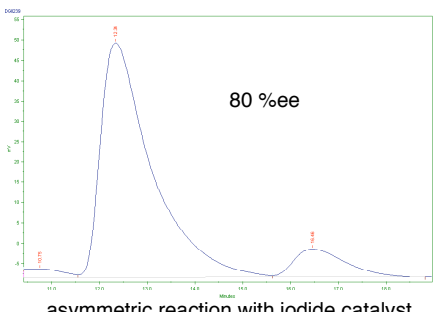

Conditions: 95:5 Hex:IPA, Chiracel AD, $1.0 \mathrm{~mL} / \mathrm{min}, 210 \mathrm{~nm}$ Retention Times: $12.1 \mathrm{~min}, 16.3 \mathrm{~min}$ $\left.\mathrm{CDCl}_{3}\right): \square 139.00,137.29,137.19,130.11,128.89,128.63,128.51,127.56,127.48$, $127.39,126.53,115.22,80.44,80.07,79.45,69.26,37.09,36.57,9.60,9.37$. HRMS Calcd for $\mathrm{C}_{24} \mathrm{H}_{28} \mathrm{O}_{2}$ : 348.2089. Found: No molecular ion detected.

The ee of $\mathbf{3 4}$ was determined by HPLC of diol 35, which is generated from 34 as described below.

(2S,3R,4S,5S)-4,6-Dimethyl-9-phenylnona-1,7-dien-3,5-diol (34).

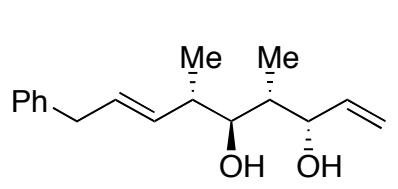

General procedure for reductive ring-opening of pyrans was followed with $\mathbf{3 3}$ to deliver pure $\mathbf{3 4}$ as a clear colourless oil. IR (neat): 3345 (br), 2968 (m), 2905 (s), 1451 (s), 1111 (w), 973 (s), 916 (m), 746 (m), 696 (s). ${ }^{1} \mathrm{H}$ NMR (400 MHz, $\left.\mathrm{CDCl}_{3}\right)$ : $\square$ 7.36-7.26 (m, 2H), 7.24-7.15 (m, 3H), 5.91 (ddd, $J=17.1,10.6,5.3 \mathrm{~Hz}, 1 \mathrm{H}), 5.72$ (ddd,

(3) Tosaki, S.; Nemoto, T.; Ohshima, T.; Shibasaki, M. Org. Lett. 2003, 5, 495-498. 
$J=15.3,6.8,6.8 \mathrm{~Hz}, 1 \mathrm{H}), 5.51(\mathrm{dd}, J=15.4,8.5 \mathrm{~Hz}, 1 \mathrm{H}), 5.30$ (ddd, $J=17.2,1.1,1.1$ $\mathrm{Hz}, 1 \mathrm{H}$ ), 5.19 (ddd, $J=10.6,1.2,1.2 \mathrm{~Hz}, 1 \mathrm{H}), 4.50$ (br s, 1H), 3.45-3.42 (m, 1H), 3.39 $(\mathrm{d}, J=6.8 \mathrm{~Hz}, 2 \mathrm{H}), 3.13(\mathrm{~d}, J=2.0 \mathrm{~Hz}, 1 \mathrm{H}), 2.47-2.38(\mathrm{~m}, 2 \mathrm{H}), 1.91-1.84(\mathrm{~m}, 1 \mathrm{H}), 1.06$ $(\mathrm{d}, J=6.9 \mathrm{~Hz}, 3 \mathrm{H}), 0.93(\mathrm{~d}, J=7.1 \mathrm{~Hz}, 3 \mathrm{H}) .{ }^{13} \mathrm{C} \mathrm{NMR}\left(100 \mathrm{MHz}, \mathrm{CDCl}_{3}\right): \square$ 140.62, $138.90,132.45,131.80,128.64,128.57,126.23,115.18,78.77,74.24,40.39,39.52$, 39.31, 17.71, 11.98. HRMS Calcd for $\mathrm{C}_{17} \mathrm{H}_{21} \mathrm{O}_{2}[\mathrm{M}-3 \mathrm{H}]:$ 257.1543. Found: [M-3H] 257.1542 .

Table S1: Recycling studies using Chloride Catalyst 1a

\begin{tabular}{|c|c|c|c|c|c|}
\hline & $\underset{=}{\mathrm{OH}}$ & $\begin{array}{l}5 \text { mol\% 1a } \\
\begin{array}{c}\text { Ph } \\
(2 \text { equiv })\end{array} \\
\text { THF, } 22^{\circ} \mathrm{C}\end{array}$ & Pn & $\stackrel{\mathrm{OH}}{\vdots}$ & \\
\hline Cycle & $\begin{array}{l}\text { time }(\mathrm{h}) \\
\text { conv }(\%)\end{array}$ & yield (\%) & $E: Z$ & ee $(\%)$ & $\begin{array}{l}\text { rec. cat. } \\
\text { yield (\%) }\end{array}$ \\
\hline Cycle 1 & $1 ;>98$ & 80 & $>98: 2$ & 94 & 90 \\
\hline Cycle 2 & $1 ;>98$ & 75 & $>98: 2$ & 93 & 85 \\
\hline Cycle 3 & $1 ;>98$ & 78 & $>98: 2$ & 94 & 88 \\
\hline Cycle 4 & $1.5 ;>98$ & 78 & $>98: 2$ & 94 & 80 \\
\hline Cycle 5 & $1.5 ;>98$ & 72 & $>98: 2$ & 94 & 67 \\
\hline
\end{tabular}

Table S2: Optimization of Allylation Reaction of Aldehyde 30.5

\begin{tabular}{|c|c|c|c|}
\hline $\mathrm{MX}_{2}$ & Temp & Solvent & d.r $(30: 30 b)$ \\
\hline $\mathrm{MgBr}_{2}$ & $0^{\circ} \mathrm{C}->\mathrm{rt}$ & $\mathrm{THF} \mathrm{Et}_{2} \mathrm{O}$ & $1.1: 1$ \\
\hline $\mathrm{MgBr}_{2}$ & $-78^{\circ} \mathrm{C}$ & $\mathrm{THF} / \mathrm{Et}_{2} \mathrm{O}$ & $1.3: 1$ \\
\hline$(\mathrm{R}, \mathrm{R})-\mathbf{3 5}$ & $-10^{\circ} \mathrm{C}$ & PhMe & $1: 5$ \\
\hline$(\mathrm{S}, \mathrm{S})-35$ & $-10^{\circ} \mathrm{C}$ & PhMe & $1: 1$ \\
\hline$(\mathrm{S}, \mathrm{S})-\mathbf{3 6}$ & $-18^{\circ} \mathrm{C}$ & $\mathrm{CH}_{2} \mathrm{Cl}_{2}$ & $1: 9$ \\
\hline$(\mathrm{R}, \mathrm{R})-\mathbf{3 6}$ & $-18^{\circ} \mathrm{C}$ & $\mathrm{CH}_{2} \mathrm{Cl}_{2}$ & $1: 1.2$ \\
\hline$(+) \mathrm{B}(\mathrm{lpc})_{2}$ & $-78^{\circ} \mathrm{C}$ & $\mathrm{Et}_{2} \mathrm{O}$ & $1: 2.5$ \\
\hline$(-) \mathrm{B}(\mathrm{Ipc})_{2}$ & $-78^{\circ} \mathrm{C}$ & $\mathrm{Et}_{2} \mathrm{O}$ & $3.5: 1$ \\
\hline
\end{tabular}

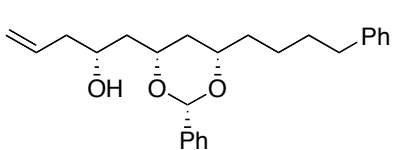

30
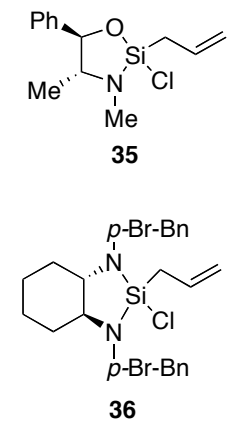

Preparation of reagents $3^{4}$ and $\mathbf{3 6}^{5}$ were completed as already reported with the exception that $\mathbf{3 6}$ was purified in a glovebox filled with $\mathrm{N}_{2}$.

(4) Kinnaird, J. W.; Ng, P. Y.; Kubota, K.; Wang, X.; Leighton, J. L. J. Am. Chem. Soc. 2002, 124, $7920-7921$.

(5) Kubota, K.; Leighton, J. L. Angew. Chem. Int. Ed. 2003, 42, 946-948. 


\section{General experimental for X-Ray analysis}

Data was collected using a Bruker APEX CCD (charged coupled deviced) based diffractometer equipped with an LT-2 low temperature apparatus operating at $193 \mathrm{~K}$. A suitable crystal was chosen and mounted on a glass fiber using grease. Data was measured using omega scans of $0.3^{\circ}$ per frame for 30 seconds, such that a hemisphere was collected. A total of 1305 frames were collected with a maximum resolution of 0.90 $\AA$. Cell parameters were retrieved using $\mathrm{SMART}^{6}$ software and refined using SAINT on all observed reflections. Data reduction was performed using the SAINT software ${ }^{7}$, which corrects for Lp and decay. Absorption corrections were applied using SADABS supplied by George Sheldrick. The structures ware solved by the direct method using the SHELXS-978 program and refined by least squares method on $\mathrm{F}^{2}$, SHELXL-979 incorporated in SHELXTL-PC V 6.10 ${ }^{10}$.

All non-hydrogen atoms are refined anisotropically. Hydrogens were calculated by geometrical methods and refined as a riding model. The crystal used for the diffraction study showed no decomposition during data collection. All drawings are done at $30 \%$ ellipsoids.

\section{X-Ray Data for Iodide Complex $1 b$}

\section{Table 1. Crystal data and structure refinement for DG01t}

Identification code

Empirical formula

Formula weight

Temperature

Wavelength

Crystal system

Space group

Unit cell dimensions $\operatorname{dg} 01 \mathrm{t}$

C48 H43 I N2 O2 Ru

907.81

193(2) K

$0.71073 \approx$

Monoclinic

P21

$$
\mathrm{a}=11.6174(7) \approx \quad \mathrm{a}=90 \infty \text {. }
$$

(6) SMART V5.626 (NT) Software for the CCD Detector Systems; Bruker Analytical X-ray Systems, Madison, WI (2001)

(7) SAINT V 5.01 (NT) Software for the CCD Detector Systems; Bruker Analytical X-ray Systems, Madison, WI (2001)

(8) Sheldrick, G. M. SHELXS-90, Program for the Solution of Crystal Structure, University of Göttingen, Germany, 1990

(9) Sheldrick, G. M. SHELXL-97, Program for the Refinement of Crystal Structure, University of Göttingen, Germany, 1997.

(10) SHELXTL 6.0 (PC-Version), Program Library for Structure Solution and Molecular Graphics; Bruker Analytical X-ray Systems, Madison, WI (1998) 
Volume

Z

Density (calculated)

Absorption coefficient

$\mathrm{F}(000)$

Crystal size

Theta range for data collection

Index ranges

Reflections collected

Independent reflections

Completeness to theta $=28.29 \circ 0$

Refinement method

Data / restraints / parameters

Goodness-of-fit on $\mathrm{F}^{2}$

Final $R$ indices [I $>2 \operatorname{sigma}(\mathrm{I})]$

$\mathrm{R}$ indices (all data)

Absolute structure parameter

Largest diff. peak and hole $\mathrm{b}=9.8883(6) \approx$ $\mathrm{b}=92.5470(10) \infty$.

$\mathrm{c}=17.5221(11) \approx$ $\mathrm{g}=90 \infty$.

$$
\text { 2010.9(2) } \approx^{3}
$$

2

$1.499 \mathrm{Mg} / \mathrm{m}^{3}$

$1.199 \mathrm{~mm}^{-1}$

916

$0.5 \times 0.5 \times 0.5 \mathrm{~mm}^{3}$

1.16 to $28.29 \infty$.

$-11<=\mathrm{h}<=15,-11<=\mathrm{k}<=13,-20<=1<=23$

15089

$9365[\mathrm{R}($ int $)=0.0142]$

$99.6 \%$

Full-matrix least-squares on $\mathrm{F}^{2}$

9365 / 1 / 492

1.014

$\mathrm{R} 1=0.0219, \mathrm{wR} 2=0.0533$

$\mathrm{R} 1=0.0227, \mathrm{wR} 2=0.0537$

$-0.015(8)$

0.791 and -0.230 e. $\sim^{-3}$

Table 2. Atomic coordinates $\left(\times 10^{4}\right)$ and equivalent isotropic displacement parameters $\left(\approx^{2} \times 10^{3}\right)$ for DG01t.

$\mathrm{U}(\mathrm{eq})$ is defined as one third of the trace of the orthogonalized $\mathrm{Uij}^{\mathrm{ij}}$ tensor.

\begin{tabular}{lcccc}
\hline & $\mathrm{x}$ & $\mathrm{y}$ & $\mathrm{z}$ & $\mathrm{U}(\mathrm{eq})$ \\
\hline $\mathrm{Ru}(1)$ & & & \\
$\mathrm{I}(2)$ & $2793(1)$ & $6397(1)$ & $7634(1)$ & $23(1)$ \\
$\mathrm{O}(4)$ & $4673(1)$ & $4898(1)$ & $7879(1)$ & $36(1)$ \\
$\mathrm{C}(5)$ & $4003(1)$ & $8151(2)$ & $7877(1)$ & $26(1)$ \\
$\mathrm{C}(6)$ & $-142(2)$ & $8112(2)$ & $6787(1)$ & $30(1)$ \\
$\mathrm{C}(7)$ & $2394(2)$ & $4870(3)$ & $6100(1)$ & $24(1)$ \\
$\mathrm{C}(8)$ & $2421(2)$ & $6433(3)$ & $5042(1)$ & $24(1)$ \\
$\mathrm{C}(54)$ & $776(2)$ & $6521(2)$ & $5948(1)$ & $24(1)$ \\
$\mathrm{C}(9)$ & $2430(2)$ & $6792(2)$ & $8618(1)$ & $29(1)$ \\
& $-299(2)$ & $6333(2)$ & $5543(1)$ & $24(1)$
\end{tabular}




\begin{tabular}{|c|c|c|c|c|}
\hline$C(10)$ & $1867(2)$ & $5894(2)$ & 5691(1) & $24(1)$ \\
\hline $\mathrm{C}(11)$ & $-437(2)$ & $5444(2)$ & $4906(1)$ & $28(1)$ \\
\hline$C(12)$ & $4046(2)$ & $4906(3)$ & $5314(1)$ & $32(1)$ \\
\hline $\mathrm{C}(13)$ & $5788(2)$ & $9894(3)$ & $8689(1)$ & $29(1)$ \\
\hline$C(14)$ & $3955(2)$ & $8510(2)$ & $8645(1)$ & $25(1)$ \\
\hline$C(15)$ & $-2461(2)$ & $5945(3)$ & $4783(2)$ & $39(1)$ \\
\hline$C(16)$ & $-272(2)$ & 4929(3) & $8708(1)$ & $38(1)$ \\
\hline$C(17)$ & $3587(2)$ & $7533(3)$ & $3813(1)$ & $40(1)$ \\
\hline$C(18)$ & $1917(2)$ & $7456(2)$ & $4573(1)$ & $30(1)$ \\
\hline$C(19)$ & $4092(2)$ & $6546(3)$ & $4244(1)$ & $35(1)$ \\
\hline$C(20)$ & $2914(2)$ & $8149(3)$ & $9788(1)$ & $33(1)$ \\
\hline $\mathrm{C}(21)$ & $3490(2)$ & $4356(2)$ & 5911(1) & $30(1)$ \\
\hline$C(22)$ & $3076(2)$ & $7838(2)$ & $9018(1)$ & $27(1)$ \\
\hline$C(23)$ & $-1293(2)$ & $7043(2)$ & $5775(1)$ & $29(1)$ \\
\hline$C(24)$ & $-1173(2)$ & $7964(3)$ & $6397(1)$ & $33(1)$ \\
\hline$C(25)$ & $3535(2)$ & $5944(2)$ & $4863(1)$ & $28(1)$ \\
\hline $\mathrm{N}(2)$ & $1109(2)$ & $4033(2)$ & $7836(1)$ & $35(1)$ \\
\hline $\mathrm{C}(27)$ & $15(2)$ & $4631(3)$ & $10074(2)$ & $45(1)$ \\
\hline $\mathrm{C}(28)$ & $756(2)$ & $4236(3)$ & $8607(1)$ & $33(1)$ \\
\hline C(29) & $858(2)$ & $7378(2)$ & $6578(1)$ & $25(1)$ \\
\hline $\mathrm{C}(30)$ & $4722(2)$ & $9385(2)$ & $9028(1)$ & $28(1)$ \\
\hline $\mathrm{C}(31)$ & $2479(2)$ & 7999(3) & $3974(1)$ & $37(1)$ \\
\hline $\mathrm{C}(32)$ & $6596(2)$ & $8958(3)$ & $8443(1)$ & $36(1)$ \\
\hline C(33) & $-1492(2)$ & $5259(2)$ & $4541(1)$ & $35(1)$ \\
\hline$C(34)$ & $4291(2)$ & $9173(2)$ & $7300(1)$ & $29(1)$ \\
\hline$C(35)$ & $-2367(2)$ & $6824(3)$ & $5386(1)$ & $36(1)$ \\
\hline$C(36)$ & $1402(2)$ & $3689(3)$ & $9215(1)$ & $38(1)$ \\
\hline $\mathrm{C}(37)$ & $3407(2)$ & 10294(3) & $7286(1)$ & $40(1)$ \\
\hline $\mathrm{C}(38)$ & $5999(2)$ & $11265(3)$ & $8600(1)$ & $41(1)$ \\
\hline C(39) & $6966(3)$ & $11693(3)$ & $8239(2)$ & $52(1)$ \\
\hline $\mathrm{C}(40)$ & $7567(2)$ & $9402(3)$ & $8090(2)$ & $46(1)$ \\
\hline$C(41)$ & $1009(2)$ & $3915(3)$ & 9952(1) & $44(1)$ \\
\hline$C(42)$ & $3601(2)$ & $9109(3)$ & $10159(1)$ & $37(1)$ \\
\hline$C(43)$ & $4504(2)$ & $9703(3)$ & $9789(1)$ & $34(1)$ \\
\hline $\mathrm{C}(44)$ & $7747(3)$ & $10763(4)$ & $7984(2)$ & $53(1)$ \\
\hline$C(45)$ & $-617(2)$ & $5133(3)$ & $9454(2)$ & $43(1)$ \\
\hline
\end{tabular}




\begin{tabular}{lrrrr}
$\mathrm{O}(1)$ & $1855(1)$ & $7638(2)$ & $6957(1)$ & $27(1)$ \\
$\mathrm{N}(1)$ & $1832(2)$ & $4257(2)$ & $6723(1)$ & $26(1)$ \\
$\mathrm{C}(55)$ & $1818(2)$ & $4811(2)$ & $7433(1)$ & $26(1)$ \\
$\mathrm{C}(50)$ & $4382(2)$ & $8396(3)$ & $6556(1)$ & $36(1)$ \\
$\mathrm{C}(49)$ & $-373(3)$ & $4868(5)$ & $10875(2)$ & $66(1)$ \\
$\mathrm{C}(52)$ & $991(3)$ & $3183(3)$ & $6592(1)$ & $46(1)$ \\
$\mathrm{C}(51)$ & $652(2)$ & $2866(3)$ & $7403(1)$ & $41(1)$ \\
$\mathrm{C}(48)$ & $2506(3)$ & $2924(4)$ & $9116(2)$ & $52(1)$ \\
$\mathrm{C}(47)$ & $-996(3)$ & $5485(4)$ & $8040(2)$ & $57(1)$ \\
\hline
\end{tabular}

\section{Table 3. Bond lengths $[\approx]$ and angles $[\infty]$ for DG01t.}

$\begin{array}{ll}\mathrm{Ru}(1)-\mathrm{C}(54) & 1.835(2) \\ \mathrm{Ru}(1)-\mathrm{C}(55) & 1.958(2) \\ \mathrm{Ru}(1)-\mathrm{O}(1) & 1.9974(15) \\ \mathrm{Ru}(1)-\mathrm{O}(4) & 2.2603(15) \\ \mathrm{Ru}(1)-\mathrm{I}(2) & 2.6583(2) \\ \mathrm{O}(4)-\mathrm{C}(14) & 1.395(2) \\ \mathrm{O}(4)-\mathrm{C}(34) & 1.479(3) \\ \mathrm{C}(5)-\mathrm{C}(24) & 1.360(3) \\ \mathrm{C}(5)-\mathrm{C}(29) & 1.432(3) \\ \mathrm{C}(5)-\mathrm{H}(5) & 0.9500 \\ \mathrm{C}(6)-\mathrm{C}(10) & 1.369(3) \\ \mathrm{C}(6)-\mathrm{C}(21) & 1.424(3) \\ \mathrm{C}(6)-\mathrm{N}(1) & 1.432(3) \\ \mathrm{C}(7)-\mathrm{C}(18) & 1.414(3) \\ \mathrm{C}(7)-\mathrm{C}(25) & 1.429(3) \\ \mathrm{C}(7)-\mathrm{C}(10) & 1.434(3) \\ \mathrm{C}(8)-\mathrm{C}(29) & 1.392(3) \\ \mathrm{C}(8)-\mathrm{C}(9) & 1.421(3) \\ \mathrm{C}(8)-\mathrm{C}(10) & 1.498(3) \\ \mathrm{C}(54)-\mathrm{C}(22) & 1.441(3) \\ \mathrm{C}(54)-\mathrm{H}(54) & 0.9500 \\ \mathrm{C}(9)-\mathrm{C}(11) & 1.423(3) \\ \mathrm{C}(9)-\mathrm{C}(23) & 1.427(3) \\ \mathrm{C}(11)-\mathrm{C}(33) & 1.370(3) \\ & \end{array}$




$\begin{array}{ll}\mathrm{C}(11)-\mathrm{H}(11) & 0.9500 \\ \mathrm{C}(12)-\mathrm{C}(21) & 1.366(3) \\ \mathrm{C}(12)-\mathrm{C}(25) & 1.409(3) \\ \mathrm{C}(12)-\mathrm{H}(12) & 0.9500 \\ \mathrm{C}(13)-\mathrm{C}(38) & 1.388(4) \\ \mathrm{C}(13)-\mathrm{C}(32) & 1.400(3) \\ \mathrm{C}(13)-\mathrm{C}(30) & 1.485(3) \\ \mathrm{C}(14)-\mathrm{C}(30) & 1.392(3) \\ \mathrm{C}(14)-\mathrm{C}(22) & 1.404(3) \\ \mathrm{C}(15)-\mathrm{C}(35) & 1.369(4) \\ \mathrm{C}(15)-\mathrm{C}(33) & 1.396(4) \\ \mathrm{C}(15)-\mathrm{H}(15) & 0.9500 \\ \mathrm{C}(16)-\mathrm{C}(28) & 1.395(4) \\ \mathrm{C}(16)-\mathrm{C}(45) & 1.399(3) \\ \mathrm{C}(16)-\mathrm{C}(47) & 1.514(4) \\ \mathrm{C}(17)-\mathrm{C}(19) & 1.352(4) \\ \mathrm{C}(17)-\mathrm{C}(31) & 1.407(4) \\ \mathrm{C}(17)-\mathrm{H}(17) & 0.9500 \\ \mathrm{C}(18)-\mathrm{C}(31) & 1.370(3) \\ \mathrm{C}(18)-\mathrm{H}(18) & 0.9500 \\ \mathrm{C}(19)-\mathrm{C}(25) & 1.419(3) \\ \mathrm{C}(19)-\mathrm{H}(19) & 0.9500 \\ \mathrm{C}(20)-\mathrm{C}(42) & 1.384(4) \\ \mathrm{C}(20)-\mathrm{C}(22) & 1.406(3) \\ \mathrm{C}(20)-\mathrm{H}(20) & 0.9500 \\ \mathrm{C}(21)-\mathrm{H}(21) & 0.9500 \\ \mathrm{C}(23)-\mathrm{C}(35) & 1.412(3) \\ \mathrm{C}(23)-\mathrm{C}(24) & 1.422(3) \\ \mathrm{C}(24)-\mathrm{H}(24) & 0.9500 \\ \mathrm{~N}(2)-\mathrm{C}(55) & 1.349(3) \\ \mathrm{N}(2)-\mathrm{C}(28) & 1.443(3) \\ \mathrm{N}(2)-\mathrm{C}(51) & 1.468(3) \\ \mathrm{C}(27)-\mathrm{C}(41) & 1.380(4) \\ \mathrm{C}(27)-\mathrm{C}(45) & 1.377(4) \\ \mathrm{C}(27)-\mathrm{C}(49) & 1.511(4) \\ \mathrm{C}(28)-\mathrm{C}(36) & 1.385(4) \\ & \\ & \end{array}$




$\begin{array}{ll}\mathrm{C}(29)-\mathrm{O}(1) & 1.334(2) \\ \mathrm{C}(30)-\mathrm{C}(43) & 1.404(3) \\ \mathrm{C}(31)-\mathrm{H}(31) & 0.9500 \\ \mathrm{C}(32)-\mathrm{C}(40) & 1.381(4) \\ \mathrm{C}(32)-\mathrm{H}(32) & 0.9500 \\ \mathrm{C}(33)-\mathrm{H}(33) & 0.9500 \\ \mathrm{C}(34)-\mathrm{C}(37) & 1.511(4) \\ \mathrm{C}(34)-\mathrm{C}(50) & 1.521(3) \\ \mathrm{C}(34)-\mathrm{H}(34) & 1.0000 \\ \mathrm{C}(35)-\mathrm{H}(35) & 0.9500 \\ \mathrm{C}(36)-\mathrm{C}(41) & 1.407(3) \\ \mathrm{C}(36)-\mathrm{C}(48) & 1.505(4) \\ \mathrm{C}(37)-\mathrm{H}(37 \mathrm{~A}) & 0.9800 \\ \mathrm{C}(37)-\mathrm{H}(37 \mathrm{~B}) & 0.9800 \\ \mathrm{C}(37)-\mathrm{H}(37 \mathrm{C}) & 0.9800 \\ \mathrm{C}(38)-\mathrm{C}(39) & 1.379(4) \\ \mathrm{C}(38)-\mathrm{H}(38) & 0.9500 \\ \mathrm{C}(39)-\mathrm{C}(44) & 1.381(5) \\ \mathrm{C}(39)-\mathrm{H}(39) & 0.9500 \\ \mathrm{C}(40)-\mathrm{C}(44) & 1.376(5) \\ \mathrm{C}(40)-\mathrm{H}(40) & 0.9500 \\ \mathrm{C}(41)-\mathrm{H}(41) & 0.9500 \\ \mathrm{C}(42)-\mathrm{C}(43) & 1.388(3) \\ \mathrm{C}(42)-\mathrm{H}(42) & 0.9500 \\ \mathrm{C}(43)-\mathrm{H}(43) & 0.9500 \\ \mathrm{C}(44)-\mathrm{H}(44) & 0.9500 \\ \mathrm{C}(45)-\mathrm{H}(45) & 0.9500 \\ \mathrm{~N}(1)-\mathrm{C}(55) & 1.360(3) \\ \mathrm{N}(1)-\mathrm{C}(52) & 1.454(3) \\ \mathrm{C}(50)-\mathrm{H}(50 \mathrm{~A}) & 0.9800 \\ \mathrm{C}(50)-\mathrm{H}(50 \mathrm{~B}) & 0.9800 \\ \mathrm{C}(50)-\mathrm{H}(50 \mathrm{C}) & 0.9800 \\ \mathrm{C}(49)-\mathrm{H}(49 \mathrm{~A}) & 0.9800 \\ \mathrm{C}(49)-\mathrm{H}(49 \mathrm{~B}) & 0.9800 \\ \mathrm{C}(49)-\mathrm{H}(49 \mathrm{C}) & 0.9800 \\ \mathrm{C}(52)-\mathrm{C}(51) & 1.523(3) \\ & \end{array}$




$\begin{array}{lc}\mathrm{C}(52)-\mathrm{H}(52 \mathrm{~A}) & 0.9900 \\ \mathrm{C}(52)-\mathrm{H}(52 \mathrm{~B}) & 0.9900 \\ \mathrm{C}(51)-\mathrm{H}(51 \mathrm{~A}) & 0.9900 \\ \mathrm{C}(51)-\mathrm{H}(51 \mathrm{~B}) & 0.9900 \\ \mathrm{C}(48)-\mathrm{H}(48 \mathrm{~A}) & 0.9800 \\ \mathrm{C}(48)-\mathrm{H}(48 \mathrm{~B}) & 0.9800 \\ \mathrm{C}(48)-\mathrm{H}(48 \mathrm{C}) & 0.9800 \\ \mathrm{C}(47)-\mathrm{H}(47 \mathrm{~A}) & 0.9800 \\ \mathrm{C}(47)-\mathrm{H}(47 \mathrm{~B}) & 0.9800 \\ \mathrm{C}(47)-\mathrm{H}(47 \mathrm{C}) & 0.9800 \\ & \\ \mathrm{C}(54)-\mathrm{Ru}(1)-\mathrm{C}(55) & 100.60(9) \\ \mathrm{C}(54)-\mathrm{Ru}(1)-\mathrm{O}(1) & 106.61(8) \\ \mathrm{C}(55)-\mathrm{Ru}(1)-\mathrm{O}(1) & 95.10(7) \\ \mathrm{C}(54)-\mathrm{Ru}(1)-\mathrm{O}(4) & 80.04(8) \\ \mathrm{C}(55)-\mathrm{Ru}(1)-\mathrm{O}(4) & 176.85(8) \\ \mathrm{O}(1)-\mathrm{Ru}(1)-\mathrm{O}(4) & 87.65(6) \\ \mathrm{C}(54)-\mathrm{Ru}(1)-\mathrm{I}(2) & 100.84(7) \\ \mathrm{C}(55)-\mathrm{Ru}(1)-\mathrm{I}(2) & 92.70(6) \\ \mathrm{O}(1)-\mathrm{Ru}(1)-\mathrm{I}(2) & 149.48(4) \\ \mathrm{O}(4)-\mathrm{Ru}(1)-\mathrm{I}(2) & 84.15(4) \\ \mathrm{C}(14)-\mathrm{O}(4)-\mathrm{C}(34) & 120.37(16) \\ \mathrm{C}(14)-\mathrm{O}(4)-\mathrm{Ru}(1) & 109.03(12) \\ \mathrm{C}(34)-\mathrm{O}(4)-\mathrm{Ru}(1) & 123.61(12) \\ \mathrm{C}(24)-\mathrm{C}(5)-\mathrm{C}(29) & 121.5(2) \\ \mathrm{C}(24)-\mathrm{C}(5)-\mathrm{H}(5) & 119.2 \\ \mathrm{C}(29)-\mathrm{C}(5)-\mathrm{H}(5) & 119.2 \\ \mathrm{C}(10)-\mathrm{C}(6)-\mathrm{C}(21) & 121.77(19) \\ \mathrm{C}(10)-\mathrm{C}(6)-\mathrm{N}(1) & 120.23(18) \\ \mathrm{C}(21)-\mathrm{C}(6)-\mathrm{N}(1) & 118.0(2) \\ \mathrm{C}(18)-\mathrm{C}(7)-\mathrm{C}(25) & 118.04(19) \\ \mathrm{C}(18)-\mathrm{C}(7)-\mathrm{C}(10) & 122.52(19) \\ \mathrm{C}(25)-\mathrm{C}(7)-\mathrm{C}(10) & 119.4(2) \\ \mathrm{C}(29)-\mathrm{C}(8)-\mathrm{C}(9) & 120.26(18) \\ \mathrm{C}(29)-\mathrm{C}(8)-\mathrm{C}(10) & 117.45(18) \\ \mathrm{C}(9)-\mathrm{C}(8)-\mathrm{C}(10) & 122.17(18) \\ & \\ & \end{array}$




$\begin{array}{ll}\mathrm{C}(22)-\mathrm{C}(54)-\mathrm{Ru}(1) & 118.21(15) \\ \mathrm{C}(22)-\mathrm{C}(54)-\mathrm{H}(54) & 120.9 \\ \mathrm{Ru}(1)-\mathrm{C}(54)-\mathrm{H}(54) & 120.9 \\ \mathrm{C}(11)-\mathrm{C}(9)-\mathrm{C}(8) & 122.51(19) \\ \mathrm{C}(11)-\mathrm{C}(9)-\mathrm{C}(23) & 117.67(19) \\ \mathrm{C}(8)-\mathrm{C}(9)-\mathrm{C}(23) & 119.82(19) \\ \mathrm{C}(6)-\mathrm{C}(10)-\mathrm{C}(7) & 118.89(19) \\ \mathrm{C}(6)-\mathrm{C}(10)-\mathrm{C}(8) & 120.92(18) \\ \mathrm{C}(7)-\mathrm{C}(10)-\mathrm{C}(8) & 120.02(19) \\ \mathrm{C}(33)-\mathrm{C}(11)-\mathrm{C}(9) & 121.0(2) \\ \mathrm{C}(33)-\mathrm{C}(11)-\mathrm{H}(11) & 119.5 \\ \mathrm{C}(9)-\mathrm{C}(11)-\mathrm{H}(11) & 119.5 \\ \mathrm{C}(21)-\mathrm{C}(12)-\mathrm{C}(25) & 121.2(2) \\ \mathrm{C}(21)-\mathrm{C}(12)-\mathrm{H}(12) & 119.4 \\ \mathrm{C}(25)-\mathrm{C}(12)-\mathrm{H}(12) & 119.4 \\ \mathrm{C}(38)-\mathrm{C}(13)-\mathrm{C}(32) & 119.1(2) \\ \mathrm{C}(38)-\mathrm{C}(13)-\mathrm{C}(30) & 122.1(2) \\ \mathrm{C}(32)-\mathrm{C}(13)-\mathrm{C}(30) & 118.8(2) \\ \mathrm{O}(4)-\mathrm{C}(14)-\mathrm{C}(30) & 124.83(18) \\ \mathrm{O}(4)-\mathrm{C}(14)-\mathrm{C}(22) & 112.89(18) \\ \mathrm{C}(30)-\mathrm{C}(14)-\mathrm{C}(22) & 122.21(18) \\ \mathrm{C}(35)-\mathrm{C}(15)-\mathrm{C}(33) & 120.4(2) \\ \mathrm{C}(35)-\mathrm{C}(15)-\mathrm{H}(15) & 119.8 \\ \mathrm{C}(33)-\mathrm{C}(15)-\mathrm{H}(15) & 119.8 \\ \mathrm{C}(28)-\mathrm{C}(16)-\mathrm{C}(45) & 118.2(2) \\ \mathrm{C}(28)-\mathrm{C}(16)-\mathrm{C}(47) & 122.0(2) \\ \mathrm{C}(45)-\mathrm{C}(16)-\mathrm{C}(47) & 119.8(2) \\ \mathrm{C}(19)-\mathrm{C}(17)-\mathrm{C}(31) & 120.2(2) \\ \mathrm{C}(19)-\mathrm{C}(17)-\mathrm{H}(17) & 119.9 \\ \mathrm{C}(31)-\mathrm{C}(17)-\mathrm{H}(17) & 119.9 \\ \mathrm{C}(31)-\mathrm{C}(18)-\mathrm{C}(7) & 121.8(2) \\ \mathrm{C}(31)-\mathrm{C}(18)-\mathrm{H}(18) & 119.1 \\ \mathrm{C}(7)-\mathrm{C}(18)-\mathrm{H}(18) & 119.1 \\ \mathrm{C}(17)-\mathrm{C}(19)-\mathrm{C}(25) & 121.9(2) \\ \mathrm{C}(17)-\mathrm{C}(19)-\mathrm{H}(19) & 119.1 \\ \mathrm{C}(25)-\mathrm{C}(19)-\mathrm{H}(19) & 119.1 \\ & \\ & \end{array}$




$\begin{array}{ll}\mathrm{C}(42)-\mathrm{C}(20)-\mathrm{C}(22) & 120.1(2) \\ \mathrm{C}(42)-\mathrm{C}(20)-\mathrm{H}(20) & 119.9 \\ \mathrm{C}(22)-\mathrm{C}(20)-\mathrm{H}(20) & 119.9 \\ \mathrm{C}(12)-\mathrm{C}(21)-\mathrm{C}(6) & 119.6(2) \\ \mathrm{C}(12)-\mathrm{C}(21)-\mathrm{H}(21) & 120.2 \\ \mathrm{C}(6)-\mathrm{C}(21)-\mathrm{H}(21) & 120.2 \\ \mathrm{C}(14)-\mathrm{C}(22)-\mathrm{C}(20) & 118.3(2) \\ \mathrm{C}(14)-\mathrm{C}(22)-\mathrm{C}(54) & 119.18(18) \\ \mathrm{C}(20)-\mathrm{C}(22)-\mathrm{C}(54) & 122.4(2) \\ \mathrm{C}(35)-\mathrm{C}(23)-\mathrm{C}(24) & 121.6(2) \\ \mathrm{C}(35)-\mathrm{C}(23)-\mathrm{C}(9) & 119.7(2) \\ \mathrm{C}(24)-\mathrm{C}(23)-\mathrm{C}(9) & 118.75(19) \\ \mathrm{C}(5)-\mathrm{C}(24)-\mathrm{C}(23) & 120.6(2) \\ \mathrm{C}(5)-\mathrm{C}(24)-\mathrm{H}(24) & 119.7 \\ \mathrm{C}(23)-\mathrm{C}(24)-\mathrm{H}(24) & 119.7 \\ \mathrm{C}(12)-\mathrm{C}(25)-\mathrm{C}(19) & 122.6(2) \\ \mathrm{C}(12)-\mathrm{C}(25)-\mathrm{C}(7) & 119.1(2) \\ \mathrm{C}(19)-\mathrm{C}(25)-\mathrm{C}(7) & 118.3(2) \\ \mathrm{C}(55)-\mathrm{N}(2)-\mathrm{C}(28) & 128.0(2) \\ \mathrm{C}(55)-\mathrm{N}(2)-\mathrm{C}(51) & 113.14(18) \\ \mathrm{C}(28)-\mathrm{N}(2)-\mathrm{C}(51) & 118.86(19) \\ \mathrm{C}(41)-\mathrm{C}(27)-\mathrm{C}(45) & 118.9(2) \\ \mathrm{C}(41)-\mathrm{C}(27)-\mathrm{C}(49) & 120.6(3) \\ \mathrm{C}(45)-\mathrm{C}(27)-\mathrm{C}(49) & 120.5(3) \\ \mathrm{C}(36)-\mathrm{C}(28)-\mathrm{C}(16) & 122.1(2) \\ \mathrm{C}(36)-\mathrm{C}(28)-\mathrm{N}(2) & 119.9(2) \\ \mathrm{C}(16)-\mathrm{C}(28)-\mathrm{N}(2) & 117.9(2) \\ \mathrm{O}(1)-\mathrm{C}(29)-\mathrm{C}(8) & 122.69(18) \\ \mathrm{O}(1)-\mathrm{C}(29)-\mathrm{C}(5) & 118.08(19) \\ \mathrm{C}(8)-\mathrm{C}(29)-\mathrm{C}(5) & 118.97(19) \\ \mathrm{C}(14)-\mathrm{C}(30)-\mathrm{C}(43) & 117.3(2) \\ \mathrm{C}(14)-\mathrm{C}(30)-\mathrm{C}(13) & 123.01(19) \\ \mathrm{C}(43)-\mathrm{C}(30)-\mathrm{C}(13) & 119.5(2) \\ \mathrm{C}(18)-\mathrm{C}(31)-\mathrm{C}(17) & 119.8(2) \\ \mathrm{C}(18)-\mathrm{C}(31)-\mathrm{H}(31) & 120.1 \\ \mathrm{C}(17)-\mathrm{C}(31)-\mathrm{H}(31) & 120.1 \\ & \end{array}$




$\begin{array}{ll}\mathrm{C}(40)-\mathrm{C}(32)-\mathrm{C}(13) & 120.0(3) \\ \mathrm{C}(40)-\mathrm{C}(32)-\mathrm{H}(32) & 120.0 \\ \mathrm{C}(13)-\mathrm{C}(32)-\mathrm{H}(32) & 120.0 \\ \mathrm{C}(11)-\mathrm{C}(33)-\mathrm{C}(15) & 120.7(2) \\ \mathrm{C}(11)-\mathrm{C}(33)-\mathrm{H}(33) & 119.7 \\ \mathrm{C}(15)-\mathrm{C}(33)-\mathrm{H}(33) & 119.7 \\ \mathrm{O}(4)-\mathrm{C}(34)-\mathrm{C}(37) & 109.76(18) \\ \mathrm{O}(4)-\mathrm{C}(34)-\mathrm{C}(50) & 105.51(18) \\ \mathrm{C}(37)-\mathrm{C}(34)-\mathrm{C}(50) & 115.5(2) \\ \mathrm{O}(4)-\mathrm{C}(34)-\mathrm{H}(34) & 108.6 \\ \mathrm{C}(37)-\mathrm{C}(34)-\mathrm{H}(34) & 108.6 \\ \mathrm{C}(50)-\mathrm{C}(34)-\mathrm{H}(34) & 108.6 \\ \mathrm{C}(15)-\mathrm{C}(35)-\mathrm{C}(23) & 120.6(2) \\ \mathrm{C}(15)-\mathrm{C}(35)-\mathrm{H}(35) & 119.7 \\ \mathrm{C}(23)-\mathrm{C}(35)-\mathrm{H}(35) & 119.7 \\ \mathrm{C}(28)-\mathrm{C}(36)-\mathrm{C}(41) & 117.2(2) \\ \mathrm{C}(28)-\mathrm{C}(36)-\mathrm{C}(48) & 122.9(2) \\ \mathrm{C}(41)-\mathrm{C}(36)-\mathrm{C}(48) & 119.9(2) \\ \mathrm{C}(34)-\mathrm{C}(37)-\mathrm{H}(37 \mathrm{~A}) & 109.5 \\ \mathrm{C}(34)-\mathrm{C}(37)-\mathrm{H}(37 \mathrm{~B}) & 109.5 \\ \mathrm{H}(37 \mathrm{~A})-\mathrm{C}(37)-\mathrm{H}(37 \mathrm{~B}) & 109.5 \\ \mathrm{C}(34)-\mathrm{C}(37)-\mathrm{H}(37 \mathrm{C}) & 109.5 \\ \mathrm{H}(37 \mathrm{~A})-\mathrm{C}(37)-\mathrm{H}(37 \mathrm{C}) & 109.5 \\ \mathrm{H}(37 \mathrm{~B})-\mathrm{C}(37)-\mathrm{H}(37 \mathrm{C}) & 109.5 \\ \mathrm{C}(39)-\mathrm{C}(38)-\mathrm{C}(13) & 120.2(3) \\ \mathrm{C}(39)-\mathrm{C}(38)-\mathrm{H}(38) & 119.9 \\ \mathrm{C}(13)-\mathrm{C}(38)-\mathrm{H}(38) & 119.9 \\ \mathrm{C}(38)-\mathrm{C}(39)-\mathrm{C}(44) & 120.3(3) \\ \mathrm{C}(38)-\mathrm{C}(39)-\mathrm{H}(39) & 119.9 \\ \mathrm{C}(44)-\mathrm{C}(39)-\mathrm{H}(39) & 119.9 \\ \mathrm{C}(44)-\mathrm{C}(40)-\mathrm{C}(32) & 120.2(3) \\ \mathrm{C}(44)-\mathrm{C}(40)-\mathrm{H}(40) & 119.9 \\ \mathrm{C}(32)-\mathrm{C}(40)-\mathrm{H}(40) & 119.9 \\ \mathrm{C}(27)-\mathrm{C}(41)-\mathrm{C}(36) & 122.1(3) \\ \mathrm{C}(27)-\mathrm{C}(41)-\mathrm{H}(41) & 118.9 \\ \mathrm{C}(36)-\mathrm{C}(41)-\mathrm{H}(41) & 118.9 \\ & \\ & \end{array}$




\begin{tabular}{|c|c|}
\hline$C(20)-C(42)-C(43)$ & $120.2(2)$ \\
\hline $\mathrm{C}(20)-\mathrm{C}(42)-\mathrm{H}(42)$ & 119.9 \\
\hline $\mathrm{C}(43)-\mathrm{C}(42)-\mathrm{H}(42)$ & 119.9 \\
\hline $\mathrm{C}(42)-\mathrm{C}(43)-\mathrm{C}(30)$ & $121.4(2)$ \\
\hline $\mathrm{C}(42)-\mathrm{C}(43)-\mathrm{H}(43)$ & 119.3 \\
\hline $\mathrm{C}(30)-\mathrm{C}(43)-\mathrm{H}(43)$ & 119.3 \\
\hline $\mathrm{C}(40)-\mathrm{C}(44)-\mathrm{C}(39)$ & $120.1(3)$ \\
\hline $\mathrm{C}(40)-\mathrm{C}(44)-\mathrm{H}(44)$ & 120.0 \\
\hline $\mathrm{C}(39)-\mathrm{C}(44)-\mathrm{H}(44)$ & 120.0 \\
\hline $\mathrm{C}(27)-\mathrm{C}(45)-\mathrm{C}(16)$ & $121.4(3)$ \\
\hline $\mathrm{C}(27)-\mathrm{C}(45)-\mathrm{H}(45)$ & 119.3 \\
\hline $\mathrm{C}(16)-\mathrm{C}(45)-\mathrm{H}(45)$ & 119.3 \\
\hline $\mathrm{C}(29)-\mathrm{O}(1)-\mathrm{Ru}(1)$ & $128.02(13)$ \\
\hline $\mathrm{C}(55)-\mathrm{N}(1)-\mathrm{C}(6)$ & $123.47(19)$ \\
\hline$C(55)-N(1)-C(52)$ & $113.76(18)$ \\
\hline $\mathrm{C}(6)-\mathrm{N}(1)-\mathrm{C}(52)$ & $121.01(18)$ \\
\hline $\mathrm{N}(2)-\mathrm{C}(55)-\mathrm{N}(1)$ & $106.32(19)$ \\
\hline $\mathrm{N}(2)-\mathrm{C}(55)-\mathrm{Ru}(1)$ & $136.44(16)$ \\
\hline $\mathrm{N}(1)-\mathrm{C}(55)-\mathrm{Ru}(1)$ & $117.20(15)$ \\
\hline $\mathrm{C}(34)-\mathrm{C}(50)-\mathrm{H}(50 \mathrm{~A})$ & 109.5 \\
\hline $\mathrm{C}(34)-\mathrm{C}(50)-\mathrm{H}(50 \mathrm{~B})$ & 109.5 \\
\hline $\mathrm{H}(50 \mathrm{~A})-\mathrm{C}(50)-\mathrm{H}(50 \mathrm{~B})$ & 109.5 \\
\hline $\mathrm{C}(34)-\mathrm{C}(50)-\mathrm{H}(50 \mathrm{C})$ & 109.5 \\
\hline $\mathrm{H}(50 \mathrm{~A})-\mathrm{C}(50)-\mathrm{H}(50 \mathrm{C})$ & 109.5 \\
\hline $\mathrm{H}(50 \mathrm{~B})-\mathrm{C}(50)-\mathrm{H}(50 \mathrm{C})$ & 109.5 \\
\hline $\mathrm{C}(27)-\mathrm{C}(49)-\mathrm{H}(49 \mathrm{~A})$ & 109.5 \\
\hline $\mathrm{C}(27)-\mathrm{C}(49)-\mathrm{H}(49 \mathrm{~B})$ & 109.5 \\
\hline $\mathrm{H}(49 \mathrm{~A})-\mathrm{C}(49)-\mathrm{H}(49 \mathrm{~B})$ & 109.5 \\
\hline $\mathrm{C}(27)-\mathrm{C}(49)-\mathrm{H}(49 \mathrm{C})$ & 109.5 \\
\hline $\mathrm{H}(49 \mathrm{~A})-\mathrm{C}(49)-\mathrm{H}(49 \mathrm{C})$ & 109.5 \\
\hline H(49B)-C(49)-H(49C) & 109.5 \\
\hline $\mathrm{N}(1)-\mathrm{C}(52)-\mathrm{C}(51)$ & $101.74(19)$ \\
\hline $\mathrm{N}(1)-\mathrm{C}(52)-\mathrm{H}(52 \mathrm{~A})$ & 111.4 \\
\hline $\mathrm{C}(51)-\mathrm{C}(52)-\mathrm{H}(52 \mathrm{~A})$ & 111.4 \\
\hline $\mathrm{N}(1)-\mathrm{C}(52)-\mathrm{H}(52 \mathrm{~B})$ & 111.4 \\
\hline $\mathrm{C}(51)-\mathrm{C}(52)-\mathrm{H}(52 \mathrm{~B})$ & 111.4 \\
\hline
\end{tabular}




$\begin{array}{ll}\mathrm{H}(52 \mathrm{~A})-\mathrm{C}(52)-\mathrm{H}(52 \mathrm{~B}) & 109.3 \\ \mathrm{~N}(2)-\mathrm{C}(51)-\mathrm{C}(52) & 102.58(19) \\ \mathrm{N}(2)-\mathrm{C}(51)-\mathrm{H}(51 \mathrm{~A}) & 111.3 \\ \mathrm{C}(52)-\mathrm{C}(51)-\mathrm{H}(51 \mathrm{~A}) & 111.3 \\ \mathrm{~N}(2)-\mathrm{C}(51)-\mathrm{H}(51 \mathrm{~B}) & 111.3 \\ \mathrm{C}(52)-\mathrm{C}(51)-\mathrm{H}(51 \mathrm{~B}) & 111.3 \\ \mathrm{H}(51 \mathrm{~A})-\mathrm{C}(51)-\mathrm{H}(51 \mathrm{~B}) & 109.2 \\ \mathrm{C}(36)-\mathrm{C}(48)-\mathrm{H}(48 \mathrm{~A}) & 109.5 \\ \mathrm{C}(36)-\mathrm{C}(48)-\mathrm{H}(48 \mathrm{~B}) & 109.5 \\ \mathrm{H}(48 \mathrm{~A})-\mathrm{C}(48)-\mathrm{H}(48 \mathrm{~B}) & 109.5 \\ \mathrm{C}(36)-\mathrm{C}(48)-\mathrm{H}(48 \mathrm{C}) & 109.5 \\ \mathrm{H}(48 \mathrm{~A})-\mathrm{C}(48)-\mathrm{H}(48 \mathrm{C}) & 109.5 \\ \mathrm{H}(48 \mathrm{~B})-\mathrm{C}(48)-\mathrm{H}(48 \mathrm{C}) & 109.5 \\ \mathrm{C}(16)-\mathrm{C}(47)-\mathrm{H}(47 \mathrm{~A}) & 109.5 \\ \mathrm{C}(16)-\mathrm{C}(47)-\mathrm{H}(47 \mathrm{~B}) & 109.5 \\ \mathrm{H}(47 \mathrm{~A})-\mathrm{C}(47)-\mathrm{H}(47 \mathrm{~B}) & 109.5 \\ \mathrm{C}(16)-\mathrm{C}(47)-\mathrm{H}(47 \mathrm{C}) & 109.5 \\ \mathrm{H}(47 \mathrm{~A})-\mathrm{C}(47)-\mathrm{H}(47 \mathrm{C}) & 109.5 \\ \mathrm{H}(47 \mathrm{~B})-\mathrm{C}(47)-\mathrm{H}(47 \mathrm{C}) & 109.5\end{array}$

Table 4. Anisotropic displacement parameters $\left(\approx^{2} \times 10^{3}\right)$ for DG01t. The anisotropic displacement factor exponent takes the form: $-2 p^{2}\left[h^{2} a^{* 2} U^{11}+\ldots+2\right.$ $\mathbf{h ~ k} \mathbf{a}^{*} \mathbf{b}^{*} \mathbf{U}^{12}$ ]

\begin{tabular}{lcccccc}
\hline & $\mathrm{U}^{11}$ & $\mathrm{U}^{22}$ & $\mathrm{U}^{33}$ & $\mathrm{U}^{23}$ & $\mathrm{U}^{13}$ & $\mathrm{U}^{12}$ \\
\hline $\mathrm{Ru}(1)$ & $24(1)$ & $25(1)$ & $19(1)$ & $0(1)$ & $0(1)$ & $-2(1)$ \\
$\mathrm{I}(2)$ & $33(1)$ & $39(1)$ & $35(1)$ & $2(1)$ & $-4(1)$ & $7(1)$ \\
$\mathrm{O}(4)$ & $32(1)$ & $29(1)$ & $17(1)$ & $0(1)$ & $0(1)$ & $-4(1)$ \\
$\mathrm{C}(5)$ & $33(1)$ & $33(1)$ & $25(1)$ & $-6(1)$ & $4(1)$ & $4(1)$ \\
$\mathrm{C}(6)$ & $25(1)$ & $26(1)$ & $22(1)$ & $-3(1)$ & $1(1)$ & $-3(1)$ \\
$\mathrm{C}(7)$ & $22(1)$ & $26(1)$ & $23(1)$ & $-6(1)$ & $1(1)$ & $-2(1)$ \\
$\mathrm{C}(8)$ & $23(1)$ & $27(1)$ & $21(1)$ & $1(1)$ & $2(1)$ & $1(1)$ \\
$\mathrm{C}(54)$ & $28(1)$ & $34(1)$ & $24(1)$ & $1(1)$ & $3(1)$ & $-5(1)$ \\
$\mathrm{C}(9)$ & $23(1)$ & $25(1)$ & $23(1)$ & $2(1)$ & $-1(1)$ & $1(1)$
\end{tabular}




\begin{tabular}{|c|c|c|c|c|c|c|}
\hline$C(10)$ & $23(1)$ & $26(1)$ & $22(1)$ & $-6(1)$ & $-1(1)$ & $0(1)$ \\
\hline $\mathrm{C}(11)$ & $30(1)$ & $28(1)$ & $25(1)$ & $0(1)$ & $-2(1)$ & $2(1)$ \\
\hline$C(12)$ & $25(1)$ & $39(1)$ & $33(1)$ & $-4(1)$ & $3(1)$ & $7(1)$ \\
\hline$C(13)$ & $29(1)$ & $37(1)$ & $22(1)$ & $-4(1)$ & $-5(1)$ & $-6(1)$ \\
\hline$C(14)$ & $26(1)$ & $30(1)$ & $18(1)$ & $-3(1)$ & $-1(1)$ & $1(1)$ \\
\hline$C(15)$ & $28(1)$ & $49(2)$ & $39(1)$ & $4(1)$ & $-13(1)$ & $-2(1)$ \\
\hline$C(16)$ & $35(1)$ & $41(1)$ & $38(1)$ & $6(1)$ & $4(1)$ & $-7(1)$ \\
\hline$C(17)$ & $40(1)$ & $50(2)$ & $30(1)$ & $3(1)$ & 11(1) & $-8(1)$ \\
\hline $\mathrm{C}(18)$ & $28(1)$ & $33(1)$ & $28(1)$ & $1(1)$ & $4(1)$ & $2(1)$ \\
\hline$C(19)$ & $31(1)$ & $43(1)$ & $32(1)$ & $-3(1)$ & $9(1)$ & $-2(1)$ \\
\hline$C(20)$ & $32(1)$ & $46(2)$ & $20(1)$ & $-2(1)$ & $4(1)$ & $-2(1)$ \\
\hline $\mathrm{C}(21)$ & $30(1)$ & $29(1)$ & $31(1)$ & $-3(1)$ & $-2(1)$ & $6(1)$ \\
\hline$C(22)$ & $28(1)$ & $32(1)$ & $21(1)$ & $-2(1)$ & $-1(1)$ & $0(1)$ \\
\hline$C(23)$ & $24(1)$ & $36(1)$ & $27(1)$ & $1(1)$ & $1(1)$ & $2(1)$ \\
\hline$C(24)$ & $28(1)$ & $40(1)$ & $30(1)$ & $-5(1)$ & $3(1)$ & $9(1)$ \\
\hline$C(25)$ & $23(1)$ & $34(1)$ & $28(1)$ & $-6(1)$ & $1(1)$ & $-1(1)$ \\
\hline $\mathrm{N}(2)$ & $42(1)$ & $34(1)$ & $29(1)$ & $-1(1)$ & $5(1)$ & $-13(1)$ \\
\hline $\mathrm{C}(27)$ & $41(1)$ & $61(2)$ & $33(1)$ & $-6(1)$ & $6(1)$ & $-13(1)$ \\
\hline $\mathrm{C}(28)$ & $36(1)$ & $34(1)$ & $29(1)$ & $2(1)$ & $5(1)$ & $-10(1)$ \\
\hline$C(29)$ & $25(1)$ & $26(1)$ & $24(1)$ & $1(1)$ & $1(1)$ & $0(1)$ \\
\hline$C(30)$ & $27(1)$ & $30(1)$ & $26(1)$ & $-1(1)$ & $-3(1)$ & $-1(1)$ \\
\hline $\mathrm{C}(31)$ & $39(1)$ & $41(1)$ & $31(1)$ & $7(1)$ & $0(1)$ & $1(1)$ \\
\hline$C(32)$ & $33(1)$ & $42(1)$ & $31(1)$ & $0(1)$ & $-2(1)$ & $0(1)$ \\
\hline $\mathrm{C}(33)$ & $38(1)$ & $34(1)$ & $32(1)$ & $-1(1)$ & $-7(1)$ & $-6(1)$ \\
\hline$C(34)$ & $34(1)$ & $32(1)$ & $22(1)$ & $2(1)$ & $0(1)$ & $-9(1)$ \\
\hline$C(35)$ & $22(1)$ & $49(2)$ & $37(1)$ & $3(1)$ & $-2(1)$ & $5(1)$ \\
\hline$C(36)$ & $39(1)$ & $38(1)$ & $36(1)$ & $3(1)$ & $7(1)$ & $-4(1)$ \\
\hline$C(37)$ & $58(2)$ & $32(1)$ & $29(1)$ & $2(1)$ & $-6(1)$ & $1(1)$ \\
\hline $\mathrm{C}(38)$ & $43(1)$ & $38(1)$ & $41(1)$ & $-7(1)$ & $-2(1)$ & $-8(1)$ \\
\hline$C(39)$ & $56(2)$ & $47(2)$ & $53(2)$ & $2(1)$ & $2(1)$ & $-19(1)$ \\
\hline $\mathrm{C}(40)$ & $32(1)$ & $65(2)$ & $40(1)$ & $-3(1)$ & $4(1)$ & $1(1)$ \\
\hline $\mathrm{C}(41)$ & $41(1)$ & $61(2)$ & $29(1)$ & $3(1)$ & 1(1) & $-8(1)$ \\
\hline$C(42)$ & $40(1)$ & $49(2)$ & $23(1)$ & $-6(1)$ & $1(1)$ & $2(1)$ \\
\hline $\mathrm{C}(43)$ & $32(1)$ & $43(2)$ & $26(1)$ & $-9(1)$ & $-5(1)$ & $-1(1)$ \\
\hline $\mathrm{C}(44)$ & $38(1)$ & $79(2)$ & $44(2)$ & $7(1)$ & $7(1)$ & $-17(1)$ \\
\hline$C(45)$ & $36(1)$ & $47(2)$ & $46(1)$ & $-3(1)$ & $9(1)$ & $-6(1)$ \\
\hline
\end{tabular}




$\begin{array}{lllllll}\mathrm{O}(1) & 28(1) & 27(1) & 24(1) & 0(1) & -4(1) & -3(1) \\ \mathrm{N}(1) & 29(1) & 25(1) & 24(1) & 0(1) & -1(1) & -4(1) \\ \mathrm{C}(55) & 25(1) & 28(1) & 24(1) & 2(1) & 0(1) & 2(1) \\ \mathrm{C}(50) & 44(1) & 42(1) & 23(1) & 1(1) & 6(1) & -7(1) \\ \mathrm{C}(49) & 53(2) & 108(3) & 36(1) & -18(2) & 8(1) & -13(2) \\ \mathrm{C}(52) & 58(2) & 44(2) & 36(1) & -11(1) & 8(1) & -25(1) \\ \mathrm{C}(51) & 50(2) & 37(1) & 38(1) & -4(1) & 4(1) & -18(1) \\ \mathrm{C}(48) & 47(2) & 62(2) & 47(2) & 10(1) & 7(1) & 7(1) \\ \mathrm{C}(47) & 52(2) & 71(2) & 48(2) & 19(2) & 6(1) & 9(2)\end{array}$

Table 5. Hydrogen coordinates ( $x 1^{4}$ ) and isotropic displacement parameters $\left(\approx^{2} \times 1^{3}\right)$ for DG01t.

\begin{tabular}{|c|c|c|c|c|}
\hline & $\mathrm{x}$ & $\mathrm{y}$ & $\mathrm{z}$ & $\mathrm{U}(\mathrm{eq})$ \\
\hline $\mathrm{H}(5)$ & -84 & 8716 & 7209 & 36 \\
\hline $\mathrm{H}(54)$ & 1833 & 6319 & 8857 & 35 \\
\hline $\mathrm{H}(11)$ & 214 & 4971 & 4732 & 33 \\
\hline $\mathrm{H}(12)$ & 4789 & 4584 & 5201 & 39 \\
\hline $\mathrm{H}(15)$ & -3189 & 5800 & 4527 & 47 \\
\hline $\mathrm{H}(17)$ & 3980 & 7913 & 3401 & 48 \\
\hline $\mathrm{H}(18)$ & 1169 & 7776 & 4676 & 36 \\
\hline $\mathrm{H}(19)$ & 4843 & 6248 & 4129 & 42 \\
\hline $\mathrm{H}(20)$ & 2331 & 7700 & 10055 & 39 \\
\hline $\mathrm{H}(21)$ & 3833 & 3634 & 6197 & 36 \\
\hline $\mathrm{H}(24)$ & -1818 & 8481 & 6541 & 39 \\
\hline $\mathrm{H}(31)$ & 2122 & 8687 & 3668 & 45 \\
\hline $\mathrm{H}(32)$ & 6477 & 8018 & 8519 & 43 \\
\hline $\mathrm{H}(33)$ & -1566 & 4657 & 4119 & 42 \\
\hline $\mathrm{H}(34)$ & 5063 & 9567 & 7445 & 35 \\
\hline $\mathrm{H}(35)$ & -3031 & 7292 & 5543 & 44 \\
\hline $\mathrm{H}(37 \mathrm{~A})$ & 2643 & 9918 & 7157 & 60 \\
\hline $\mathrm{H}(37 \mathrm{~B})$ & 3601 & 10968 & 6901 & 60 \\
\hline $\mathrm{H}(37 \mathrm{C})$ & 3404 & 10725 & 7789 & 60 \\
\hline $\mathrm{H}(38)$ & 5477 & 11911 & 8788 & 49 \\
\hline
\end{tabular}




\begin{tabular}{lrrrl}
$\mathrm{H}(39)$ & 7094 & 12632 & 8165 & 62 \\
$\mathrm{H}(40)$ & 8112 & 8765 & 7921 & 55 \\
$\mathrm{H}(41)$ & 1443 & 3563 & 10379 & 52 \\
$\mathrm{H}(42)$ & 3454 & 9363 & 10669 & 45 \\
$\mathrm{H}(43)$ & 4986 & 10337 & 10057 & 40 \\
$\mathrm{H}(44)$ & 8410 & 11063 & 7734 & 64 \\
$\mathrm{H}(45)$ & -1302 & 5629 & 9534 & 51 \\
$\mathrm{H}(50 \mathrm{~A})$ & 4837 & 7573 & 6650 & 54 \\
$\mathrm{H}(50 \mathrm{~B})$ & 4760 & 8963 & 6183 & 54 \\
$\mathrm{H}(50 \mathrm{C})$ & 3609 & 8151 & 6356 & 54 \\
$\mathrm{H}(49 \mathrm{~A})$ & 49 & 5637 & 11102 & 98 \\
$\mathrm{H}(49 \mathrm{~B})$ & -218 & 4058 & 11185 & 98 \\
$\mathrm{H}(49 \mathrm{C})$ & -1201 & 5062 & 10857 & 98 \\
$\mathrm{H}(52 \mathrm{~A})$ & 1338 & 2387 & 6349 & 55 \\
$\mathrm{H}(52 \mathrm{~B})$ & 321 & 3498 & 6272 & 55 \\
$\mathrm{H}(51 \mathrm{~A})$ & -195 & 2798 & 7433 & 49 \\
$\mathrm{H}(51 \mathrm{~B})$ & 1010 & 2012 & 7590 & 49 \\
$\mathrm{H}(48 \mathrm{~A})$ & 2494 & 2519 & 8605 & 78 \\
$\mathrm{H}(48 \mathrm{~B})$ & 2581 & 2210 & 9503 & 78 \\
$\mathrm{H}(48 \mathrm{C})$ & 3160 & 3546 & 9176 & 78 \\
$\mathrm{H}(47 \mathrm{~A})$ & -498 & 5961 & 7691 & 86 \\
$\mathrm{H}(47 \mathrm{~B})$ & -1570 & 6114 & 8228 & 86 \\
$\mathrm{H}(47 \mathrm{C})$ & -1388 & 4738 & 7767 & 86 \\
& & & & \\
\hline
\end{tabular}

Table 6. Torsion angles [®] for DG01t.

\begin{tabular}{lc}
\hline $\mathrm{C}(54)-\mathrm{Ru}(1)-\mathrm{O}(4)-\mathrm{C}(14)$ & $6.83(14)$ \\
$\mathrm{C}(55)-\mathrm{Ru}(1)-\mathrm{O}(4)-\mathrm{C}(14)$ & $-95.2(12)$ \\
$\mathrm{O}(1)-\mathrm{Ru}(1)-\mathrm{O}(4)-\mathrm{C}(14)$ & $114.15(13)$ \\
$\mathrm{I}(2)-\mathrm{Ru}(1)-\mathrm{O}(4)-\mathrm{C}(14)$ & $-95.29(12)$ \\
$\mathrm{C}(54)-\mathrm{Ru}(1)-\mathrm{O}(4)-\mathrm{C}(34)$ & $-143.79(17)$ \\
$\mathrm{C}(55)-\mathrm{Ru}(1)-\mathrm{O}(4)-\mathrm{C}(34)$ & $114.1(12)$ \\
$\mathrm{O}(1)-\mathrm{Ru}(1)-\mathrm{O}(4)-\mathrm{C}(34)$ & $-36.47(15)$ \\
$\mathrm{I}(2)-\mathrm{Ru}(1)-\mathrm{O}(4)-\mathrm{C}(34)$ & $114.10(15)$ \\
$\mathrm{C}(55)-\mathrm{Ru}(1)-\mathrm{C}(54)-\mathrm{C}(22)$ & $173.30(18)$
\end{tabular}




$\begin{array}{lc}\mathrm{O}(1)-\mathrm{Ru}(1)-\mathrm{C}(54)-\mathrm{C}(22) & -88.06(19) \\ \mathrm{O}(4)-\mathrm{Ru}(1)-\mathrm{C}(54)-\mathrm{C}(22) & -3.56(17) \\ \mathrm{I}(2)-\mathrm{Ru}(1)-\mathrm{C}(54)-\mathrm{C}(22) & 78.45(18) \\ \mathrm{C}(29)-\mathrm{C}(8)-\mathrm{C}(9)-\mathrm{C}(11) & -178.5(2) \\ \mathrm{C}(10)-\mathrm{C}(8)-\mathrm{C}(9)-\mathrm{C}(11) & 5.7(3) \\ \mathrm{C}(29)-\mathrm{C}(8)-\mathrm{C}(9)-\mathrm{C}(23) & 1.1(3) \\ \mathrm{C}(10)-\mathrm{C}(8)-\mathrm{C}(9)-\mathrm{C}(23) & -174.8(2) \\ \mathrm{C}(21)-\mathrm{C}(6)-\mathrm{C}(10)-\mathrm{C}(7) & 1.8(3) \\ \mathrm{N}(1)-\mathrm{C}(6)-\mathrm{C}(10)-\mathrm{C}(7) & -176.36(19) \\ \mathrm{C}(21)-\mathrm{C}(6)-\mathrm{C}(10)-\mathrm{C}(8) & -173.42(19) \\ \mathrm{N}(1)-\mathrm{C}(6)-\mathrm{C}(10)-\mathrm{C}(8) & 8.4(3) \\ \mathrm{C}(18)-\mathrm{C}(7)-\mathrm{C}(10)-\mathrm{C}(6) & 177.7(2) \\ \mathrm{C}(25)-\mathrm{C}(7)-\mathrm{C}(10)-\mathrm{C}(6) & -3.4(3) \\ \mathrm{C}(18)-\mathrm{C}(7)-\mathrm{C}(10)-\mathrm{C}(8) & -7.0(3) \\ \mathrm{C}(25)-\mathrm{C}(7)-\mathrm{C}(10)-\mathrm{C}(8) & 171.9(2) \\ \mathrm{C}(29)-\mathrm{C}(8)-\mathrm{C}(10)-\mathrm{C}(6) & 73.6(3) \\ \mathrm{C}(9)-\mathrm{C}(8)-\mathrm{C}(10)-\mathrm{C}(6) & -110.4(2) \\ \mathrm{C}(29)-\mathrm{C}(8)-\mathrm{C}(10)-\mathrm{C}(7) & -101.6(2) \\ \mathrm{C}(9)-\mathrm{C}(8)-\mathrm{C}(10)-\mathrm{C}(7) & 74.3(3) \\ \mathrm{C}(8)-\mathrm{C}(9)-\mathrm{C}(11)-\mathrm{C}(33) & 178.2(2) \\ \mathrm{C}(23)-\mathrm{C}(9)-\mathrm{C}(11)-\mathrm{C}(33) & -1.3(3) \\ \mathrm{C}(34)-\mathrm{O}(4)-\mathrm{C}(14)-\mathrm{C}(30) & -39.9(3) \\ \mathrm{Ru}(1)-\mathrm{O}(4)-\mathrm{C}(14)-\mathrm{C}(30) & 168.36(19) \\ \mathrm{C}(34)-\mathrm{O}(4)-\mathrm{C}(14)-\mathrm{C}(22) & 143.28(19) \\ \mathrm{Ru}(1)-\mathrm{O}(4)-\mathrm{C}(14)-\mathrm{C}(22) & -8.5(2) \\ \mathrm{C}(25)-\mathrm{C}(7)-\mathrm{C}(18)-\mathrm{C}(31) & -1.6(4) \\ \mathrm{C}(10)-\mathrm{C}(7)-\mathrm{C}(18)-\mathrm{C}(31) & 177.3(2) \\ \mathrm{C}(31)-\mathrm{C}(17)-\mathrm{C}(19)-\mathrm{C}(25) & 0.4(4) \\ \mathrm{C}(25)-\mathrm{C}(12)-\mathrm{C}(21)-\mathrm{C}(6) & -2.3(4) \\ \mathrm{C}(10)-\mathrm{C}(6)-\mathrm{C}(21)-\mathrm{C}(12) & 1.0(3) \\ \mathrm{N}(1)-\mathrm{C}(6)-\mathrm{C}(21)-\mathrm{C}(12) & 179.3(2) \\ \mathrm{O}(4)-\mathrm{C}(14)-\mathrm{C}(22)-\mathrm{C}(20) & -177.8(2) \\ \mathrm{C}(30)-\mathrm{C}(14)-\mathrm{C}(22)-\mathrm{C}(20) & 5.3(3) \\ \mathrm{O}(4)-\mathrm{C}(14)-\mathrm{C}(22)-\mathrm{C}(54) & 6.5(3) \\ \mathrm{C}(30)-\mathrm{C}(14)-\mathrm{C}(22)-\mathrm{C}(54) & -170.4(2) \\ \mathrm{C}(42)-\mathrm{C}(20)-\mathrm{C}(22)-\mathrm{C}(14) & \\ & \\ & \\ & \end{array}$




$\begin{array}{lc}\mathrm{C}(42)-\mathrm{C}(20)-\mathrm{C}(22)-\mathrm{C}(54) & 176.3(2) \\ \mathrm{Ru}(1)-\mathrm{C}(54)-\mathrm{C}(22)-\mathrm{C}(14) & -0.2(3) \\ \mathrm{Ru}(1)-\mathrm{C}(54)-\mathrm{C}(22)-\mathrm{C}(20) & -175.66(18) \\ \mathrm{C}(11)-\mathrm{C}(9)-\mathrm{C}(23)-\mathrm{C}(35) & 1.4(3) \\ \mathrm{C}(8)-\mathrm{C}(9)-\mathrm{C}(23)-\mathrm{C}(35) & -178.2(2) \\ \mathrm{C}(11)-\mathrm{C}(9)-\mathrm{C}(23)-\mathrm{C}(24) & -178.4(2) \\ \mathrm{C}(8)-\mathrm{C}(9)-\mathrm{C}(23)-\mathrm{C}(24) & 2.1(3) \\ \mathrm{C}(29)-\mathrm{C}(5)-\mathrm{C}(24)-\mathrm{C}(23) & 1.5(4) \\ \mathrm{C}(35)-\mathrm{C}(23)-\mathrm{C}(24)-\mathrm{C}(5) & 176.9(2) \\ \mathrm{C}(9)-\mathrm{C}(23)-\mathrm{C}(24)-\mathrm{C}(5) & -3.4(4) \\ \mathrm{C}(21)-\mathrm{C}(12)-\mathrm{C}(25)-\mathrm{C}(19) & 179.4(2) \\ \mathrm{C}(21)-\mathrm{C}(12)-\mathrm{C}(25)-\mathrm{C}(7) & 0.7(4) \\ \mathrm{C}(17)-\mathrm{C}(19)-\mathrm{C}(25)-\mathrm{C}(12) & 179.4(2) \\ \mathrm{C}(17)-\mathrm{C}(19)-\mathrm{C}(25)-\mathrm{C}(7) & -1.9(4) \\ \mathrm{C}(18)-\mathrm{C}(7)-\mathrm{C}(25)-\mathrm{C}(12) & -178.8(2) \\ \mathrm{C}(10)-\mathrm{C}(7)-\mathrm{C}(25)-\mathrm{C}(12) & 2.2(3) \\ \mathrm{C}(18)-\mathrm{C}(7)-\mathrm{C}(25)-\mathrm{C}(19) & 2.4(3) \\ \mathrm{C}(10)-\mathrm{C}(7)-\mathrm{C}(25)-\mathrm{C}(19) & -176.5(2) \\ \mathrm{C}(45)-\mathrm{C}(16)-\mathrm{C}(28)-\mathrm{C}(36) & 3.6(4) \\ \mathrm{C}(47)-\mathrm{C}(16)-\mathrm{C}(28)-\mathrm{C}(36) & -177.9(3) \\ \mathrm{C}(45)-\mathrm{C}(16)-\mathrm{C}(28)-\mathrm{N}(2) & -179.8(2) \\ \mathrm{C}(47)-\mathrm{C}(16)-\mathrm{C}(28)-\mathrm{N}(2) & -1.3(4) \\ \mathrm{C}(55)-\mathrm{N}(2)-\mathrm{C}(28)-\mathrm{C}(36) & -89.6(3) \\ \mathrm{C}(51)-\mathrm{N}(2)-\mathrm{C}(28)-\mathrm{C}(36) & 91.5(3) \\ \mathrm{C}(55)-\mathrm{N}(2)-\mathrm{C}(28)-\mathrm{C}(16) & 93.7(3) \\ \mathrm{C}(51)-\mathrm{N}(2)-\mathrm{C}(28)-\mathrm{C}(16) & -85.1(3) \\ \mathrm{C}(9)-\mathrm{C}(8)-\mathrm{C}(29)-\mathrm{O}(1) & -176.84(19) \\ \mathrm{C}(10)-\mathrm{C}(8)-\mathrm{C}(29)-\mathrm{O}(1) & -0.8(3) \\ \mathrm{C}(9)-\mathrm{C}(8)-\mathrm{C}(29)-\mathrm{C}(5) & -2.9(3) \\ \mathrm{C}(10)-\mathrm{C}(8)-\mathrm{C}(29)-\mathrm{C}(5) & 17.97 .6(3) \\ \mathrm{C}(24)-\mathrm{C}(5)-\mathrm{C}(29)-\mathrm{O}(1) & 17.1(2) \\ \mathrm{C}(24)-\mathrm{C}(5)-\mathrm{C}(29)-\mathrm{C}(8) & 1.6(3) \\ \mathrm{O}(4)-\mathrm{C}(14)-\mathrm{C}(30)-\mathrm{C}(43) & \\ \mathrm{C}(22)-\mathrm{C}(14)-\mathrm{C}(30)-\mathrm{C}(43) & \\ \mathrm{O}(4)-\mathrm{C}(14)-\mathrm{C}(30)-\mathrm{C}(13) & \\ \mathrm{C}(22)-\mathrm{C}(14)-\mathrm{C}(30)-\mathrm{C}(13) & \\ & \\ & \\ & \end{array}$




$\begin{array}{lc}\mathrm{C}(38)-\mathrm{C}(13)-\mathrm{C}(30)-\mathrm{C}(14) & 121.9(3) \\ \mathrm{C}(32)-\mathrm{C}(13)-\mathrm{C}(30)-\mathrm{C}(14) & -57.2(3) \\ \mathrm{C}(38)-\mathrm{C}(13)-\mathrm{C}(30)-\mathrm{C}(43) & -63.1(3) \\ \mathrm{C}(32)-\mathrm{C}(13)-\mathrm{C}(30)-\mathrm{C}(43) & 117.7(2) \\ \mathrm{C}(7)-\mathrm{C}(18)-\mathrm{C}(31)-\mathrm{C}(17) & 0.1(4) \\ \mathrm{C}(19)-\mathrm{C}(17)-\mathrm{C}(31)-\mathrm{C}(18) & 0.5(4) \\ \mathrm{C}(38)-\mathrm{C}(13)-\mathrm{C}(32)-\mathrm{C}(40) & -2.5(3) \\ \mathrm{C}(30)-\mathrm{C}(13)-\mathrm{C}(32)-\mathrm{C}(40) & 176.7(2) \\ \mathrm{C}(9)-\mathrm{C}(11)-\mathrm{C}(33)-\mathrm{C}(15) & 0.3(4) \\ \mathrm{C}(35)-\mathrm{C}(15)-\mathrm{C}(33)-\mathrm{C}(11) & 0.6(4) \\ \mathrm{C}(14)-\mathrm{O}(4)-\mathrm{C}(34)-\mathrm{C}(37) & -60.7(2) \\ \mathrm{Ru}(1)-\mathrm{O}(4)-\mathrm{C}(34)-\mathrm{C}(37) & 86.80(19) \\ \mathrm{C}(14)-\mathrm{O}(4)-\mathrm{C}(34)-\mathrm{C}(50) & 174.32(18) \\ \mathrm{Ru}(1)-\mathrm{O}(4)-\mathrm{C}(34)-\mathrm{C}(50) & -38.2(2) \\ \mathrm{C}(33)-\mathrm{C}(15)-\mathrm{C}(35)-\mathrm{C}(23) & -0.5(4) \\ \mathrm{C}(24)-\mathrm{C}(23)-\mathrm{C}(35)-\mathrm{C}(15) & 179.2(2) \\ \mathrm{C}(9)-\mathrm{C}(23)-\mathrm{C}(35)-\mathrm{C}(15) & -0.5(4) \\ \mathrm{C}(16)-\mathrm{C}(28)-\mathrm{C}(36)-\mathrm{C}(41) & -3.1(4) \\ \mathrm{N}(2)-\mathrm{C}(28)-\mathrm{C}(36)-\mathrm{C}(41) & -179.6(2) \\ \mathrm{C}(16)-\mathrm{C}(28)-\mathrm{C}(36)-\mathrm{C}(48) & 179.3(3) \\ \mathrm{N}(2)-\mathrm{C}(28)-\mathrm{C}(36)-\mathrm{C}(48) & 2.7(4) \\ \mathrm{C}(32)-\mathrm{C}(13)-\mathrm{C}(38)-\mathrm{C}(39) & 3.2(3) \\ \mathrm{C}(30)-\mathrm{C}(13)-\mathrm{C}(38)-\mathrm{C}(39) & -175.9(2) \\ \mathrm{C}(13)-\mathrm{C}(38)-\mathrm{C}(39)-\mathrm{C}(44) & -2.0(4) \\ \mathrm{C}(13)-\mathrm{C}(32)-\mathrm{C}(40)-\mathrm{C}(44) & 0.4(4) \\ \mathrm{C}(45)-\mathrm{C}(27)-\mathrm{C}(41)-\mathrm{C}(36) & 0.4(4) \\ \mathrm{C}(49)-\mathrm{C}(27)-\mathrm{C}(41)-\mathrm{C}(36) & -179.3(3) \\ \mathrm{C}(28)-\mathrm{C}(36)-\mathrm{C}(41)-\mathrm{C}(27) & 1.0(4) \\ \mathrm{C}(48)-\mathrm{C}(36)-\mathrm{C}(41)-\mathrm{C}(27) & 178.8(3) \\ \mathrm{C}(22)-\mathrm{C}(20)-\mathrm{C}(42)-\mathrm{C}(43) & -4.4(4) \\ \mathrm{C}(20)-\mathrm{C}(42)-\mathrm{C}(43)-\mathrm{C}(30) & 2.2(4) \\ \mathrm{C}(14)-\mathrm{C}(30)-\mathrm{C}(43)-\mathrm{C}(42) & 3.6(4) \\ \mathrm{C}(13)-\mathrm{C}(30)-\mathrm{C}(43)-\mathrm{C}(42) & -171.6(2) \\ \mathrm{C}(32)-\mathrm{C}(40)-\mathrm{C}(44)-\mathrm{C}(39) & 0.9(5) \\ \mathrm{C}(38)-\mathrm{C}(39)-\mathrm{C}(44)-\mathrm{C}(40) & \\ \mathrm{C}(41)-\mathrm{C}(27)-\mathrm{C}(45)-\mathrm{C}(16) & \\ & \\ & \\ & \end{array}$




\begin{tabular}{|c|c|}
\hline $\mathrm{C}(49)-\mathrm{C}(27)-\mathrm{C}(45)-\mathrm{C}(16)$ & $179.8(3)$ \\
\hline $\mathrm{C}(28)-\mathrm{C}(16)-\mathrm{C}(45)-\mathrm{C}(27)$ & $-2.1(4)$ \\
\hline $\mathrm{C}(47)-\mathrm{C}(16)-\mathrm{C}(45)-\mathrm{C}(27)$ & $179.4(3)$ \\
\hline $\mathrm{C}(8)-\mathrm{C}(29)-\mathrm{O}(1)-\mathrm{Ru}(1)$ & $-70.7(3)$ \\
\hline $\mathrm{C}(5)-\mathrm{C}(29)-\mathrm{O}(1)-\mathrm{Ru}(1)$ & $115.3(2)$ \\
\hline $\mathrm{C}(54)-\mathrm{Ru}(1)-\mathrm{O}(1)-\mathrm{C}(29)$ & $-97.75(17)$ \\
\hline $\mathrm{C}(55)-\mathrm{Ru}(1)-\mathrm{O}(1)-\mathrm{C}(29)$ & $4.92(17)$ \\
\hline $\mathrm{O}(4)-\mathrm{Ru}(1)-\mathrm{O}(1)-\mathrm{C}(29)$ & $-176.63(16)$ \\
\hline $\mathrm{I}(2)-\mathrm{Ru}(1)-\mathrm{O}(1)-\mathrm{C}(29)$ & 109.06(16) \\
\hline $\mathrm{C}(10)-\mathrm{C}(6)-\mathrm{N}(1)-\mathrm{C}(55)$ & $-80.4(3)$ \\
\hline $\mathrm{C}(21)-\mathrm{C}(6)-\mathrm{N}(1)-\mathrm{C}(55)$ & $101.4(3)$ \\
\hline$C(10)-C(6)-N(1)-C(52)$ & $83.5(3)$ \\
\hline $\mathrm{C}(21)-\mathrm{C}(6)-\mathrm{N}(1)-\mathrm{C}(52)$ & $-94.7(3)$ \\
\hline $\mathrm{C}(28)-\mathrm{N}(2)-\mathrm{C}(55)-\mathrm{N}(1)$ & $-176.4(2)$ \\
\hline $\mathrm{C}(51)-\mathrm{N}(2)-\mathrm{C}(55)-\mathrm{N}(1)$ & $2.5(3)$ \\
\hline $\mathrm{C}(28)-\mathrm{N}(2)-\mathrm{C}(55)-\mathrm{Ru}(1)$ & $6.0(4)$ \\
\hline $\mathrm{C}(51)-\mathrm{N}(2)-\mathrm{C}(55)-\mathrm{Ru}(1)$ & $-175.1(2)$ \\
\hline $\mathrm{C}(6)-\mathrm{N}(1)-\mathrm{C}(55)-\mathrm{N}(2)$ & $173.4(2)$ \\
\hline $\mathrm{C}(52)-\mathrm{N}(1)-\mathrm{C}(55)-\mathrm{N}(2)$ & $8.4(3)$ \\
\hline $\mathrm{C}(6)-\mathrm{N}(1)-\mathrm{C}(55)-\mathrm{Ru}(1)$ & $-8.4(3)$ \\
\hline $\mathrm{C}(52)-\mathrm{N}(1)-\mathrm{C}(55)-\mathrm{Ru}(1)$ & $-173.41(18)$ \\
\hline $\mathrm{C}(54)-\mathrm{Ru}(1)-\mathrm{C}(55)-\mathrm{N}(2)$ & $-4.9(3)$ \\
\hline $\mathrm{O}(1)-\mathrm{Ru}(1)-\mathrm{C}(55)-\mathrm{N}(2)$ & $-112.9(2)$ \\
\hline $\mathrm{O}(4)-\mathrm{Ru}(1)-\mathrm{C}(55)-\mathrm{N}(2)$ & $96.6(12)$ \\
\hline $\mathrm{I}(2)-\mathrm{Ru}(1)-\mathrm{C}(55)-\mathrm{N}(2)$ & $96.6(2)$ \\
\hline $\mathrm{C}(54)-\mathrm{Ru}(1)-\mathrm{C}(55)-\mathrm{N}(1)$ & 177.61(17) \\
\hline $\mathrm{O}(1)-\mathrm{Ru}(1)-\mathrm{C}(55)-\mathrm{N}(1)$ & $69.63(17)$ \\
\hline $\mathrm{O}(4)-\mathrm{Ru}(1)-\mathrm{C}(55)-\mathrm{N}(1)$ & $-80.9(13)$ \\
\hline $\mathrm{I}(2)-\mathrm{Ru}(1)-\mathrm{C}(55)-\mathrm{N}(1)$ & $-80.84(16)$ \\
\hline $\mathrm{C}(55)-\mathrm{N}(1)-\mathrm{C}(52)-\mathrm{C}(51)$ & $-14.9(3)$ \\
\hline $\mathrm{C}(6)-\mathrm{N}(1)-\mathrm{C}(52)-\mathrm{C}(51)$ & $179.7(2)$ \\
\hline $\mathrm{C}(55)-\mathrm{N}(2)-\mathrm{C}(51)-\mathrm{C}(52)$ & $-11.4(3)$ \\
\hline $\mathrm{C}(28)-\mathrm{N}(2)-\mathrm{C}(51)-\mathrm{C}(52)$ & $167.6(2)$ \\
\hline $\mathrm{N}(1)-\mathrm{C}(52)-\mathrm{C}(51)-\mathrm{N}(2)$ & $14.6(3)$ \\
\hline
\end{tabular}

